DELLINGER, ANTHONY, Ph.D. Fullerenes and their Potential in Nanomedicine. (2015)

Directed by Dr. Christopher L. Kepley. 155 pp.

Carboxyfullerenes are closed carbon spheres which are being actively pursued globally for a wide range of applications. Their inherent properties and ability to be functionalized with side-chains results in nearly limitless new chemical structures making them ideal platform molecules for new solutions to basic biological problems. In general, two classes of fullerenes exist for nanomedicine applications; empty cage and metallofullerenes. The carbon cage (usually $\mathrm{C}_{60}$ and $\mathrm{C}_{70}$ ) of empty cage fullerenes are antioxidants, thus potential therapeutics for inflammatory diseases. Our discovery that certain fullerene derivatives can stabilize and prevent pro-inflammatory mediator release from human tissue mast cells make them ideal candidates for diseases controlled by MC mediators (e.g. arthritis, asthma, etc.). New research findings using metallo-fullerenes with gadolinium inside the carbon cage as diagnostics using Magnetic Resonance Imaging for inflammatory diseases suggest metallo-fullerenes are more sensitive than current contrast agents, have the ability to be targeted to disease specific biomarkers, and are safe. 


\title{
FULLERENES AND THEIR POTENTIAL IN NANOMEDICINE
}

\author{
by
}

\begin{abstract}
Anthony Dellinger
\end{abstract}

\author{
A Dissertation Submitted to \\ the Faculty of the Graduate School at \\ The University of North Carolina at Greensboro \\ in Partial Fulfillment \\ of the Requirements for the Degree \\ Doctor of Philosophy
}

\section{Greensboro}

2015

Approved by

Committee Chair 


\title{
APPROVAL PAGE
}

This dissertation has been approved by the following committee of the Faculty of the Graduate School at the University of North Carolina at Greensboro.

\author{
Committee Chair
}

Committee Members

Date of Acceptance by Committee

Date of Final Oral Examination 


\section{ACKNOWLEDGEMENTS}

Thanks to all who endured. 


\section{CHAPTER}

I.1 Fullerenes as a Platform for New Solutions in

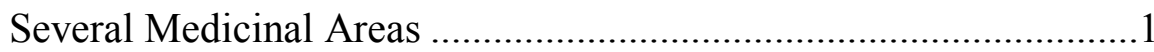

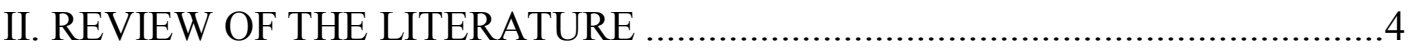

II.1 Fullerenes as Therapeutics ................................................................

II.2 Fullerenes as Carriers for Drug and Gene Delivery ................................5

II.3 Ability of Fullerenes to Affect Mast

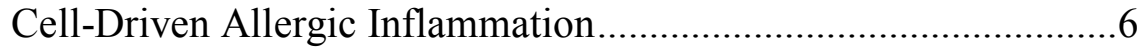

II.4 Ability of Fullerenes to Dampen Asthma Pathogenesis..........................

II.5 Fullerenes Inhibit Inflammation Caused by Arthritis ...........................12

II.6 Fullerenes as a Potential Therapy for MS …………………..............16

II.7 Fullerenes as Diagnostics ...................................................................20

II.8 Endo-Fullerene Contrast Agents as Diagnostics for Atherosclerotic Plaque ...........................................................22

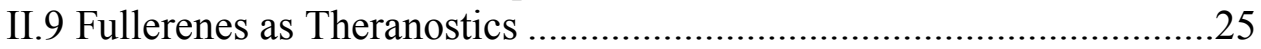

II.10 Endo-Fullerene Contrast Agents as Theranostics

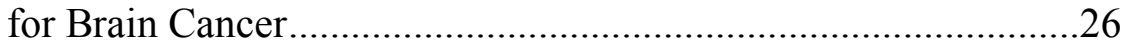

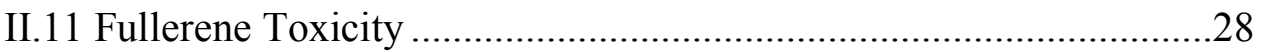

\section{FULLERENES INHIBIT ALLERGIC MEDIATOR RELEASE} FROM HUMAN MAST CELLS AND BASOPHILS ...................................33

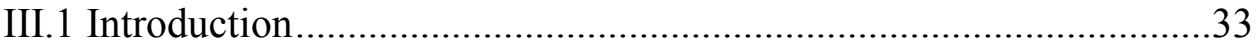

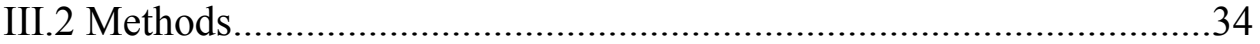

III.2.1 Fullerene derivatives .........................................................

III.2.2 Human mast cell and peripheral blood basophil cultures and activation........................................35

III.2.3 Reactive oxygen species and calcium measurements..........36

III.2.4 Immunoblotting analysis of signal transduction intermediates ……............................................................ 
III.3 Results.

III.3.1 Fullerenes differentially effect mast cell non-IgE-induced mediator release

III.3.2 The effect of FD on non-IgE induced mast cell PGD2 release

III.3.3 The effect of FD on non-IgE induced peripheral blood basophil mediator release

III.3.4 The effect of FD on non-IgE mediated induced ROS activity and intracellular calcium flux in mast cell

III.3.5 The effect of FD on non-IgE mediated signaling pathway intermediates

III.4 Conclusions

IV. FUNCTIONALIZED GADOLINIUM ENDOFULLERENES

DETECT ATHEROSCLERTOIC PLAQUE LESIONS .52

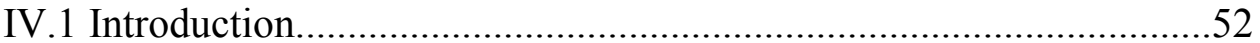

IV.2 Methods .53

IV.2.1 ATCA synthesis, functionalization, and characterization 53

IV.2.2 Cell culture .54

IV.2.3 Cell uptake of the ATCA revealed by FIB-SEM crossbeam microscopy.

IV.2.4 Western blotting and quantification of CD36-specific phosphor-signaling intermediates .55

IV.2.5 Flow cytometry..... .56

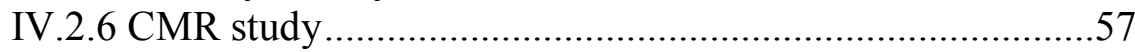

IV.2.7 Signal enhancement measurement........................................57

IV.2.8 Histology, immunohistochemistry, and Gd

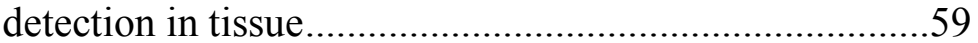

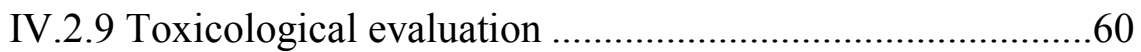

IV.3 Results.

IV.3.1 Plaque targeting contrast agents are selectively taken up by foam cells through CD36 receptor ................60

IV.3.2 ATCA detect atherosclerotic plaque in vivo .66

IV.3.3 Tracking the in vivo fate and toxicity of plaque-targeting contrast agents

IV.4 Conclusions .68

V. A STEROID-MIMICKING FULLERENE THAT MEDIATES INHIBITION OF HUMAN LUNG MAST CELL RESPONES. 


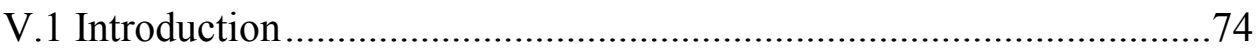

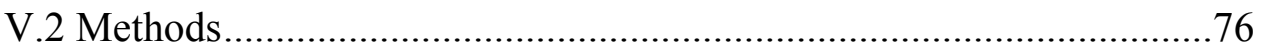

V.2.1 Fullerenes synthesis and characterization .............................76

V.2.2 Gene microarray................................................................

V.2.3 Immuno-blotting and immuno-precipitation...........................77

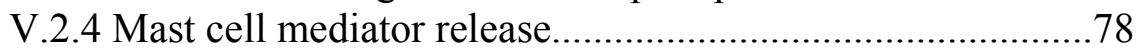

V.3 Results

V.3.1 Pretreatment of mast cells with fullerenes upregulates DUSP1 gene and protein levels .....................79

V.3.2 Fullerene upregulation of DUSP1 protein expression modulates activation of MAPK ……………....81

V.3.3 Fullerenes and corticosteroids inhibit FCeRI-mediated-MC degranulation ..................................83

V.3.4 Co-incubations of fullerenes or corticosteroids with long-acting beta2-adrenergic receptor agonists inhibits FCeRI-mediated-MC

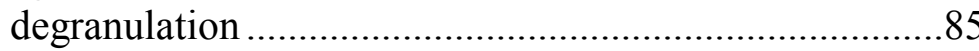

V.3.5 Fullerenes and corticosteroids inhibit FCeRimediated-MC GM-CSF cytokine production ...................87

V.4 Conclusions..... . .90

\section{INHIBITION OF INFLAMMATORY ARTHRITIS USING}

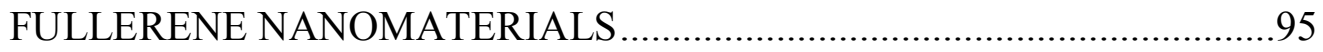

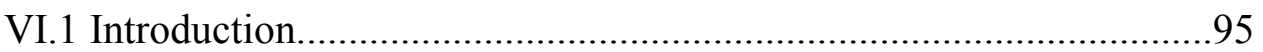

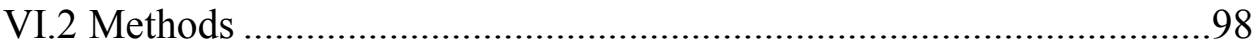

VI.2.1 Fullerene derivatives........................................................98

VI.2.2 Inflammatory mediator release from BMMC

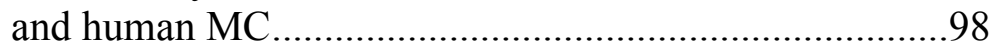

VI.2.3 Synovial fibroblast cytokine production................................99

VI.2.4 Osteoclast differentiation.....................................................99

VI.2.5 Measuring NF- $\mathrm{kB}$ levels using in-cell Western..................100

VI.2.6 Mitochondrial membrane potential.....................................101

VI.2.7 Reactive oxygen species measurements ............................102

VI.2.8 Odyssey imaging of fullerene derivatives in vivo .............103

VI.2.9 Inflammatory arthritis ....................................................103

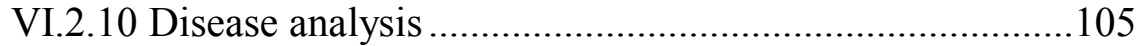

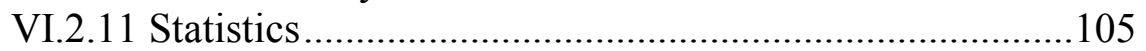

VI.3 Results. 106

VI.3.1 The efficacy of fullerene derivative inhibition on inflammatory mediator release and osteoclast formation depends on functional moieties added to the carbon cage 
VI.3.2 Fullerene derivatives inhibit mitochondrial membrane potential, ROS production, and $\mathrm{NF}-\mathrm{\kappa B}$ activation.

VI.3.3 ALM targets the inflamed synovial joints, but not organs, in vivo. ................................................111

VI.3.4 ALM and TGA prevent inflammatory arthritis. ..................114

VI.3.5 Toxicological assessment of ALM and TGA.

VI.4 Conclusions 118

VII. FUTURE PERSPECTIVES

REFERENCES 126

APPENDIX A. DESIGN SYNTHESIS OF ATCA.

APPENDIX B. DLS, ZETA POTENTIAL, AND RELAXIVITY OF LIPOSOME CONTROL AND ATCA 


\section{LIST OF TABLES}

Page

Table 3.1. Mean \% Inhibition of Degranulation $( \pm \mathrm{SD})$ in FD Treated and Untreated MC. 38

Table 3.2. Mean \% Inhibition of TNF- $\alpha( \pm \mathrm{SD})$ in FD Treated and Untreated MC.

Table 3.3. Mean \% Inhibition of PGD2 Production $( \pm \mathrm{SD})$ in FD Treated and Untreated $\mathrm{MC}$

Table 3.4. Mean \% Inhibition of Degranulation $( \pm \mathrm{SD})$ in FD Treated and Untreated PBB.

Table 3.5. Mean \% Inhibition of IL-13 $( \pm \mathrm{SD})$ in FD Treated and Untreated PBB.

Table 4.1. Total Percent Gadolinium in Tissue. 67

Table 5.1. Mean \% FceRI-Mediated Degranulation in $\mathrm{C}_{70}$-I or ICS (Fluticasone or Budesonide) Treated and Untreated Lung MC.

Table 5.2. Synergistic Effects on \% FceRI-Mediated Degranulation in Fullerene $\left(\mathrm{C}_{70} \mathrm{I}\right)$ and LABA (Salmeterol) or ICS (Fluticasone or Budesonide) and LABA (Salmeterol) Treated and Untreated MC. 


\section{LIST OF FIGURES}

Page

Figure 1.1. Representative Fullerene Structures.................................................. 2

Figure 2.1. Characterization of the Novel Fullerene Derivative

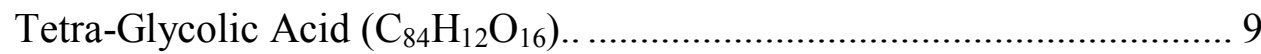

Figure 2.2. Fullerenes Reduce Asthma Airway Hyper-Responsiveness. ...................... 10

Figure 2.3. Fullerenes Reduce Airway Inflammation by Upregulation of Eicosanoids in Bronchoalveolar Lavage Fluid of Mice.

Figure 2.4. Characterization of the Novel Fullerene Derivative Amphiphilic

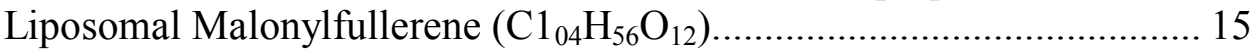

Figure 2.5. Fullerenes Attenuate Inflammatory Arthritis of the $\mathrm{K} / \mathrm{BxN}$-Induced Disease Pathology 16

Figure 2.6. A Neuron-Targeting Fullerene Crosses the Blood-Brain Barrier. 18

Figure 2.7. Fullerene-Tropane Derivatives can Target the Neuronal Dopamine Transporter Receptors on Human Neuroblastoma Cells 19

Figure 2.8. Fullerene Derivatives Inhibit the Onset of Experimental Allergic Encephalomyelitis

Figure 2.9. Basic Structure of Atherosclerosis-Targeting Contrast Agents.

Figure 2.10. Atherosclerotic Plaque-Targeting Contrast Agents can Detect Plaque In Vivo.

Figure 2.11. Glioblastoma-Targeting Theranostic

Figure 2.12. Glioblastoma-Targeting Nanovesicles with a Therapeutic Payload

Attached can Shrink Brain Tumors.

Figure 3.1. Specific FD Inhibit Human MC Calcium-Ionophore and Poly-LLysine-Induced Mediator Release in a Dose Dependent Manner 
Figure 3.2. Specific FD Inhibit Human MC Compound 48/80- and Somatostatin-Induced Degranulation in a Dose Dependent Manner

Figure 3.3. Fullerene Derivatives Inhibit Secretagogue-Induced Elevations in Intracellular ROS Levels

Figure 3.4. Fullerene Derivatives Inhibit Secretagogue-Induced Elevations in Intracellular Calcium Levels

Figure 3.5. Inhibition of Mediator Release in Calcium Ionophore, Compound 48/80, Poly-L-Lysine, and Somatostatin Challenged MC Involves the Down-Regulation of MAPK and LAT Phosphorylation.

Figure 4.1. ATCA can Detect Inflammatory Plaque In Vivo......................................... 58

Figure 4.2. Upregulation of CD36 Receptors on Foam Cells..................................... 61

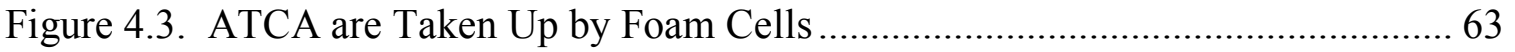

Figure 4.4. ATCA Activate CD36-Specific Signaling Intermediates.............................. 65

Figure 4.5. Plaque-Targeting Contrast Agents do not Accumulate in Major Organs

Figure 5.1. Mast Cell Pretreatment with $\mathrm{C}_{70}$-I Increases DUSP1 Gene and DUSP1 Protein Expression.

Figure 5.2. Mast Cell Pretreatment with $\mathrm{C}_{70}$ I Decreases Phosphorylation of MAPK1

Figure 5.3. The Effects of MC Pretreatment with $\mathrm{C}_{70} \mathrm{I} / \mathrm{ICS}$ Individually or in Combination with LABA on Degranulation.

Figure 5.4. The Effects of MC Pretreatment with $\mathrm{C}_{70}-\mathrm{I} / \mathrm{ICS}$ Individually or in

Combination with LABA on GM-CSF Cytokine Production. 89

Figure 6.1. Fullerene Derivatives Reduce Degranulation and Cytokine Production from Synovial Fibroblast from RA Patients, Mouse BMMC, and Human MC (hMC), and Osteoclast Formation from Human PBMC.. 108 
Figure 6.2. Mitochondrial Membrane Potential Correlates with MC Degranulation Through FceR Receptors and is Inhibited by Fullerene Derivatives ....................................................................... 110

Figure 6.3. ALM Targets Joints in Inflammatory Arthritis ........................................... 113

Figure 6.4. Fullerene Derivatives Attenuate Inflammatory Arthritis in the $\mathrm{K} / \mathrm{BxN}$ but not CIA Model. .............................................................................. 116

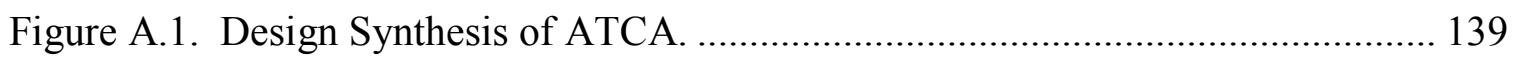

Figure B.1. DLS, Zeta Potential, and Relaxivity of Liposome Control and ATCA...... 142 


\section{CHAPTER I}

\section{INTRODUCTION}

Fullerenes are carbon spheres presently being pursued globally for a wide range

of applications in nanomedicine. These molecules have unique electronic properties that make them attractive candidates for diagnostic, therapeutic and theranostic applications. Herein, the latest research is discussed on developing fullerene-based therapeutics as antioxidants for inflammatory diseases and the promise of endohedral fullerenes as new MRI contrast agents. The recent discovery that certain fullerene derivatives can stabilize immune effector cells to prevent or inhibit the release of proinflammatory mediators makes them potential candidates for several diseases such as asthma, arthritis, and multiple sclerosis. Gadolinium-containing endohedral fullerenes are being pursued as diagnostic MRI contrast agents for several diseases.

\section{I.1 Fullerenes as a Platform for New Solutions in Several Medicinal Areas}

Empty cage fullerenes (Figure 1.1A \& B) have unique electrochemical properties and have a wide range of potentially beneficial biologic properties. Another type of fullerene can have metals enclosed inside them (Figure 1.1C). Fullerenes have a unique cage structure with delocalized p-molecular orbital electrons. This structure confers unusual activity in electron transfer systems due to their low reorganization energy, low lying excited states (singlet and triplet) and extended triplet lifetimes. Furthermore, the 
spherical configuration of the planar benzene rings imposes an unusual constraint on these p-electron orbitals. The native fullerene cage is insoluble in water and must be derivatized (simply meaning moieties or side groups must be added to the carbon cage) in order to make them water soluble (compatible in biological systems). The ability of fullerenes to be derivatized with side chains provides opportunities to diversify, manipulate, and harness the electronic properties of the cage for selected applications. Of course, each derivation results in changes of the compound's physical and chemical properties, including particle size/length, z-potential, molecular weight, surface characteristics and solubility, contributing to how they affect biological systems. All too often, results from studies examining the effects of fullerenes on biological systems tend to be extrapolated to other applications.

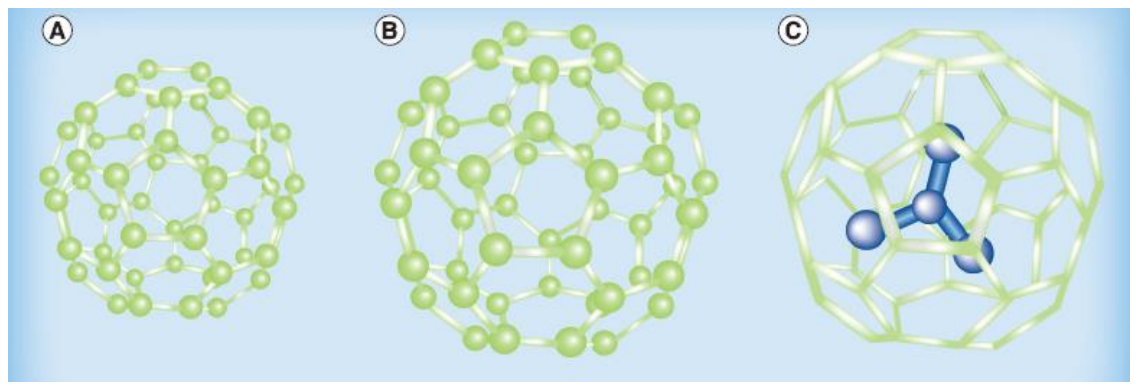

Figure 1.1. Representative Fullerene Structures. (A) Empty cage $C_{60}$ fullerene, (B) empty cage $C_{70}$ fullerene and $(C)$ gadolinium-enclosed $C_{80}$ endohedral fullerene.

As demonstrated below, each fullerene derivative (FD) must be assessed separately depending on the biological application. Even extremely similar FDs can have completely opposite results, which stimulates efforts to understand how changing the chemical composition and structural arrangement of fullerenes affects molecular 
interactions at the cellular, tissue and organ system levels. Consequently, their inherent properties described in the article, combined with their ability to be derivatized with side chains, results in almost limitless new chemical structures, making them ideal platform molecules for innovative new solutions to basic biological problems. Fullerenes are one of many types of carbon nanomaterials being investigated for next-generation medical applications; presently carbon nanotubes are being investigated as drug carriers (manipulating their hollow internal core), biosensensors (attaching enzymes to their walls) and targeted transporters of drugs [1-4]. The 2D structure graphene is being investigated as a drug/gene delivery nanocarrier and targeted cancer therapy $[5,6]$. This thesis will focus on the applications of both empty cage derivatized and endohedral fullerenes for therapeutic, diagnostic and theranostic (combination of diagnostic and therapeutic) applications. 


\section{CHAPTER II}

\section{REVIEW OF THE LITERATURE}

\section{II.1 Fullerenes as Therapeutics}

Fullerenes have been coined 'free radical sponges' and described most frequently as antioxidants, although in biological systems they can paradoxically act as both oxidants and antioxidants. The generation of free radicals such as reactive oxygen species (ROS) and reactive nitrogen species occurs naturally in cells, and their presence at sites of disease pathologies suggests they contribute to disease progression. The term free radical refers to a molecular species that possesses an unpaired electron, which makes them highly reactive. Many of the more common ROS or reactive nitrogen species that contribute to oxidative or nitrosative stress in biological systems are free radicals, including hydroxyl radicals $(\mathrm{OH} \bullet)$, superoxide anions $\left(\mathrm{O}^{-}\right)$, and peroxynitrites $\left(\mathrm{ONOO}^{-}\right)$. Other ROS are not free radicals, including singlet oxygen and hydrogen peroxide $\left(\mathrm{H}_{2} \mathrm{O}_{2}\right)$, which are considered ROS because they can generate oxygen radicals, such as superoxide, via the Fenton reaction. These ROS can react with, crosslink and alter the function of many macromolecules. These species can negatively affect a wide variety of biological processes; however, in certain situations they can be beneficial to some biological processes such as signaling and cellular defenses. Antioxidants (e.g., vitamins A and C) are molecules that can eliminate or neutralize free radical electrons. This has led to a tremendous amount of research on preventing damage using antioxidants, 
theorized to, in turn, counteract disease pathologies. Indeed the perceived benefits of antioxidants are a widely accepted concept yet their use has been mainly centered on over-the-counter supplements for general health and antiaging benefits.

The carbon cage (usually $\mathrm{C}_{60}$ and $\mathrm{C}_{70}$ ) of empty cage fullerenes (Figure 1.1) can have antioxidant functionality based on its ability to absorb electrons and disperse them through the 3D $\pi$-conjugated structure distributed over its surface. This ability to scavenge free radicals has led to their potential as a new way for treating a wide range clerosis (MS) [7], neurodegenerative [8], anti-HIV activity [9, 10], cancer [11], radiation exposure [12], ischemia [13], osteoporosis [14], general inflammation [15] and selective antimicrobial agents against bacteria [16]. Interestingly, mice and rats chronically treated with water-solubilized carboxylated fullerenes have significantly extended lifespans compared with littermate controls $[17,18]$. There are almost endless possibilities for adding side groups to the carbon cage to induce functionality, resulting in potential answers to some of modern medicine's most elusive problems.

\section{II.2 Fullerenes as Carriers for Drug and Gene Delivery}

Recently, much interest and focus has been directed towards developing safe, targeted and competent transport mechanisms for drugs and genes to cells. This transport is often riddled with difficulties as the cellular membrane creates efficient barriers that can prevent or slow the uptake of compounds. However, the small size $(<5 \mathrm{~nm})$ of fullerenes has generated significant interest in the field. Hydrophillic entities can be decorated around the fullerene cage, modifying the fullerenes relative insolubility in 
aqueous solutions. The water-soluble FD can readily cross the cellular membrane without damaging cells $[8,19]$. These fullerenes can be further modified to become carriers of drugs and genes. Evaluated below is some of the present research in which fullerenes have been functionalized to become water soluble and utilized as versatile nanocarriers to target, treat and prevent disease with high biocompatibility.

\section{II.3 Ability of Fullerenes to Affect Mast Cell-Driven Allergic Inflammation}

Allergic reactions are the result of B-cell-produced, specific IgE antibody to common, normally innocuous antigens. In simplistic terms, mast cells (MCs), peripheral blood basophils (PBBs), natural killer cells, T cells and even B cells are responsible for driving the initial allergen-inducing reaction through the production of IL-4 and other Th2-specific cytokines, which result in IgE sensitization. Re-exposure to the allergen triggers an allergic response through the release of inflammatory mediators from MCs and PBBs. The IgE produced binds to FceRI on MCs and PBBs, and the release of preallergic mediators is induced when two or more IgE molecules are crosslinked with the allergen. Indeed, most allergy medications are aimed at neutralizing (antihistamines and $\mathrm{H} 1$-receptor blockers) or preventing (omalizumab) $\mathrm{MC}$ and PBB FceRI responses. New research has demonstrated that specifically engineered FDs are taken up and can stabilize human tissue MCs, preventing the release of proinflammatory mediators from these cells, making them ideal candidates for those diseases controlled by MC mediators [20-22]. The ability for FDs to stabilize MC-driven diseases in vitro should translate to blocking in vivo, as shown by Dellinger et al. [21]. 


\section{II.4 Ability of Fullerenes to Dampen Asthma Pathogenesis}

MCs are ubiquitously expressed in tissues and are uniquely able to initiate and propagate certain inflammatory responses, as well as offer an interface between innate and adaptive immunity [23]. Mice without MCs fail to develop asthma-like pulmonary disease when sensitized with less aggressive immunization protocols and challenged with aerosolized allergens $[24,25]$. However, the strongest evidence that MCs are critical for human asthma comes from the many therapeutics used to treat the disease in humans. In general, two categories of therapies were developed to control asthma; blocking MC activation before it occurs (stabilizers) and blocking MC activation after it occurs. MCs are the only cells in the lung that have prestored TNF- $\alpha$ in their granules that can be immediately released upon allergen provocation [26] and blockade of this cytokine is a valid target currently being investigated [27]. Therapies that block the effects of MCs after activation occurs are well known, commonly used, and include antihistamines and H1-receptor blockers. Thus, the importance of MCs in asthma pathogenesis is well established, and controlling the amount of mediator release from these cells is a proven drug development strategy.

The role of basophils in asthma is less clear. Their role in allergic disease has largely been viewed as redundant to that of tissue MCs. This line of thought, however, is changing with evidence that has emerged during the last 15 years. These cells are a significant source of the cytokines IL-4 and IL-13, both of which are vital to the pathogenesis of allergic disease, including asthma. It has been demonstrated that increased levels of basophils were present in the lungs of deceased asthmatics using a 
basophil-specific antibody developed for immunologically detecting these cell types [28, 29]. Thus, the infiltration of basophils into allergic lesion sites has sparked greater interest in this once overlooked immune cell, both in adaptive as well as innate immunity [30].

There is a strong need for novel therapeutics to treat asthmatic disease; indeed up to $55 \%$ of patients receiving treatment for asthma have uncontrolled symptomology [31]. In some situations, especially in less severe asthmatics, asthma control is achieved with the use of nonspecific anti-inflammatories, such as corticosteroids, or by the use of antihistamines and leukotriene inhibitors. However, these therapies are generally less effective in severe asthmatics or in patients with steroid resistance. As shown below, FDs are able to suppress both disease onset and reverse established disease in murine asthma models. The latter is especially important as human asthma treatment always involves established disease.

The utility of FD's role in asthma prevention and reversal can be validated in mice treated with FDs throughout ovalbumin challenge. Treated animals have significantly less airway inflammation and bronchoconstriction compared with untreated animals. In fact, total inflammation and bronchoconstriction in the tetraglycolic acid FDtreated animals are not only significantly reduced, but are similar to that seen in nonsensitized controls (Figure 2.1) [32]. Thus, symptoms of disease are largely reversed in these animals. These studies used a model previously shown to utilize MCs [25]. In the established disease model, where mice were treated throughout disease development, FDs dampened eosinophilia and cytokine levels significantly in the bronchoalveolar lavage 
(BAL) fluid. Lung sections show massive cellular infiltration in untreated animals, while those receiving FDs have minimal cellular infiltration surrounding the airways (Figure 2.2). This led to reduced airway hyper-responsiveness in FD-treated animals. Thus, FDs may be useful in a clinical setting to reverse asthma pathogenesis and limit exacerbation of symptoms.

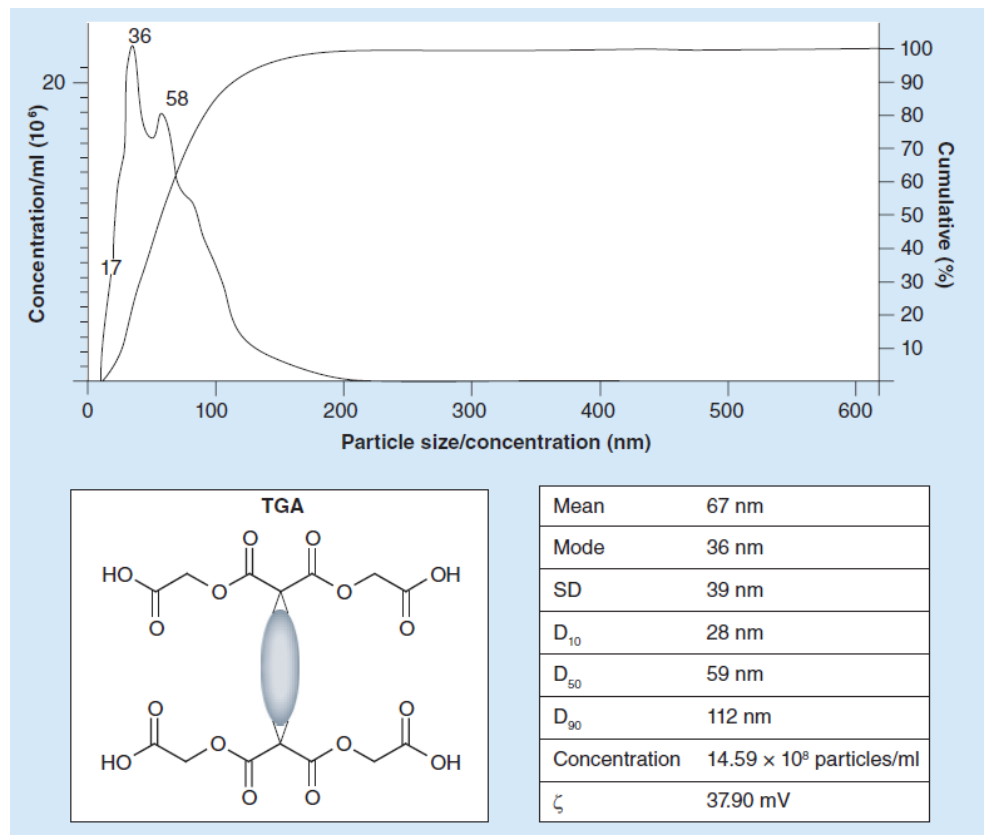

Figure 2.1. Characterization of the Novel Fullerene Derivative Tetra-Glycolic Acid $\left(\mathrm{C}_{84} \mathrm{H}_{12} \mathrm{O}_{16}\right)$. $\mathrm{D}_{10}$ : Diameter where $10 \%$ of the population have diameters below that size; $D_{50}$ : Median diameter where half of the population have diameters below that size; $D_{90}$ : Diameter where $90 \%$ of the diameters are below that size; SD: Standard deviation; TGA: Tetra-glycolic acid. 


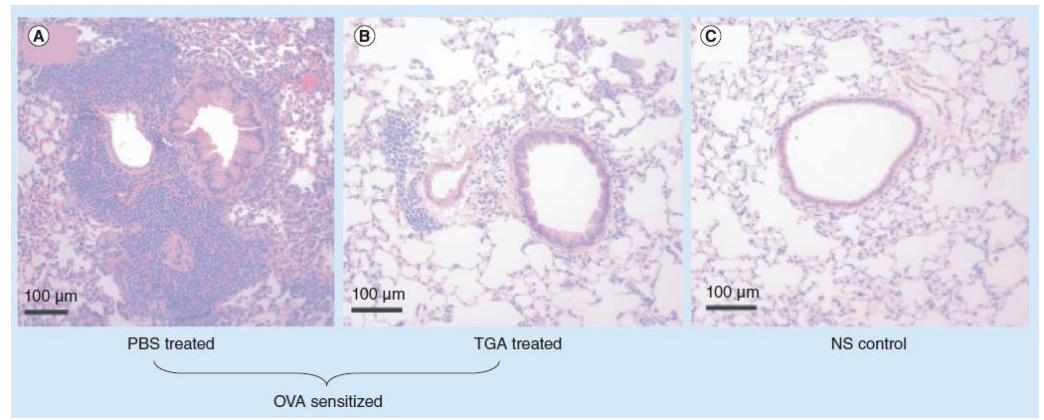

Figure 2.2. Fullerenes Reduce Asthma Airway Hyper-Responsiveness. (A) Asthmatic inflammation induced in the lung is inhibited in (B) fullerene derivativetreated mice compared with $(C)$ NS controls. NS: Nonsensitized; OVA: Ovalbumin; PBS: Phosphate-buffered saline; TGA: Tetra-glycolic acid.

While previously published in vitro studies suggested that MC inhibition may be the predominant mechanism of FD inhibition [20, 22], in vivo studies suggest multipotent effects of these unique compounds. FD treatment reduces the levels of BAL Th2 proinflammatory cytokines and reduces lung inflammation. While IL-4 stimulates IgE production by B cells, IL-5 both recruits and activates eosinophils at the site of inflammation. FD treatment significantly reduces both IL-4 and IL-5 in the BAL fluid. Additionally, serum IgE levels were significantly reduced following FD treatment.

Importantly, the use of FD revealed a novel mechanism of action and opened up new avenues of research for asthma. Several eicosanoids (EETs) derived from the CYP450 pathway are relatively stable, and these molecules were measured in BAL fluid samples using mass spectrometry. The EETs are consistently associated with relaxation of the bronchi and other in vivo anti-inflammatory actions $[33,34]$. The epoxyeicosatrienoic acid was consistently upregulated in BAL fluid from FD-treated mice (Figure 2.3). Further in vivo studies demonstrated that the EETs play a major role in dampening the 
asthma phenotype as inhibitors of the EETs prevented the FD-induced modulation of the ovalbumin-induced asthma model [32]. Certain EETs stabilize human lung MCs through the inhibition of FceRI-mediator release and upregulate the $C Y P 1 B 1$ gene in these cells (Figure 2.3). Thought to be produced by lung epithelial and endothelial cells, the EETs have been shown to relax histamine-precontracted guinea pig and human bronchi [35, 36]. Furthermore, they can inhibit the upregulation of VCAM-1, E-selectin, and ICAM-1, thus, potentially limiting cellular infiltration of the lung [37]. Consequently, epoxyeicosatrienoic acid upregulation plays a significant role in dampening airway inflammation and bronchoconstriction in these models. FD upregulation of EETs is a novel mechanism for controlling asthma and suggests that strategies that induce the production of EETs may be a viable therapeutic strategy for treating asthmatics.

The efficacy of FD for the treatment of asthma is illustrated through a mechanism involving the dampening of eosinophilia and cytokine levels, reducing airway hyperresponsiveness and upregulating EETs. Thus, FD compounds have the potential to become novel therapeutics for the treatment of asthma and pave the way for new research efforts focusing on the role of EETs in human disease. 

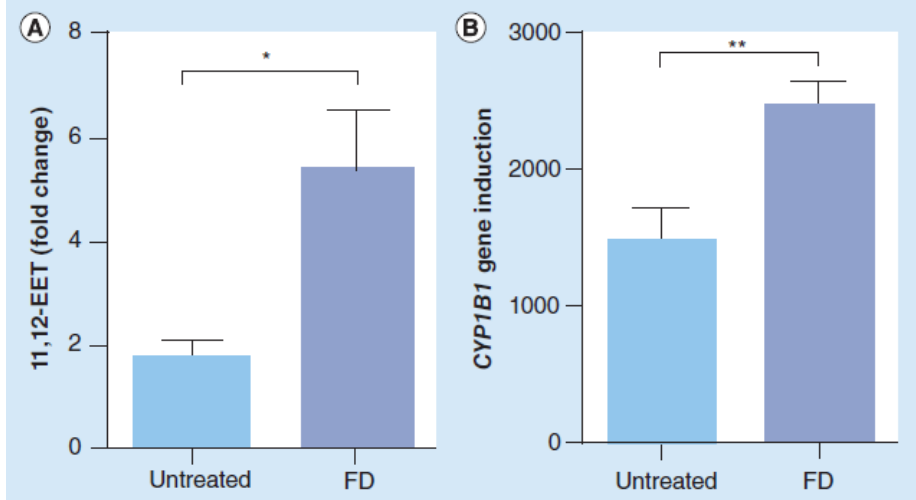

Figure 2.3. Fullerenes Reduce Airway Inflammation by Upregulation of Eicosanoids in Bronchoalveolar Lavage Fluid of Mice. (A) FDs upregulate EETs in branchoalveolar lavage fluid compared with untreated controls and $(B)$ induce CYP1B1 gene inducton. *Statistical significance $(p<0.05)$. EET: Eicosanoid; FD: Fullerene derivative.

\section{II.5 Fullerenes Inhibit Inflammation Caused by Arthritis}

MCs play a critical role in the pathogenesis of synovitis in a murine model of rheumatoid arthritis (RA) $[38,39]$. The synovium of patients with RA is chronically inflamed and characterized by an expanded population of MCs, as in the mouse model. MCs are markedly increased in number and can constitute $\geq 5 \%$ of the expanded population of total synovial cells. The number of accumulated MCs differs substantially from patient to patient, in general, varying directly with the intensity of joint inflammation [40-42]. MC mediators (histamine and tryptase) are also present at higher concentrations in the synovial fluid of inflamed human joints [43].

MC degranulation has long been associated with arthritis in several animal models, but a critical functional role in the disease was established in the $\mathrm{K} / \mathrm{BxN}$ mouse model $[39,44]$. This arthritis model closely mimics human RA via symmetric joint 
involvement, chronicity, a distal-to-proximal gradient of joint involvement and histological features, including synovial infiltrates, pannus, and erosions of cartilage and bone [45]. Mice deficient in MCs are highly resistant to arthritis, whereas reconstitution with normal MCs restores the wild-type phenotype. Furthermore, degranulation of MCs in the synovium is the first event observed histologically, occurring within $1-2 \mathrm{~h}$ of administration of $\mathrm{K} / \mathrm{BxN}$ serum [46]. Recent studies reaffirmed a role for $\mathrm{MC}$ mediators in arthritis [38]. Thus, MCs are a normal cell population within the human synovium and have a critical role in the pathogenesis of inflammatory arthritis.

The mitochondrial electron transport chain is the machinery that orchestrates one of the most fundamental chemical processes; the generation of cellular energy from oxygen resulting in the fuel that supports all eukaryotic life. However, it is a highly sensitive process and, when unbalanced, leads to the generation of free radicals or ROS, which have been proposed as being the mechanism underlying many chronic human diseases, including MC activation and inflammatory arthritis [47]. Fullerenes can be specifically designed to target and accumulate in the internal mitochondrial membrane bilayers. Once incorporated, FDs are positioned to neutralize superoxide molecules, reactive lipid radicals and radicals that have formed on transmembrane proteins at the site where they are generated, thus, inhibiting inflammation $[15,21,48]$. This, in turn, is predicted to impact diseases whose pathologies stem from radical injury.

Therefore, rationally designed FDs were synthesized to significantly block ROS production $[32,49]$. While it has been shown previously that human MC degranulation in response to FceRI stimulation involves ROS [45], it is not clear whether blocking ROS 
directly blocks degranulation and cytokine production. However, results suggest that blocking ROS using FDs in response to an immune complex (a FceRIIA-dependent stimuli [50]) parallels inhibition of mediator release. This is in line with previous work suggesting that FDs interfere with the generation of mitochondrial-derived ROS [51, 52].

To investigate the ability of FDs to inhibit arthritis, the $\mathrm{K} / \mathrm{BxN}$ serum transfer model was used. This model induces a rapid and severe synovitis, similar to human RA, which is dependent on neutrophils, MCs and macrophages. Its MC dependence was revealed from studies in which two strains of mice deficient in this cell type, W/Wv and Sl/Sld, were resistant to disease induction following serum transfer [44]. Susceptibility to the disease is restored in the $\mathrm{W} / \mathrm{Wv}$ strain by $\mathrm{MC}$ engraftment. Thus, in the $\mathrm{K} / \mathrm{BxN}$ model, MCs function as a link between the serum transfer induction of autoantibodies, soluble mediators and other effector populations. By contrast, MC-deficient mice are still susceptible to collagen-induced arthritis [53] as well as anticollagen antibody/lipopolysaccharide-induced arthritis [45].

Previously published results have demonstrated the ability of FDs to inhibit MCmediated [20, 22] and general inflammation [15]. Given that MCs are the cellular link leading to inflammatory arthritis induction and progression, it has been hypothesized that blocking MC mediator release could block inflammatory arthritis. The liposomeencapsulated FD amphiphilic liposomal malonylfullerene (Figure 2.4) was administered intraperitonealy before disease induction using the $\mathrm{K} / \mathrm{BxN}$ model [44], and during the course of disease onset there was a striking inhibition of the $\mathrm{K} / \mathrm{BxN}$-induced disease pathology. After $\mathrm{K} / \mathrm{BxN}$ serum transfer, the serum-treated mice demonstrated typical 
synovial hyperplasia, pannus formation and inflammatory infiltrates (Figure 2.5A). By contrast, amphiphilic liposomal malonylfullerene-treated animals had less evidence of clinical joint inflammation (Figure 2.5B) comparable to nondiseased animals (Figure 2.5C).

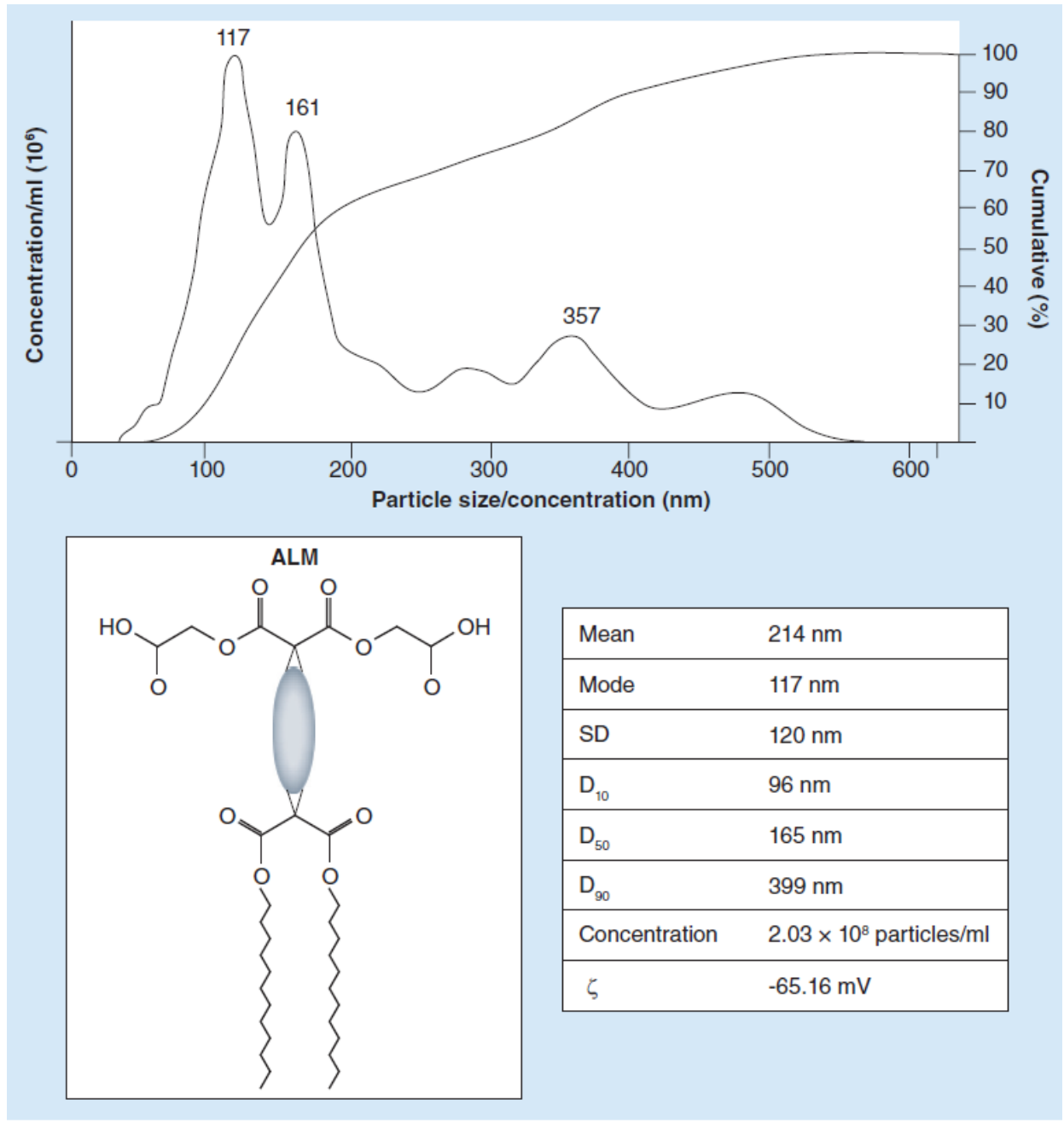

Figure 2.4. Characterization of the Novel Fullerene Derivative Amphiphilic Liposomal Malonylfullerene $\left(\mathrm{C1}_{04} \mathrm{H}_{56} \mathrm{O}_{12}\right)$. ALM: Amphiphilic liposomal malonylfullerene; $\mathrm{D}_{10}$ : Diameter where $10 \%$ of the population have diameters below that size; $D_{50}$ : Median diameter where half of the population have diameters below that size; $D_{90}$ : Diameter where $90 \%$ of the diameters are below that size; SD:

Standard deviation. 

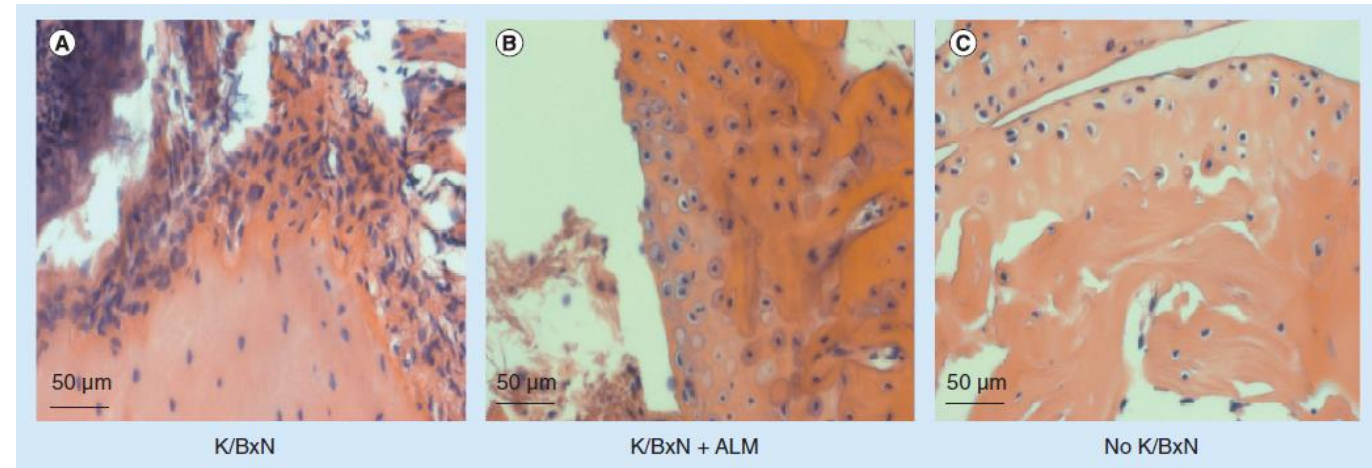

Figure 2.5. Fullerenes Attenuate Inflammatory Arthritis of the $\mathrm{K} / \mathrm{BxN}$-Induced

Disease Pathology. (A) Serum-treated mice demonstrated typical synovial hyperplasia, pannus formation and inflammatory infiltrates. (B) By contrast, ALMtreated animals had less evidence of clinical joint inflammation compared with $(\mathrm{C})$ nondiseased animals. ALM: Amphiphilic liposomal malonylfullerene.

\section{II.6 Fullerenes as a Potential Therapy for MS}

Experimental allergic encephalomyelitis (EAE) is a rodent model of human MS characterized by inflammation in the CNS [54]. Similar to human MS, EAE is associated with an early breach of the blood-brain barrier (BBB), focal perivascular mononuclear cell infiltrates and demyelination leading to paralysis of the extremities. While CD4+ T cells have been implicated, the underlying cause of increased vascular permeability that facilitates the entry of $\mathrm{T}$ cells into the CNS is unknown.

MC contribution to the pathogenesis of MS has been hypothesized based on their presence in CNS plaques of MS patients and the correlation between the number, distribution or MC markers in MS or EAE pathology [55]. Further evidence for MC involvement in EAE/MS came from studies using MC-deficient mice [56]. The MCdeficient $\mathrm{W} / \mathrm{Wv}$ mice exhibited significantly reduced disease incidence, delayed disease onset and decreased mean clinical scores when compared with their wild-type congenic 
littermates. No differences were observed in the T- and B-cell responses between the two groups, and reconstitution of the $\mathrm{MC}$ population in $\mathrm{W} / \mathrm{Wv}$ mice restores induction of early and severe disease to wild-type levels. These data provide a new mechanism for immune destruction in EAE and indicate that MCs may be sentinels of neurologic inflammation.

Previous studies have demonstrated that fullerene-based therapeutics can significantly ameliorate EAE [7]. The FD protected neurons from oxidative and glutamate-induced injury, and restored glutamine synthesis and glutamate transporter expression in astrocytes under inflammatory insult. The in vitro efficacy translated into in vivo efficacy, as treatment initiated after disease onset reduced the clinical progression of chronic EAE in mice, suggesting this may be useful in the treatment of progressive MS and other neurodegenerative diseases.

A fullerene-based MS therapeutic was designed to specifically target axonal cells. Here a MC-stabilizing FD was conjugated with tropine and an IR800 dye for tracking [22]. This MC-stabilizing compound is targeted to neurons based on the affinity of tropine (a derivative of tropane containing an oxygen atom) for neuronal cell dopamine transporter receptors [57]. Thus, in addition to its ability to inhibit MC-mediator release, this compound is theorized to also target neurons and scavenge ROS. To determine if this MC-stabilizing, neuron-targeting, IR800 dye fullerene molecule could cross the BBB, mice were injected intravenously with fullerene dye, and imaged immediately and at several time points. 
Initial experiments show the fullerene-dye conjugate can cross the BBB and be deposited in the brain (Figure 2.6). This approach using basic chemistry to add a variety of axon/neuron-targeting motifs with varying ratios of targeting motif to the fullerene core is now being used to foster maximal cell targeting and uptake. These results confirm previous studies using a $\mathrm{C}_{60}$-based formulation suggesting FDs can cross the BBB. First, mice orally treated with FDs have reduced superoxide generation in the brain [58].

Second, FDs given orally can reverse age-related changes in mitochondrial electron transport function and block age-related increases in superoxide produced by mitochondria taken from the brain $[17,59]$.

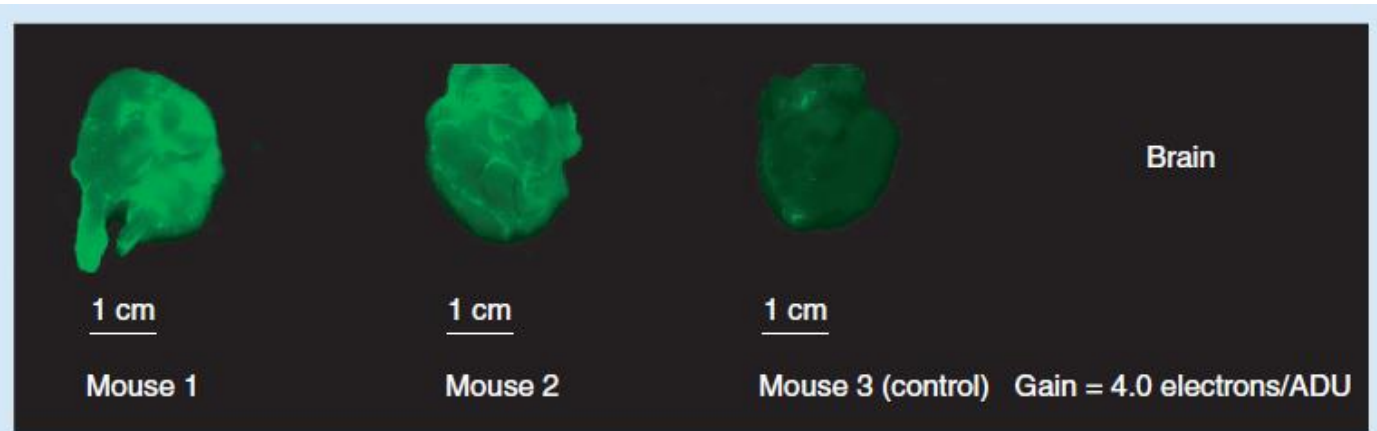

Figure 2.6. A Neuron-Targeting Fullerene Crosses the Blood-Brain Barrier. Mice 1 and 2 were injected with $0.5 \mathrm{ml}$ of the IR800 dye-labeled fullerene by tail vein 15 min apart. Mouse 3 is a control (no dye). The brain was removed at 6 h, sectioned and scanned.

The SH-SY5Y human neuroblastoma cells express dopamine transporters and were differentiated into nondividing neurons using a protocol described previously [60]. A Texas Red ${ }^{(}$(Life Technologies, CA, USA)-conjugated, fullerene-tropane derivative clearly recognized the neuronal dopamine transporter receptors (Figure 2.7A). This is 
evident by the accumulation of clusters of red (from the Texas Red dye) on the membrane of the neurons and also staining of the neuronal axon. Interestingly, it appears there is an abundance of fullerene-tropane at the newly formed part of the axon (closest to the cell core) but not towards the outer axon sheath. Fullerene-tropane also penetrates into cells where there is evidence of mitochondrial colocalization (Figure 2.7B).
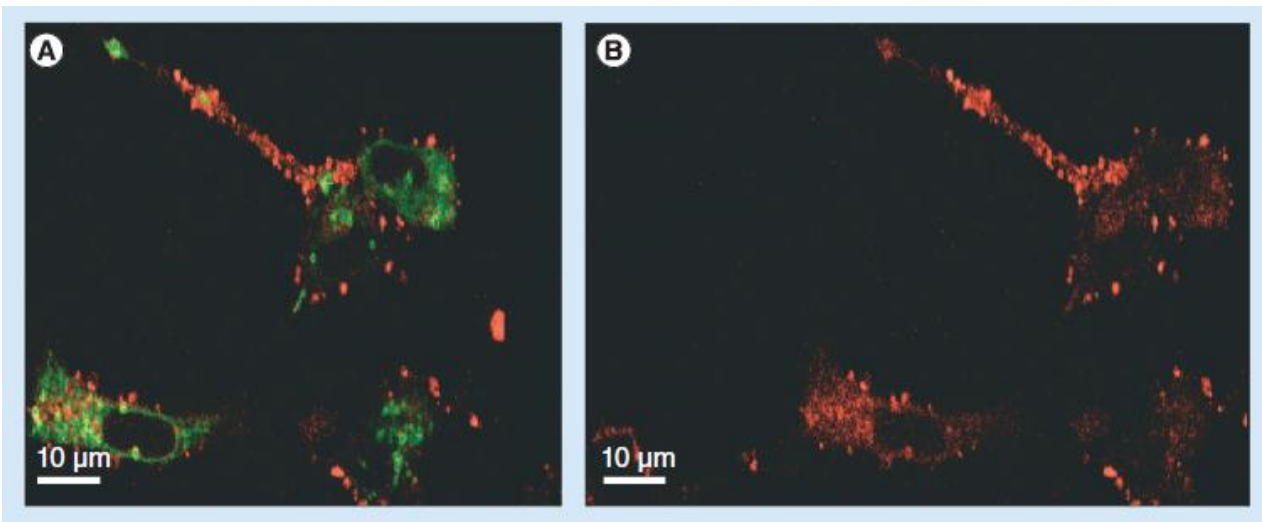

Figure 2.7. Fullerene-Tropane Derivatives can Target the Neuronal Dopamine Transporter Receptors on Human Neuroblastoma Cells. A neuron-targeting fullerene derivative (A) colocalizes with mitochondria and (B) selectively binds neuronal cells through tropane receptors.

MCs are important regulators of MS [54-56]. Oxidative stress, through the generation of ROS, is an underlying mechanism that mediates MC signaling and MS pathology [61]. Indeed, several antioxidants are currently in various phases of human clinical trials (i.e., lipoic acid, inosine and Triomar ${ }^{\circledR}$ [Pronova Biocare, Oslo, Norway], see [62]). Given that FDs can stabilize MCs [22], are potent antioxidants $[49,63]$ and are anti-inflammatory medications $[15,48]$, FDs were tested for their ability to inhibit EAE. As seen in Figure 2.8, FDs reduce the clinical scores associated with MS in the EAE 
model, suggesting this class of rationally designed compounds may be used as a platform for new areas of therapeutic research for MS.

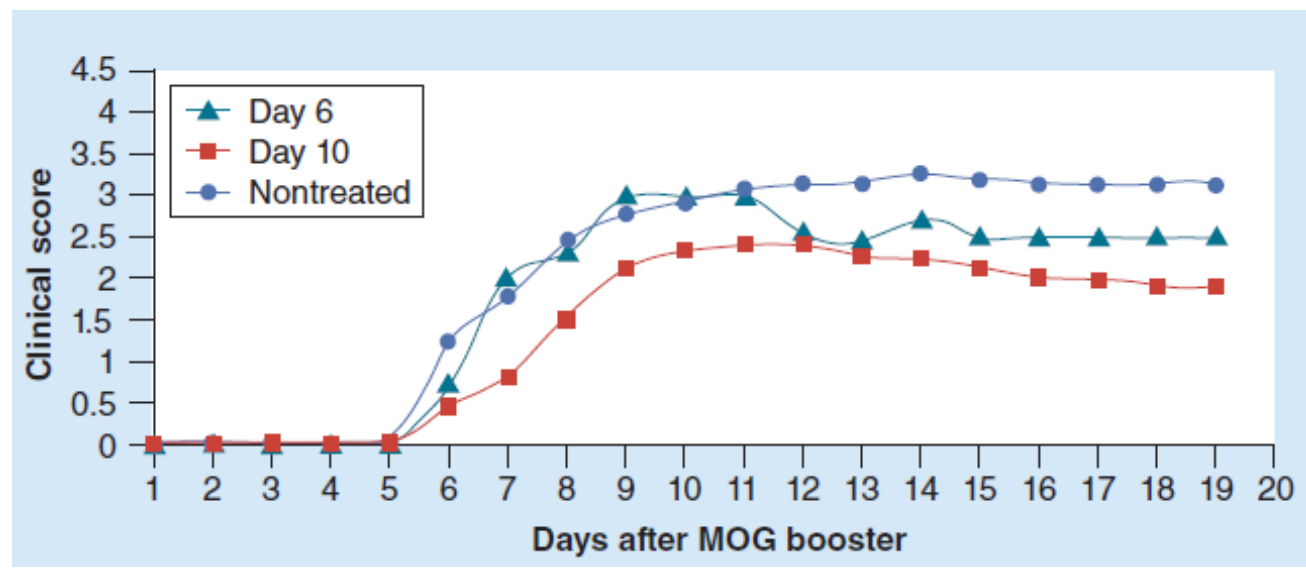

Figure 2.8. Fullerene Derivatives Inhibit the Onset of Experimental Allergic Encephalomyelitis. Fullerene derivatives inhibit the onset of experimental allergic encephalomyelitis in mice assessed at day 6 (triangles) and day 10 (squares) compared with nontreated animals (circles).

\section{II.7 Fullerenes as Diagnostics}

Carriers for diagnostics have shown great potential in solving many present day medical imaging limitations. Endohedral fullerenes consist of a hollow core carbon shell, which during synthesis is capable of encapsulating metal ions within the cage. The endohedral fullerene cage is typically larger than the common $\mathrm{C}_{60}$. This is due to size constraints imposed by the small size of the $\mathrm{C}_{60}$ molecule; therefore, $\mathrm{C}_{80}$ molecules (or even higher: $\mathrm{C}_{82}, \mathrm{C}_{84}, \mathrm{C}_{86}, \mathrm{C}_{88}$ and $\mathrm{C}_{2 \mathrm{n}}$; i.e., $\mathrm{n}=12,13,14$ and so on) accommodate sufficient enclosure of desired metals, including lanthanum, yttrium, scandium and gadolinium. The endohedral fullerene's strong electronic properties can strongly enhance diagnostics. Such compounds are currently being investigated as a platform to detect 
cancer [64], atherosclerosis [65] and arthritis [66]. Present day diagnostic limitations of both sensitivity and specificity have stalled progress on accurate, enhanced patient imaging. Thus, metal endohedral fullerenes have the potential to affect the diagnosis of numerous other diagnostic pathologies.

One class of endohedral fullerene is typified by Trimetaspheres ${ }^{\circledR}$ (TMS; Luna Innovations Incorporated, VA, USA), which are $\mathrm{C}_{80}$ based with gadolinium enclosed within the cage (Figure 1.1C). One field in which they are being investigated for use is in MRI - a widely used diagnostic procedure that utilizes gadolinium-based contrast agents (i.e., Magnevist ${ }^{\mathrm{TM}}$; Bayer HealthCare Pharmaceuticals Inc., NJ, USA). However, several issues have limited gadolinium chelate-based contrast agents (i.e., Magnevist) for use as image-guided interventions with MRI, including gadolinium toxicity [67-70], rapid clearance from the body, poor relaxivity-dependent sensitivity and inability to be targeted to disease-specific biomarkers. The TMSs solve many of the problems associated with current gadolinium-based contrast agents. First, the gadolinium (inside cage) that is toxic at sufficient concentrations is separated from active targeting moieties (outside cage) by an extremely stable carbon shell, possibly increasing safety. Unlike current chelates, adding targeting ligands/moieties to TMSs does not affect the ability of gadolinium to become free of the compound. Second, gadolinium-encapsulated endohedral fullerenes are more sensitive. Targeted imaging agents require strong signals by which to report the presence of an agent at a particular location. The TMS achieves 25- to 50-fold greater relaxivity compared with traditional gadolinium contrast agents. Third, the fullerene cage can be targeted to disease biomarkers, as targeting moieties can be attached to the cage 
(including empty cage fullerenes). Finally, the TMSs appear to be safe; no toxicity was detected in vitro and in vivo $[49,71]$, although further studies to assess their safety are clearly needed.

The crux of the TMS platform for diagnosis utilizes specific biomarkers conjugated to the gadolinium-encapsulated endohedral fullerene MRI contrast agents and, in theory, could be used for any disease for which specific expression of suitable receptors exist. Two disease processes evaluated, atherosclerosis and glioblastoma, possess disease-specific biomarkers that can be targeted. Based on working prototypes, TMS endohedral fullerenes have been developed to study their cellular uptake by macrophage foam cells (for atherosclerosis) and glioblastoma cells (for cancer) in vitro. This prototype takes advantage of already identified biomarkers on diseased cells, followed by chemically attaching ligands to target the TMS to these cells (characterizing the molecules), and use of in vitro assays to determine if they bind to and enter the cells and assess any toxicological effects.

\section{II.8 Endo-Fullerene Contrast Agents as Diagnostics for Atherosclerotic Plaque}

Atherosclerosis is an inflammatory disease representing a major healthcare problem in the USA. Initiation of atherosclerosis begins when blood cells (monocytes) 'stick' to blood vessel walls as a result of an individual's increased cholesterol consumption. Over time the cells continue to accumulate on vessel walls and engulf the fat droplets until large structures called plaques begin to form. The plaque can rupture and lead to complete blockage of blood flow through the vessels involved, leading to a 
heart attack and/or stroke. To be able to noninvasively determine the extent of plaque build up in vessel walls of at risk patients using already established diagnostic procedures would facilitate treatment options that could greatly reduce myocardial infarction and stroke incidence, which represent the most frequent causes of death in western society. While several molecules have already been identified that are selectively expressed or highly upregulated on plaque lesions [72-74], preliminary findings in the field focus on CD36 (macrophage scavenger receptor) as a potential target receptor. The CD36 receptor is involved in foam cell formation, mediating the influx of lipids into macrophages, possessing a promiscuous ligand binding and is highly expressed on lipid-laden macrophages of human atherosclerotic aorta [75-78]. Different forms of oxidized phosphatidylcholine and oxidized low-density lipoprotein are high affinity ligands for CD36 [79]. The basic structure of the compounds consists of the TMS enclosed within liposomes, which was previously demonstrated [48], with CD36 ligands decorated on the liposomes (Figure 2.9). As seen in Figure 2.10, APOE knockout mice [80] were used to test the atherosclerotic plaque-targeting contrast agent, which serves as a working prototype for a new class of diagnostic agents that specifically attach to atherosclerotic plaques, so that previously undetected lesions can be diagnosed using MRI. The atherosclerotic plaque-targeting contrast agent clearly reveals plaque accumulation by MRI that is not seen using the same molecule without targeting ligands. 


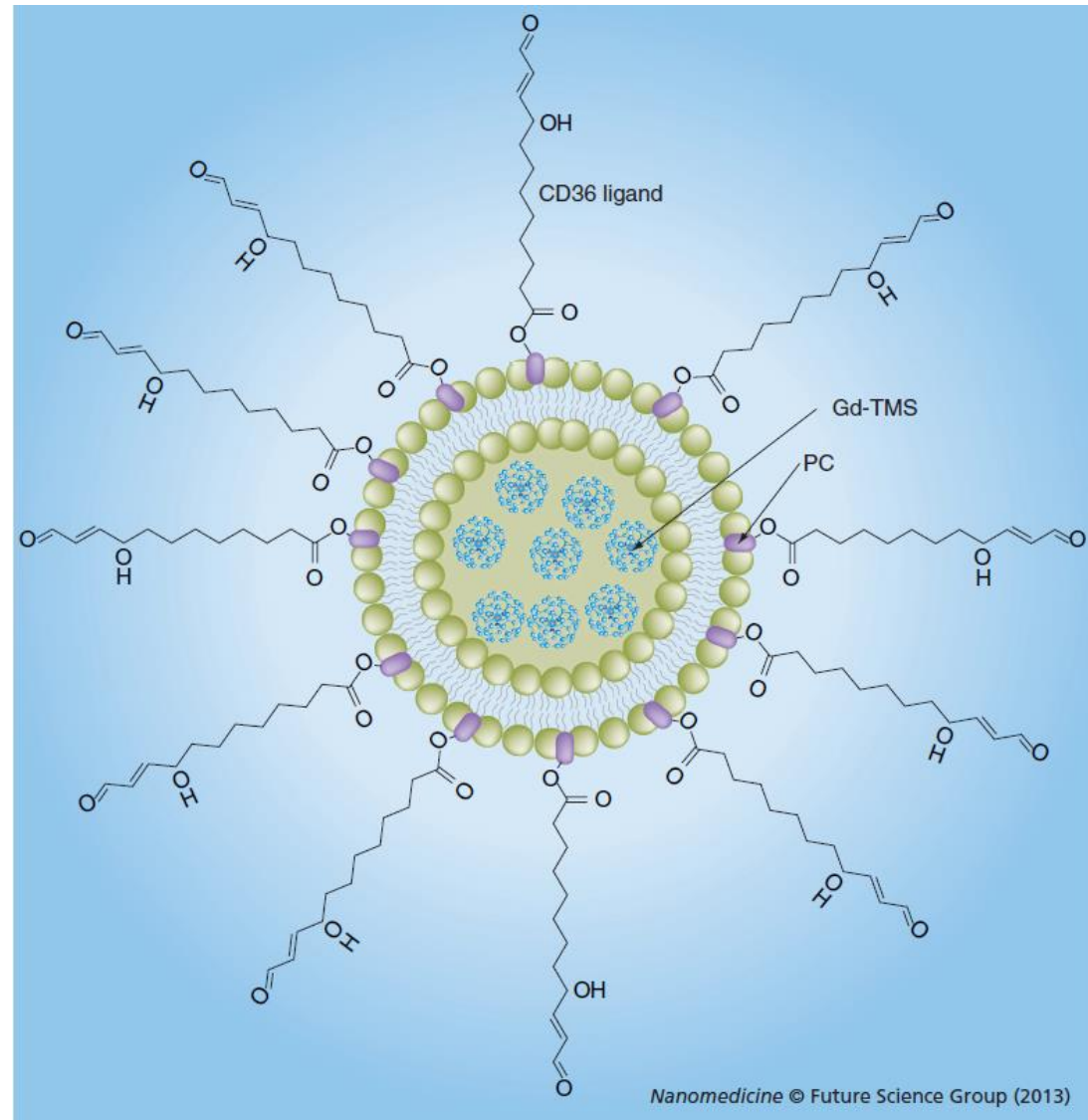

Figure 2.9. Basic Structure of Atherosclerosis-Targeting Contrast Agents. Not drawn to scale. Gd-TMS: Gadolinium Trimetaspheres®; PC: Phosphatidylcholine. 


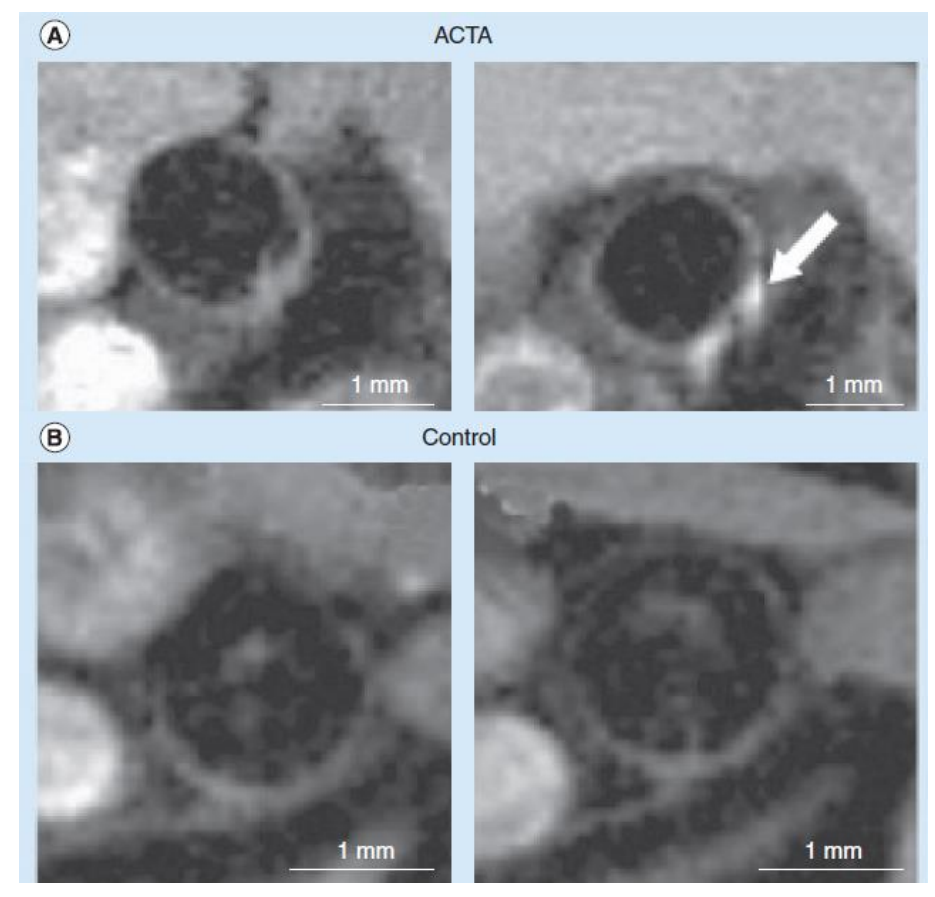

Figure 2.10. Atherosclerotic Plaque-Targeting Contrast Agents can Detect Plaque In Vivo. ApoE-/- with plaque were imaged using MRI. The (A) ATCA or (B) nontargeted controls were injected intravenously and images of the descending aorta acquired before or $120 \mathrm{~min}$ after. The arrow indicates the ATCA binding to the plaque in the blood vessel, while the controls do not. ATCA: Atherosclerotic plaque-targeting contrast agent.

\section{II.9 Fullerenes as Theranostics}

Multifunctional approaches that incorporate both medical imaging and therapy could be the future of medicine, and fullerenes prove to be an attractive platform for these approaches. This is due to the fullerene's ability to simultaneously detect (with biomarkers), image (with gadolinium-based contrast) and deliver a therapeutic payload to a specified site. Progression can be monitored in real time to assess the effectiveness of treatment using direct visualization of the areas of interest through the high-contrast capabilities of endohedral fullerenes. Nanoparticles are presently being pursued for imag- 
ing and diagnostic applications $[81,82]$ and the next logical leap would be to couple this with patient therapy. Such multifunctional nanoplatform-based probes have shown to be effective in mouse models, including tumor detection and treatment [83-85], as well as atherosclerosis and arthritis [86-88].

\section{II.10 Endo-Fullerene Contrast Agents as Theranostics for Brain Cancer}

Glioblastoma multiforme is an aggressive high-grade brain tumor with a poor prognosis. Ideally, a multifunctional nanoscale compound is needed that can simultaneously diagnose tumor progression and can also specifically target and kill the tumor. IL-13 receptors are selectively expressed on astrocytoma cells in gliomas. It has been previously reported that IL-13 receptor-targeted chemotherapies (diphtheria toxin) $[89,90]$ delivered through lipid nanovesicles (liposomes) were effective in targeting and shrinking glioma tumors in a subcutaneous tumor model. MRI using gadolinium-based contrast agents has been used for diagnosing glioblastoma. IL-13 receptors are expressed on glioblastoma multiforme cells and are not significantly expressed in normal tissue [91]. In fact, many clinical trials are underway using IL-13 as a targeting moiety for various interventions of the disease [62]. TMS was used as a platform for developing novel glioblastoma-targeting theranostics in which the diagnostic TMS is enclosed within glioblastoma-targeting IL-13 liposomes that can deliver a therapeutic payload of doxorubicin (Figure 2.11).

After verifying in vitro binding of the glioblastoma-targeting theranostics to human glioblastoma cells, the nanoparticles were tested in vivo. As seen in Figure 2.12, 
the glioblastoma theranostic can target and shrink human brain tumors that have been transplanted in mice.

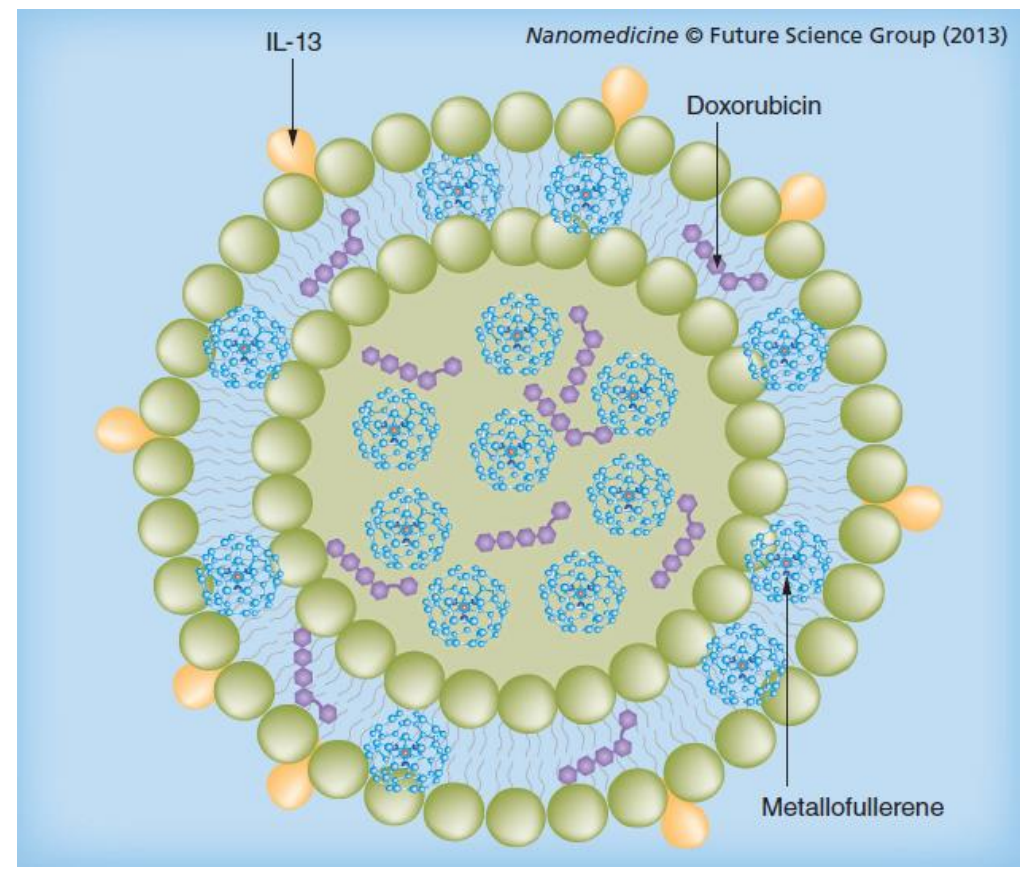

Figure 2.11. Glioblastoma-Targeting Theranostic. The Trimetaspheres ${ }^{\circledR}$ (Luna nanoWorks, VA, USA) are incorporated within and/or in the liposome bilayer along with doxorubicin and are targeted to the glioma cells by IL-13 (not drawn to scale). 


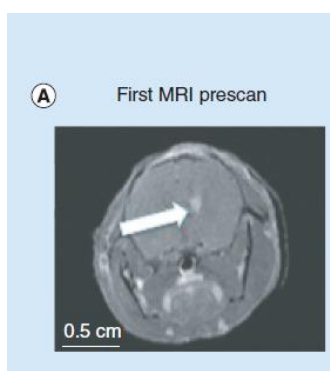

(B)

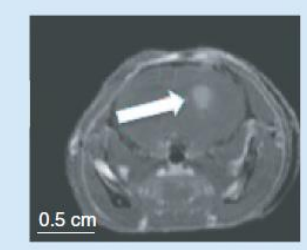

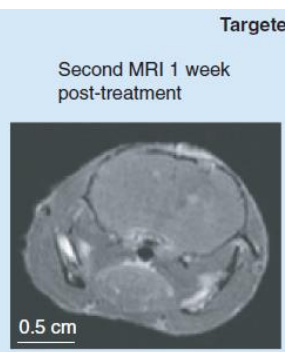

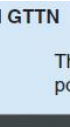

Third MRI 2 weeks
post-treatment

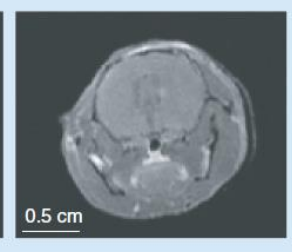

Nontargeted nanovesicles (control)

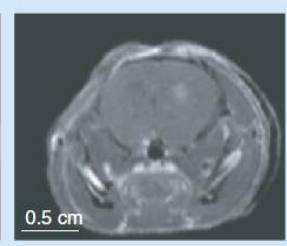

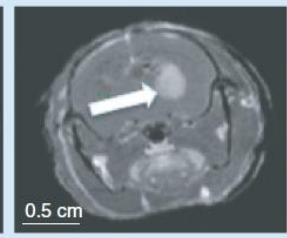

Fourth MRI 3 weeks

post-treatment
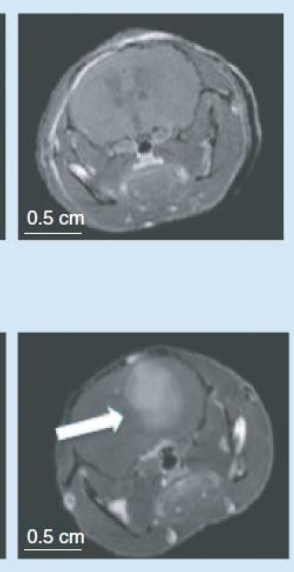

Figure 2.12. Glioblastoma-Targeting Nanovesicles with a Therapeutic Payload Attached can Shrink Brain Tumors. Mice with human glioblastoma cells injected into the brain have uncontrolled growth (white mass). (A) When mice are injected with targeted nanovesicles the tumors shrink and disappear over time (as determined by MRI). (B) However, tumor size increases significantly over time in mice injected with nontargeted nanovesicles. GTTN: Glioblastoma-targeting theranostics.

\section{II.11 Fullerene Toxicity}

Of course, toxicity considerations are implicit when contemplating human use for novel compounds such as fullerenes. To this end there have been a number of studies examining the toxicity using a myriad of fullerene preparations. However, the results of most of these studies are conflicting, inconclusive and the subject of much debate. One such fullerene study (which was subsequently proven to be unfounded) exposed juvenile bass to nonderivatized $\mathrm{C}_{60}$, which is insoluble in water [92]. Unfortunately the authors did not include a control that provided insights into whether the observed effects were simply due to large aggregated particles, tetrahydrofuran contaminants or whether they were specific to the chemical nature of $\mathrm{C}_{60}$. However, there was widespread publicity that 
concluded fullerenes as a class of compounds could be toxic [93]. A subsequent publication by the same group (without publicity) demonstrated that the originally observed 'toxicity' was due to impurities in the sample [94]. Even more recently these original studies were formally addressed by a follow-up publication, which stated that the original Oberdorster study was 'compromised by experimental artifacts' [95]. On the opposite end of the spectrum, studies in mice demonstrate that similar $\mathrm{C}_{60}$ preparations significantly increase the lifespan of mice [17] and rats [18]. Thus, it is difficult for researchers, and the general public, to determine if fullerenes are dangerous nanostructures that should be banned or a potential novel platform for developing new medicines. The answer to explain how a class of compounds can be simultaneously toxic and lifespan extenders will rely on well-controlled structure-activity studies.

Contributing to the confusion is that few studies examining toxicity use well characterized and highly purified material; those that are not mixtures of many isomers, aggregate sizes and impurities. Most studies in which fullerenes are deemed to be toxic use starting material with little or no characterization (e.g., dynamic light scattering, zpotential and Fourier transform infrared spectroscopy). In addition, no studies have compared differences in cage sizes $\left(\mathrm{C}_{60}\right.$ vs $\mathrm{C}_{70}$ vs $\left.\mathrm{C}_{80}\right)$; the latter two are much more likely to become US FDA-approved products owing to their size being conducive to fewer numbers of isomers when adding side-chain moieties. The FDA requires that every new chemical entity must be evaluated separately; extrapolating toxicity (or nontoxicity) by categorizing compound mixtures and making generalizations about classes of compounds (as is the case with fullerenes) with many different isomers is not acceptable 
to the FDA. A fundamental understanding of basic chemistry reveals that even extremely similar molecules can have different biologic activities. There are many examples where two isomers that are very similar have completely different biological behavior. For example, the tragedy of the drug thalidomide, where one isoform was an effective sedative and the mirror image isoform was teratogenic (resulting in fetal defects), changed the face of drug testing. It is important to note that pristine nonfunctionalized fullerenes behave significantly differently to their water-soluble functionalized counterparts, furthermore each variation in fullerene functionalization should be treated and evaluated as a separate entity. This applies to the studies with fullerenes, where even extremely small changes in the core fullerene structure (through the addition of sidechain moieties) can result in the FD having completely different biological properties. Several laboratories have demonstrated this repeatedly, which highlights the difficulty in interpreting data gathered from extrapolating findings between even very similar compounds.

Complicating the task of bringing FDs to the marketplace is the fact that the FDA does not have specific guidelines for products containing nanoscale materials. A report issued by the FDA Nanotechnology Task Force (July 2007) recommends guidance by various centers within the FDA for industries working with nanomaterials [96]. Unlike 'standard' drug products, it is increasingly evident that at least in the area of characterization of nanomaterials used in drug products different standards apply. Applying small molecule principles and methodologies to nanomaterials cannot be extrapolated in biological settings. The study stressed that biodistribution analysis should 
be at the core of any evaluation of products containing nanomaterials. These biodistribution studies, as recommended by the FDA [96], provide valuable information on where the nanoparticles are traveling and possibly accumulating, therefore, subjecting those sites to increased likelihood of toxicological effects. It was also stressed in the 2007 report that most studies (using nanomaterials) are limited in that they are short-term and might leave long-term effects unevaluated, especially because the long-term toxicity and effects for most nanoscale materials remain unknown. Furthermore, appropriate end points for in vitro assays are seen to be difficult to determine, as single cell types are often not sufficient for evaluation on the function or health of organs or tissues that are made up of multiple cell types, given that FDs are exposed to numerous types of tissues in the body. The major recommendation from the reports was that nanoscale materials be characterized with respect to size (surface area and size distribution), chemical composition (i.e., purity and crystallinity), surface structure (e.g., surface reactivity, surface groups and coatings), solubility, shape and aggregation. The protocols developed at the National Cancer Institute's, Nanotechnology Characterization Laboratories (MD, USA) was recommended as being very useful in helping to characterize nanoscale materials, and to develop standards and standardized methods for measuring nanoscale materials [97].

At present, it is difficult to make conclusions on the absorption, distribution, metabolism and excretion profile of the many types of carbon nanomaterials presently being investigated: carbon nanotubes; nanohorns; graphene; fullerenes; and derivatives of each of these nanomaterials. This is primarily due to the relative infancy of the field and 
limited research on the long-term effects of fullerenes on biological systems. However, several studies have been conducted assessing the elimination of fullerenes from the body $[19,98]$. Researchers have concluded that there is limited absorption of fullerenes following both intravenous and oral exposure in rats. In general, elimination was rapid from the body via feces and urine $[19,98]$. Furthermore, several experimental studies revealed that mice treated with excessive dosages $(2000 \mathrm{mg} / \mathrm{kg})$ of fullerenes have shown no overt toxicity $[99,100]$. In fact, some studies have shown that fullerene treatment in mice and rats can improve cognitive function and extend lifespan by $11 \%$ (in the mouse model) and $90 \%$ (in the rat model) $[17,18]$. Clearly contrasting results and limited long-term data need to be addressed and efforts on creating reliable, reproducible results are still required for successful translation of these molecules into human systems. Focusing research to clarify chronic exposure to these materials, as well as identification of genotoxicity, carcinogenicity and reproductive toxicity will be paramount for encouraging fullerene studies in biological systems. Certainly, each carbon nanomaterial and specific derivatives should be carefully evaluated and characterized individually, as these materials exist in a variety of forms (e.g., carbon structure, carbon number, surface modification, charge, size, aggregation and degree of functionality). The extrapolation of results across such broad classes is not sufficient in characterizing the toxicity profiles of these molecules.

Future research should be aimed at devising appropriate testing strategies to evaluate risk and toxicity profiles that do not generalize carbon nanoparticles as one, but rather evaluate each class individually. 


\section{CHAPTER III}

\section{FULLERENES INHIBIT ALLERGIC MEDIATOR RELEASE FROM HUMAN MAST CELLS AND BASOPHILS}

\section{III.1 Introduction}

Mast cells (MC) are ubiquitously expressed in almost all tissue and participate as effector cells for immune regulation. Peripheral blood basophils (PBB) are similar to $\mathrm{MC}$ in that they have pre-stored, allergy inducing mediators in their granules (e.g. histamine). Once stimulated, MC/PBB secretes several molecules, including preformed and newly formed inflammatory mediators, via various physiological and nonphysiological stimulations $[30,101,102]$. While the classical IgE/FceRI pathway is the most well studied and understood pathway leading to $\mathrm{MC} / \mathrm{PBB}$ mediator release, these cells can be stimulated by non-IgE secretagogues.

Indeed, non-IgE stimulation may be more physiologically relevant in non-allergic conditions such as innate immunity and heart disease [30, 63, 103]. Thus, finding new ways to stabilize these cells as a strategy for controlling MC/PBB-diseases is a continuous need. Fullerenes, which are nanometer-sized tiny spherical carbon cages, are being explored in a wide array of applications including nanomedicine $[63,104]$. The carbon cage is insoluble without the addition of appropriate side chains that confer water solubility, an important requirement for medical applications. Recent studies suggest that water-soluble fullerene derivatives (FD) can inhibit FceRI-induced MC responses in vitro, which translates to the prevention of MC-driven anaphylaxis and asthma in vivo 
$[20,22,32]$. Although it is clear certain FD can stabilize MC activation through FceRI in vitro and in vivo it is not known if they can stabilize MC activation through non-FceRImediated stimuli. In these studies, the non-Fc\&RI/IgE mediated inhibitory effects of a panel of FD were tested for their ability to stabilize MC/PBB. It is shown that incubation of $\mathrm{MC} / \mathrm{PBB}$ with fullerene constructs can significantly inhibit mediator release in response to various secretagogues. This inhibition was mediated in part through reductions in the generation of reactive oxygen species (ROS), cellular fluctuations of calcium, and phosphorylation of signaling molecules in activated MC. It is demonstrated that FD inhibit non-IgE mediated pathways and the efficacy of FD relies on several factors: the active side chain moieties added to the fullerene cage, the type of cells evaluated, the secretagogue used to stimulate, and the pathway that is examined.

\section{III.2 Methods}

\section{III.2.1 Fullerene derivatives}

Luna Innovations Incorporated synthesized all FD. Each FD was characterized using matrix assisted laser desorption ionization mass spectrometry (MALDI-TOF), nuclear magnetic resonance (NMR), and high performance liquid chromatography (HPLC). All FD used were between 1 and $50 \mathrm{~nm}$ in aqueous solution as determined by dynamic light scattering (DLS) using a Malvern Zetasizer Nano-S90 and a NanoSight LM10 with NTA Software allowing individual particles to be tracked and characterized. To evaluate fullerene-specific toxicity, $\mathrm{MC}$ viability was determined by incubation with concentrations up to $100 \mu \mathrm{g} / \mathrm{ml}$ over nine days (a 10x higher concentration and longer 
time period than was needed to control cellular responses to stimuli). No significant $(p<0.05)$ toxicity was observed with any of the selected compounds when compared to controls as determined using trypan blue staining and MTT Assay.

\section{III.2.2 Human mast cell and peripheral blood basophil cultures and activation}

Human tissue (i.e. skin) was received from the Cooperative Human Tissue Network. All MC studies were approved by their Human Studies Institutional Review Board. Mast cells were purified and cultured as described $[105,106]$. Peripheral blood basophils were obtained from normal donors (no medications) after informed consent as approved by the Institutional Review Board, Johns Hopkins University and purified to $>90 \%$ as described previously [107].

A panel of FD was screened at several concentrations to determine their ability to inhibit secretion. Briefly, $\mathrm{MC} / \mathrm{PBB}$ were first incubated with increasing concentrations of FD $(1-40 \mu \mathrm{g} / \mathrm{ml})$ overnight $(18 \mathrm{~h})$ at $37^{\circ} \mathrm{C}$ and $6 \% \mathrm{CO}_{2}$, a time point and concentration range found to be optimal for cellular uptake of FD [21, 22]. The next day, cells were stimulated with the indicated secretagogues at varied concentrations for $30 \mathrm{~min}$ (degranulation and PGD2) or overnight (cytokine production) at $37^{\circ} \mathrm{C}$ and mediator release measured as described previously or according to the manufactures instructions (Cayman Chemical Company, Ann Arbor, MI, Prostaglandin D2 Kit-512031). The percent inhibition was calculated as a percent release compared to non-FD treated cells (positive control). An initial dose response was performed to determine the concentration of secretagogues that induced maximal degranulation/cytokine production (not shown). 
In short, $\mathrm{MC}$ responded optimally to $10 \mu \mathrm{M}$ with $\mathrm{A} 23187$ and compound 48/80, $20 \mu \mathrm{M}$ for somatostatin, and $40 \mu \mathrm{M}$ for poly L-lysine. Other secretagogues (C5a, morphine, substance P, and LPS) were examined, however preincubation with FD did not produce statistically significant reductions in MC mediator release or stimuli did not induce significant activation (data not shown). For PBB, $10 \mu \mathrm{M}$ was optimal activation with fMLP, poly L-lysine, and A23187 (data not shown). All studies were performed in triplicate on at least three separate MC cultures or PBB donors.

\section{III.2.3 Reactive oxygen species and calcium measurements}

Human skin MC $\left(2 \times 10^{6} / \mathrm{ml}\right)$ were incubated overnight with FD (those found to significantly inhibit degranulation and/or cytokine release), washed, and loaded with DCF-DA (final concentration of $5 \mu \mathrm{M}$ ) for $30 \mathrm{~min}$. Following fluorophore loading, cells were washed and resuspended in fresh media, placed in a cuvette, and activated with various secretagogues (as above) for $\sim 60 \mathrm{~s}$. ROS fluorescence intensity was measured at $523 \mathrm{~nm}$ wavelengths over a 12-minute time interval using Perkin Elmer LS55 Luminescent Spectrometer (Perkin-Elmer Laboratories). For calcium flux, MC (incubated as above) was loaded with Fura-2 AM at a final concentration of $20 \mathrm{ng} / \mathrm{ml}$ for $30 \mathrm{~min}$ at $37^{\circ} \mathrm{C}$ in HBSS buffer. The cells were washed twice in the same buffer and incubated for $15 \mathrm{~min}$. The cells were then stimulated with appropriate secretagogues and a real time ratiometric intensity evaluation of fluorescence between wavelengths 340 $\mathrm{nm} / 380 \mathrm{~nm}$ was determined over a period of $400 \mathrm{~s}$ using Perkin Elmer LS55 Luminescent Spectrometer. All samples were measured in duplicate and performed at least three times. 


\section{III.2.4 Immunoblotting analysis of signal transduction intermediates}

Mast cells ( $1 \times 10^{7}$ cell/condition; each condition performed in triplicate) were prepared for Western blotting as described previously [22]. Briefly, cells were incubated with or without optimal concentrations of the indicated FD overnight, washed, and activated as described above. Cell pellets were lysed and nuclear extracts isolated as described [108]. The cell suspension was heated, passed through a 20 -gauge needle, and centrifuged to remove cell debris. Proteins were separated on $12 \%$ NuPage tris-glycine gels using tris-glycine SDS running buffer. Signaling molecules were measured using phosphorylated MAPK (ERK1 and ERK2) and LAT primary antibodies (Santa Cruz Biotechnology, Santa Cruz, CA or Cell Signaling, Danvers, MA, respectively) and Licor IR-800 anti-mouse $\mathrm{F}(\mathrm{ab}) 2$ secondary antibodies $(1: 20,000)$. The housekeeping protein $\beta$ actin was used as a loading control on the same blot and co-stained with Licor IR-600 anti-rabbit F (ab)2 secondary antibodies. Band intensities were quantified using an Odyssey Imaging System as previous described [22].

\section{III.3 Results}

\section{III.3.1 Fullerenes differentially effect mast cell non-IgE-induced mediator release}

A panel of water soluble FD was evaluated to determine efficacy based on various side chain moieties as well as differential responses to numerous secretagogues. Table 3.1 presents results from a subset $(\sim 30 \%)$ of FD that was capable of significantly $(p<0.05)$ inhibiting MC-degranulation when challenged with 48/80, somatostatin, A23187, and poly-L-lysine. Other secretagogues evaluated included C5a, morphine, substance $\mathrm{P}$, and 
LPS which did not produce significant mediator release from MC or the pathway was unaffected by FD pretreatment, data not shown. These data demonstrate that inhibition of non-IgE-driven MC-degranulation can be generalized across various stimuli, as in the case of $\mathrm{C}_{70}-\mathrm{OH}$ and Tetrasulfonate, or selective to a specific stimuli and/or signaling pathway, as observed in the case of Biotin and CCC.

Table 3.1. Mean \% Inhibition of Degranulation $( \pm$ SD) in FD Treated and Untreated MC.

\begin{tabular}{|c|c|c|c|c|}
\hline $\begin{array}{l}\text { Fullerene } \\
\text { Derivative }\end{array}$ & A23187 + FD & $48 / 80+\mathrm{FD}^{\star}$ & $\begin{array}{l}\text { somatostatin } \\
+\mathrm{FD}^{\star}\end{array}$ & $\begin{array}{l}\text { poly-L-lysine } \\
+\mathrm{FD}^{*}\end{array}$ \\
\hline Biotin & no Inh & no Inh & $44.6( \pm 1.8)^{ \pm=4}$ & no Inh \\
\hline $\mathrm{C} 70-\mathrm{OH}$ & $19.0( \pm 1.1)$ & $42.0( \pm 4.6)^{ \pm x}$ & $13.4( \pm 5.2)^{ \pm}$ & $33.4( \pm 5.5)^{\text {ent }}$ \\
\hline $\mathrm{CCC}$ & no Inh & no Inh & $23.8( \pm 0.8)=$ & no Inh \\
\hline DMAE & $18.6( \pm 7.9)^{ \pm}$ & $16.9( \pm 10 .)^{m}$ & $24.3( \pm 9.7)^{ \pm}$ & no Inh \\
\hline Ethanolamine & $36.0( \pm 3.0)^{2}$ & no Inh & no Inh & no Inh \\
\hline Niacin & no Inh & $59.5( \pm 1.3)^{ \pm x}$ & $21.9( \pm 13.8)^{ \pm}$ & $20.9( \pm 12.6)^{ \pm}$ \\
\hline NSAID & $21.1( \pm 11.4)^{*}$ & no Inh & no Inh & no Inh \\
\hline PC4 & no Inh & no Inh & $21.6( \pm 2.9)=$ & no Inh \\
\hline Tetraglutamate & $22.3( \pm 3.9)^{m}$ & no Inh & $20.3( \pm 4.5)^{ \pm \#}$ & $17.9( \pm 13.1)^{ \pm}$ \\
\hline Tetrainositol & no Inh & no Inh & $32.8( \pm 1.9)= \pm$ & no Inh \\
\hline Tetraphosphate & $18.9( \pm 0.4)^{=}$ & no Inh & no Inh & no Inh \\
\hline Tetrapyridine & $14.0( \pm 1.9)^{ \pm=}$ & $34.8( \pm 8.1)^{ \pm 1}$ & no Inh & $13.1( \pm 4.0)^{ \pm}$ \\
\hline Tetrasłulfonate & $21.1( \pm 6.3)^{ \pm}$ & $44.8( \pm 11.2)^{=1}$ & $22.0( \pm 5.50)^{= \pm}$ & $26.7( \pm 2.3)^{= \pm}$ \\
\hline TGA & $49.8( \pm 8.9)^{\Perp}$ & no Inh & $16.7( \pm 3.8)=$ & $43.8( \pm 10.6)^{=}$ \\
\hline TTA & no Inh & no Inh & $34.6( \pm 2.6)=$ & $52.4( \pm 4.3)=$ \\
\hline
\end{tabular}

* FD Treatment at $10 \mu \mathrm{g} / \mathrm{mL}$.

$¥ ¥ p$ value $<0.01 / ¥ ¥ p$ value $<0.02$ / ¥ $p$ value $<0.05$

no Inh $=$ no significant inhibition observed

Table 3.2 represents those FD that were effective at significantly inhibiting TNF$\alpha$ cytokine production from MC. Out of five separate MC cultures, no activation of cytokine production was observed with compound $48 / 80$ or somatostatin (varying dose 
and incubation times; positive controls using FceRI antibodies) as others have reported [109].

Table 3.2. Mean \% Inhibition of TNF- $\alpha( \pm \mathrm{SD})$ in FD Treated and Untreated MC.

\begin{tabular}{|c|c|c|}
\hline Fullerene Derivative & $A 23187+F^{*}$ & poly-L-lysine $+\mathrm{FD}^{\star}$ \\
\hline Biotin & $83.9( \pm 8.0)^{ \pm}$ & no Inh \\
\hline $\mathrm{C} 70-\mathrm{OH}$ & $60.6( \pm 28.2)=$ & $16.41( \pm 10.5)^{ \pm}$ \\
\hline $\mathrm{CCC}$ & no Inh & no Inh \\
\hline DMAE & $41.1( \pm 12.9)^{ \pm}$ & no Inh \\
\hline Ethanolamine & $52.3( \pm 3.9)$ & no Inh \\
\hline Niacin & $73.2( \pm 9.6)^{=}$ & no Inh \\
\hline NSAID & no Inh & $12.0( \pm 6.2)^{*}$ \\
\hline PC4 & $94.1( \pm 14.2)^{= \pm}$ & no Inh \\
\hline Tetraglutamate & $59.7( \pm 3.0)$ & $48.3( \pm 1.86)^{m}$ \\
\hline Tetrainositol & $95.7( \pm 2.3)^{ \pm}$ & $72.4( \pm 6.7)=$ \\
\hline Tetraphosphate & $39.3( \pm 14.3)^{ \pm}$ & no Inh \\
\hline Tetrapyridine & $51.7( \pm 17.5)^{=}$ & no Inh \\
\hline Tetrasulfonate & $39.9( \pm 2.5)$ & no Inh \\
\hline TGA & $93.7( \pm 4.6)^{2}$ & $70.2( \pm 1.0)=$ \\
\hline TTA & $98.7( \pm 3.6)=$ & $66.8( \pm 3.1)=$ \\
\hline
\end{tabular}

*FD Treatment at $10 \mu \mathrm{g} / \mathrm{mL}$

$¥ ¥ p$ value $<0.01 / ¥ ¥ p$ value $<0.02$ / $p$ value $<0.05$

no Inh $=$ no significant inhibition observed

Calcium ionophore (A23187) is a widely used secretagogue which can directly increase intracellular calcium concentration (calcium) levels to induce mediator release [110]. Nine FD significantly $(\mathrm{p}<0.05)$ inhibited degranulation $(>10 \%)$ (Table 3.1).

Furthermore, 13 FD were capable of inhibiting TNF- $\alpha$ cytokine production $(>40 \%)$ induced by A23187 (Table 3.2). Poly-L-lysine is a small polypeptide of the essential amino acid L-lysine that can induce MC activation $[111,112]$. Seven of the FD inhibited degranulation ( $>10 \%$ ) and six were capable of inhibiting TNF- $\alpha$ production (Table 3.1 
and 3.2). Typical dose response curves of inhibition on degranulation and cytokine production are indicated in Figure 3.1A-D.

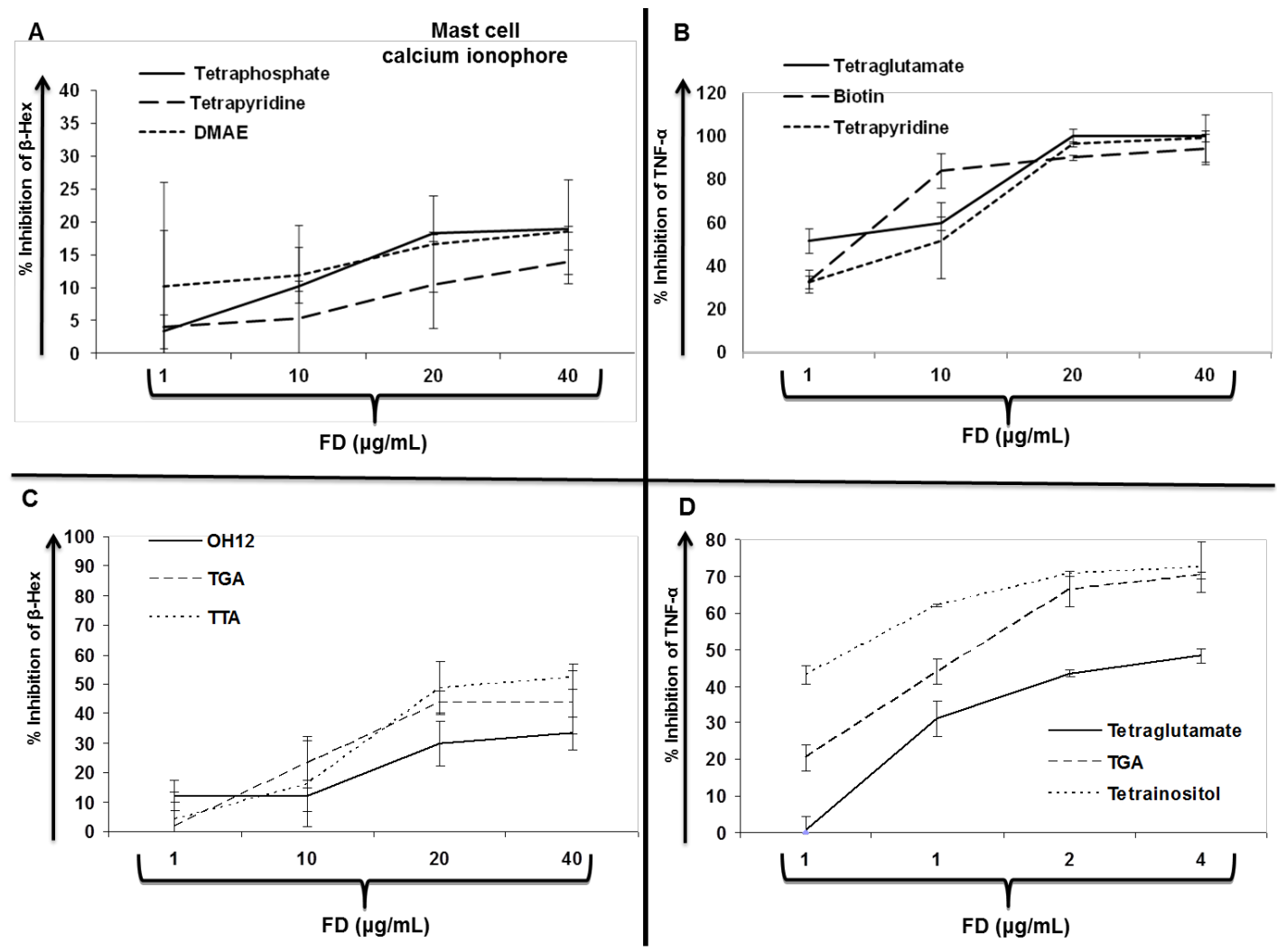

Figure 3.1. Specific FD Inhibit Human MC Calcium-Ionophore and Poly-L-LysineInduced Mediator Release in a Dose Dependent Manner. Mast cells were incubated overnight with FD, washed and activated with increasing concentrations of A23187 (A and B) or poly-L-lysine (C and D) for $30 \mathrm{~min}$ (degranulation, A and C) or $18 \mathrm{~h}$ (cytokine, $B$ and $D$ ). Mediator release was assessed as described in materials and methods. Figures are representative of three separate MC cultures with each condition performed in triplicate.

Compound $48 / 80$ is a polybasic secretagogue, which has previously been shown to activate human $\mathrm{MC}[113,114]$ presumably through direct interactions with guanosine triphosphate (GTP)-binding proteins and MAPK [115]. As seen in Table 3.1, five FD inhibited degranulation in MC challenged with compound 48/80 (>20\%). Somatostatin is 
a peptide hormone that has previously been shown to activate human $\mathrm{MC}[114,116]$ through the G-protein-coupled somatostatin receptor $[114,117]$. Table 3.1 shows a subset of 11 different FD that inhibited degranulation. A representative dose response for inhibition is seen in Figure 3.2A and B.
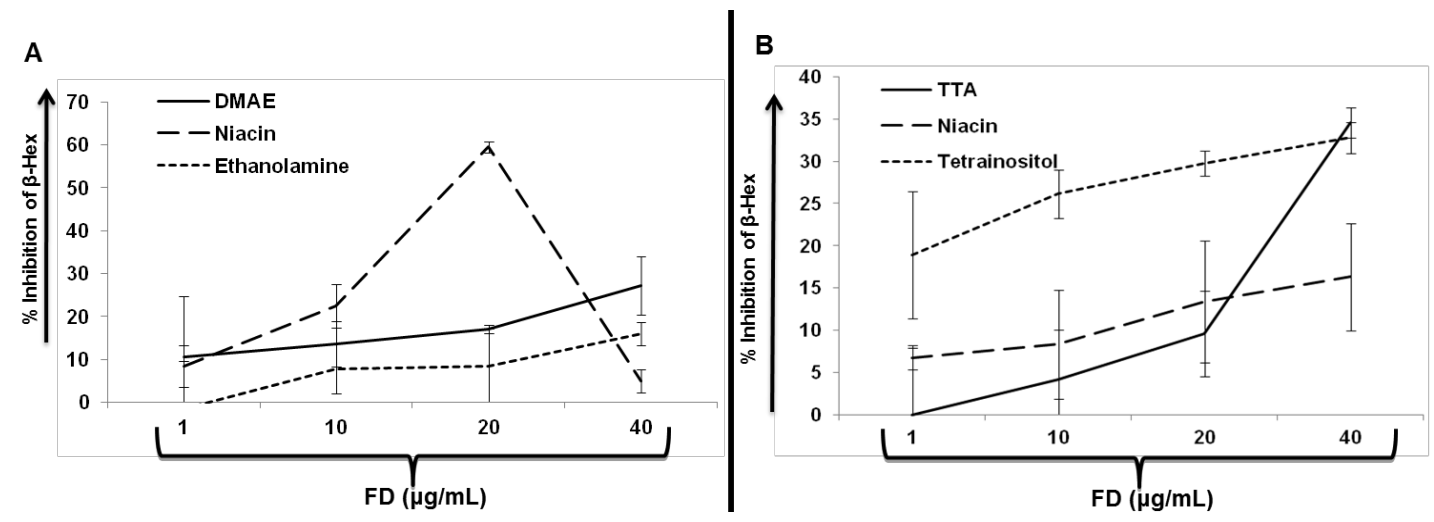

Figure 3.2. Specific FD Inhibit Human MC Compound 48/80- and SomatostatinInduced Degranulation in a Dose Dependent Manner. Mast cells were incubated overnight with FD, washed and activated with increasing concentrations of compound 48/80 (A) or somatostatin (B). Mediator release was assessed as described in materials and methods. Figures are representative of three separate MC cultures with each condition performed in triplicate.

\section{III.3.2 The effect of FD on non-IgE induced mast cell PGD2 release}

The panel of FD (Tables 3.1 and 3.2) evaluated for mediator release were investigated to determine if they could reduce PGD2 production in MC. TGA, Niacin, Biotin, and Tetrainositol were selected based on to their ability to inhibit cytokine production and degranulation in activated $\mathrm{MC}$ in response to a number of stimuli. Neither Biotin nor Tetrainositol resulted in any inhibition of PGD2 production. Mast cells preincubated with TGA or Niacin and challenged with A23187, 48/80, or poly-Llysine decreased PGD2 production by $>60 \%$ in all conditions, (Table 3.3). No detectable levels 
of PGD2 where observed in MC-activated with somatostatin. Additionally, PGD2

production in IgE challenged (FceRI) MC was reduced by nearly $60 \%$ by FD

pretreatment.

Table 3.3. Mean \% Inhibition of PGD2 Production $( \pm \mathrm{SD})$ in FD Treated and Untreated MC.

\begin{tabular}{|l|c|c|c|c|}
\hline \multicolumn{1}{|c|}{ Activator } & TGA & Niacin & Biotin & Tetrainositol \\
\hline A23187 & $79.8( \pm 8.4)$ & no inh & no Inh & no Inh \\
\hline $\mathbf{4 8 / 8 0}$ & no inh & $65.2( \pm 9.8)$ & no inh & no Inh \\
\hline somatostatin & ND & ND & ND & ND \\
\hline poly-L-lysine & $62.1( \pm 12.2)$ & no inh & no Inh & no Inh \\
\hline FcERI & $59.8( \pm 7.8)^{ \pm}$ & no inh & no inh & no Inh \\
\hline
\end{tabular}

* FD Treatment at $10 \mu \mathrm{g} / \mathrm{mL}$.

$¥ p$ value $<0.01 / ¥ p$ value $<0.02 / ¥ p$ value $<0.05$

no Inh $=$ no significant inhibition observed $/ \mathrm{ND}=$ none detected

\section{III.3.3 The effect of FD on non-IgE induced peripheral blood basophil mediator release}

The secretagogues that induce mediator release from PBB are different from those that induce mediator release from MC [118]. The effects of FD on N-formyl-methionineleucine-phenylalanine (fMLP) and poly-L-lysine-induced mediator release from PBB are shown in Table 3.4. Only Ethanolamine inhibited fMLP-induced degranulation (14\%). Tetrainositol, TGA, and Niacin inhibited IL-13 release when activated with fMLP, but none significantly affected poly-L-lysine induced mediator release as seen in Table 3.5. None of the FD evaluated inhibited A23187-induced PBB degranulation (data not shown). 
Table 3.4. Mean \% Inhibition of Degranulation $( \pm \mathrm{SD})$ in FD Treated and Untreated PBB.

\begin{tabular}{|l|l|l|l|l|}
\hline $\begin{array}{l}\text { Fullerene } \\
\text { Derivative }\end{array}$ & $\begin{array}{l}\text { FMLP }(1 \mu \mathrm{M}) \\
+\mathrm{FD}^{*}\end{array}$ & $\begin{array}{l}\text { FMLP }(10 \mu \mathrm{M}) \\
+\mathrm{FD}^{*}\end{array}$ & $\begin{array}{l}\text { poly-L-lysine } \\
(1 \mu \mathrm{M})+\mathrm{FD}^{*}\end{array}$ & $\begin{array}{l}\text { poly-L-lysine } \\
(10 \mu \mathrm{M})+\mathrm{FD}^{*}\end{array}$ \\
\hline Ethanolamine & no Inh & $14.1( \pm 7.2)^{*}$ & no Inh & no Inh \\
\hline Tetrainositol & $49.0( \pm 18.1)^{*}$ & $37.9( \pm 14.1)^{*}$ & $47.8( \pm 5.6)^{ \pm *}$ & $27.7( \pm 1.52)^{*}$ \\
\hline TGA & $22.5( \pm 6.11)^{*}$ & $20.9( \pm 2.7)^{ \pm}$ & $5.7( \pm 1.7)^{*}$ & no Inh \\
\hline Niacin & $8.6( \pm 1.89)^{*}$ & $2.9( \pm 1.98)^{*}$ & $7.5( \pm 2.2)^{*}$ & no Inh \\
\hline
\end{tabular}

* FD Treatment at $10 \mu \mathrm{g} / \mathrm{mL}$.

¥¥ $p$ value $<0.01 / ¥ ¥ p$ value $<0.02 / ¥ p$ value $<0.05$

no Inh $=$ no significant inhibition observed $/ \mathrm{ND}=$ none detected

Table 3.5. Mean \% Inhibition of IL-13 $( \pm$ SD) in FD Treated and Untreated PBB.

\begin{tabular}{|l|c|c|}
\hline \multicolumn{1}{|c|}{ Fullerene Derivative } & FMLP + FD $^{\star}$ & poly-L-lysine + FD $^{\star}$ \\
\hline Ethanolamine & no inh & no Inh \\
\hline Niacin & $86.6( \pm 16.6) \approx$ & no Inh \\
\hline Tetrainositol & $54.8( \pm 19.3)^{\star}$ & no Inh \\
\hline TGA & $83.7( \pm 19.0)^{\star \star}$ & no Inh \\
\hline
\end{tabular}

* FD Treatment at $10 \mu \mathrm{g} / \mathrm{mL}$.

$¥ ¥$ value $<0.01 / ¥ p$ value $<0.02$ / $p$ value $<0.05$

no Inh $=$ no significant inhibition observed

III.3.4 The effect of FD on non-IgE mediated induced ROS activity and intracellular calcium flux in mast cell

We next began to delineate the mechanisms of inhibition focusing on MC given the difficulty in obtaining adequate numbers of PBB for such studies. The activation of $\mathrm{MC}$ and subsequent degranulation is calcium-dependent and results in elevated ROS levels [119]. It was hypothesized that FD reduced MC degranulation by blocking ROS production and calcium responses. Therefore, representative FD was selected based on 
their ability to inhibit A23187, compound 48/80, somatostatin, and poly L-lysine-induced degranulation.

A23187-induced increases in ROS and calcium fluctuations were significantly reduced by TGA ( $\sim 50 \%$ for ROS, $\sim 70 \%$ for calcium) and Ethanolamine ( $\sim 50 \%$ for ROS, $\sim 90 \%$ for calcium) (Figures 3.3A and 3.4A). Niacin and Tetrasulfonate significantly reduced compound 48/80-induced ROS activity ( $>30 \%$; Figure 3.3B), but selectively effected calcium levels (Niacin $>80 \%$ inhibition and Tetrasulfonate was ineffective; Figure 3.4B). Somatostatin stimulated MC pretreated with Biotin and TTA inhibited ROS and calcium activity by approximately $30 \%$ (ROS) and 75\% (calcium) (Figures 3.3C \& 3.4C). Lastly, Figures 3.3D and 3.4D demonstrate TGA and TTA blunted ROS generation $>50 \%$ in response to poly-Llysine as well as inhibited calcium approximately $30 \%$ compared to the positive control. Thus, FD appears to inhibit mediator release through the blunting of secretagogue-induced cellular increases in ROS and intracellular calcium stores. 


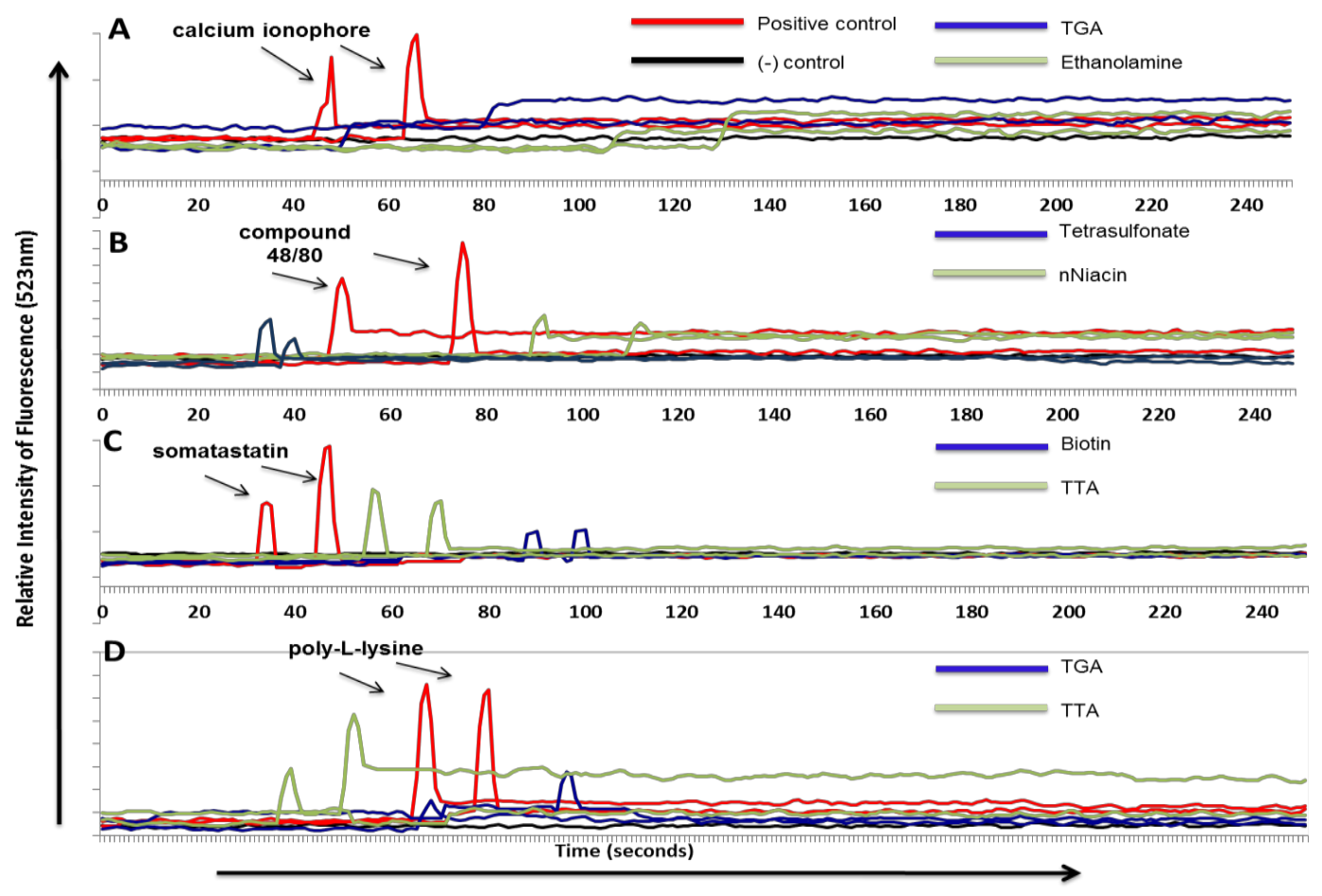

Figure 3.3. Fullerene Derivatives Inhibit Secretagogue-Induced Elevations in Intracellular ROS Levels. Mast cells were incubated overnight with FD, washed and DCF-DA added to cells for $30 \mathrm{~min}$ at $37^{\circ} \mathrm{C}$. After washing cells were activated with optimal concentrations of the indicated secretagogue and the fluorescence intensity measured at $525 \mathrm{~nm}$ after establishing baseline. Figures show duplicate samples for each condition and are representative of three separate MC cultures. All positive controls (activated but not pre-incubated with FD) were represented by red traces and all negative controls (non-activated and non-preincubated with FD) are denoted by black traces. 


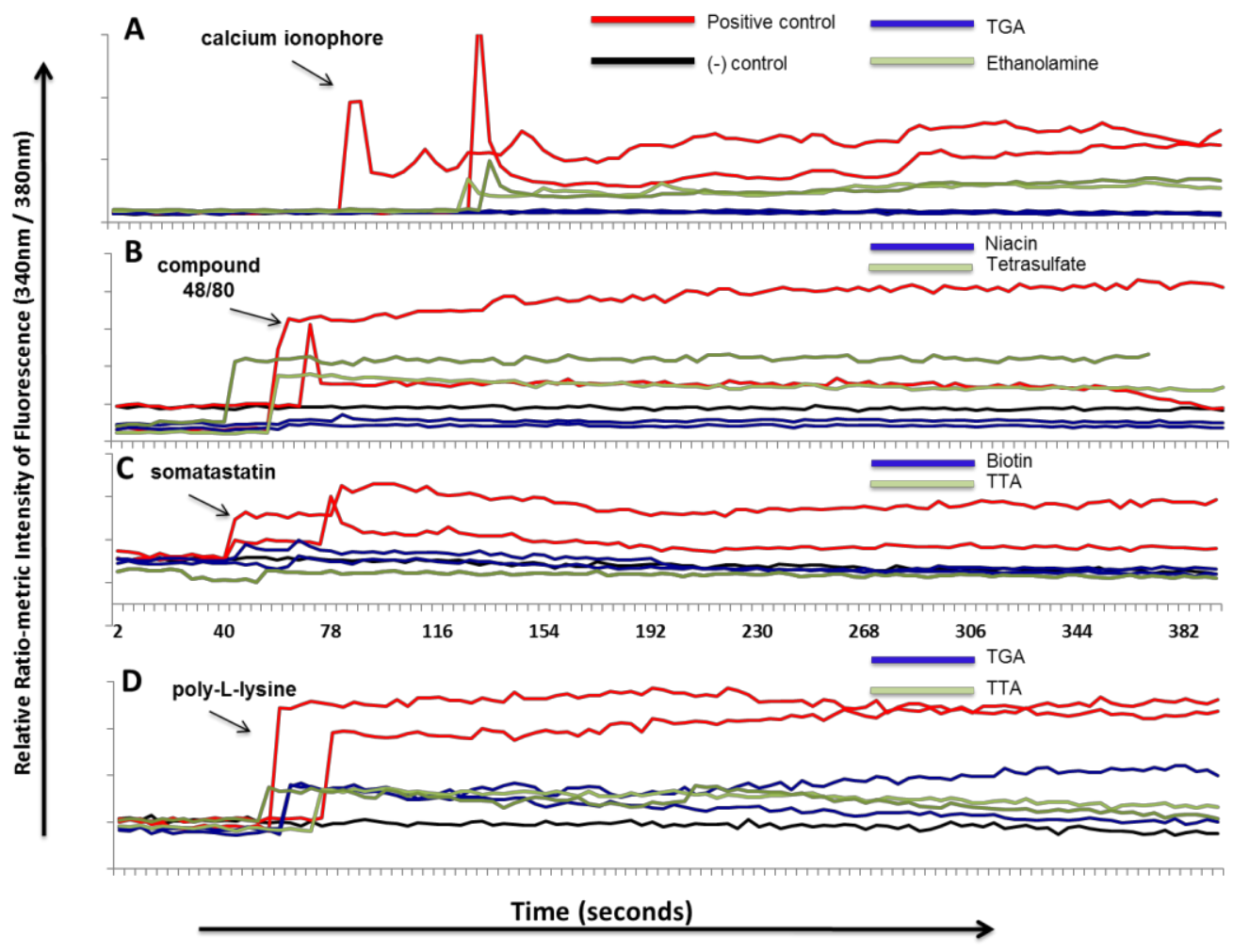

Figure 3.4. Fullerene Derivatives Inhibit Secretagogue-Induced Elevations in Intracellular Calcium Levels. Mast cells were incubated overnight with FD, washed and FURA 2 added to MC and incubated for $20 \mathrm{~min}$ at $37^{\circ} \mathrm{C}$ in the dark. After washing cells were activated with optimal concentrations of the indicated secretagogue and intensity of fluorescence was read as a ratio $340 \mathrm{~nm}$ and $380 \mathrm{~nm}$ wavelengths. Figures show duplicate samples for each condition and are representative of three separate MC cultures.

\section{III.3.5 The effect of FD on non-IgE mediated signaling pathway intermediates}

To further delineate the mechanism of action of the FD on non-IgE-mediated degranulation we examined Western blotting using antibodies to the phosphorylationdependent activated forms of LAT and ERK1/ERK2 [120, 121]. As seen in Figure 3.5A neither Biotin or TTA significantly affected A23187-induced changes in MAPK or LAT. Niacin and Tetrasulfatonate both reduced compound 48/80-induced phospho-activation 
of MAPK and LAT (Figure 3.5B) while Biotin and TTA both reduced somatostatininduced changes (Figure 3.5C). Both TGA and TTA reduced poly-L-lysine induced activation of MAPK with minimal effects on LAT. No changes in the phosphorylation state of other signaling pathways examined including the Src family of kinases and phosphoinositol 3-kinase (PI3K) pathway were observed under these conditions (data not shown).

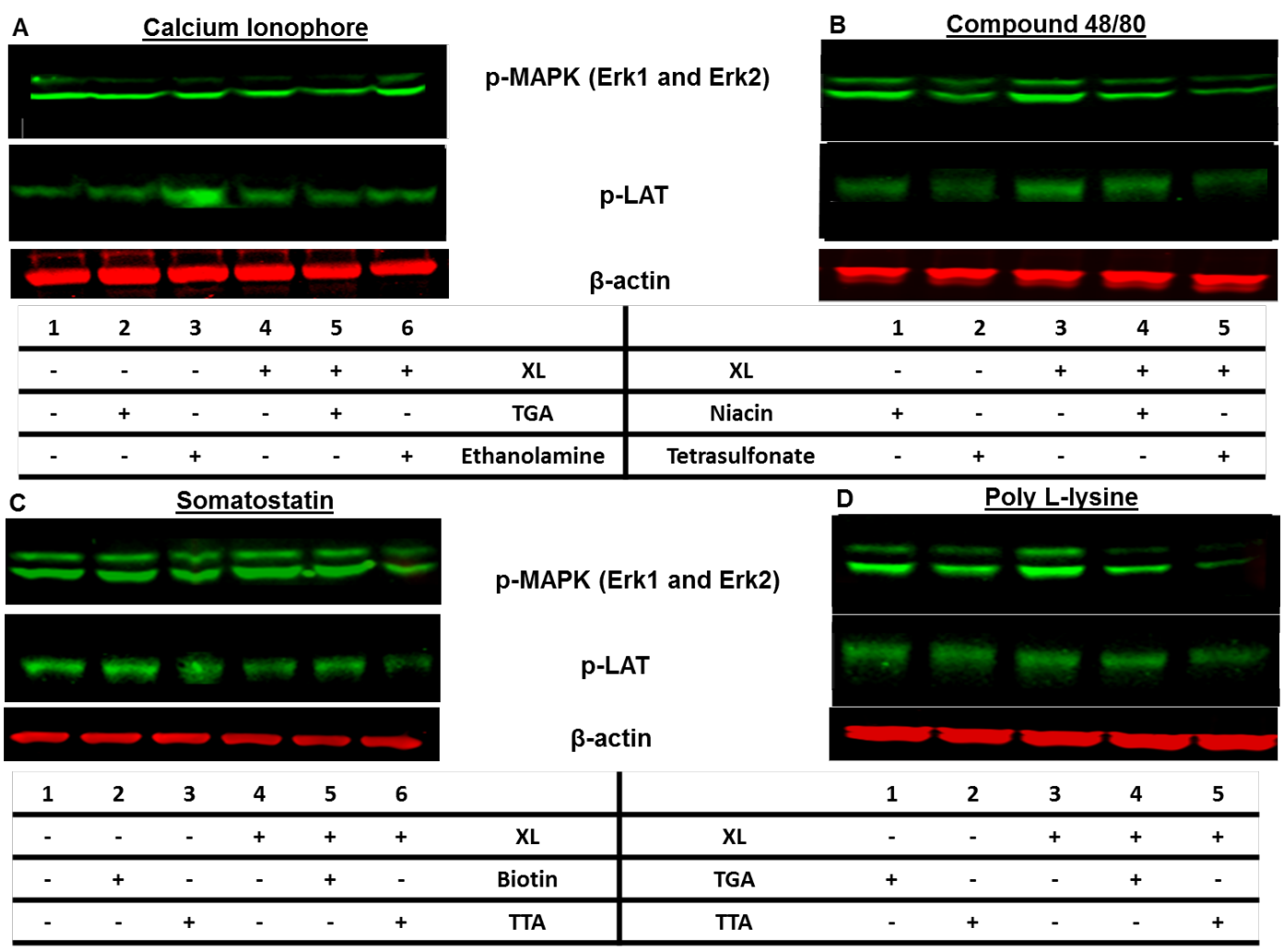

Figure 3.5. Inhibition of Mediator Release in Calcium Ionophore, Compound 48/80, Poly-L-Lysine, and Somatostatin Challenged MC Involves the Down-Regulation of MAPK and LAT Phosphorylation. MC were incubated with or without the FD overnight, washed and activated with the indicated secretagogue. Western blotting was performed as described previously in Methods with the indicated Abs and Odyssey IR800-anti-rabbit secondary antibodies. In both cases, the total protein content in lysates was measured with the house keeping gene $\beta$-Actin and Odyssey IR680-anti-mouse secondary antibody. 


\section{III.4 Conclusions}

Mast cells and PBB contribute to several disease processes through the release of inflammatory mediators through both $\operatorname{IgE}$ and non-IgE mechanisms. Thus, therapies aimed at stabilizing them and preventing the release of their mediators has been the subject of research for many years. For example, omalizumab, the humanized IgE specific IgG1 monoclonal antibody, which limits the amount of free IgE available to bind FceRI on the surface of MC/PBB, can control severe allergic asthma [122, 123]. Another MC stabilizer, Cromolyn, has been effectively used to treat a wide range of MC-driven diseases [124-126]. The significance of these studies is that FD has a broad range of inhibitory capabilities toward inflammatory cells and has not demonstrated acute toxicity in vitro. Since $\mathrm{MC} / \mathrm{PBB}$ can be induced to secrete their inflammatory mediators through several non-overlapping pathways these molecules represent a new strategy for therapeutics aimed at those disease mediated by these cells such as allergy, asthma, and arthritis.

As in our previous studies examining IgE-FceRI-mediated degranulation and cytokine production $[20,22]$, the efficacy in of FD was strongly dependent upon specific side chains additions; approximately $20 \%$ of the FD tested exhibited significant inhibition. In addition, certain secretagogues were unaffected by FD preincubation, indicating that the pathway in which the stimuli activates the cell is critical to FD efficacy. That is, the FD does not result in a blanket protection of the cells response to all stimuli, but the FD specifically regulates specific signaling pathways. Mast cells and PBB responded differently to fullerene preincubation, suggesting that the signal transduction 
pathways leading to mediator release are dissimilar as has been previously reported [127]. Further variation in how FD affected cell responses was demonstrated in the type of mediator release (degranulation, cytokine, prostaglandin production, or a combination thereof). Our strategy, including these studies, is to identify those FD that stabilize both IgE and non-IgE pathways and pursue more in depth toxicity, pharmacokinetic, and biodistribution studies. To this end, TGA is currently a top candidate demonstrating no toxicity in vitro and in vivo $[22,49]$, is a potent human MC/PBB stabilizer to FceRI dependent stimuli [22], prevents and reverses MC-dependent asthma in mouse models [32], and can inhibit non-FceRI responses as demonstrated herein.

There were no clear structure-activity relationships that were established from these studies. Particular FD was more effective controllers of numerous stimuli, for example Tetrasulfonate showed statistically significant reductions in degranulation regardless of stimulant used. However, compounds such as $\mathrm{CCC}$ were only successful at blunting somatostatin-induced degranulation, while it did not have any effect on the mediator release elicited by any of the other compounds. Mechanistically, these compounds significantly prevented activation of MAPK and LAT suggesting they either block the signaling intermediates directly or some other intermediate upstream. The inhibition of mediator release by FD was paralleled by reductions in secretagogueinduced elevations in ROS/calcium levels as well as the signaling intermediates MAPK and LAT. These findings are similar to those previously demonstrated when examining ROS/calcium levels and phosphorylation-of signaling intermediates in FceRI-challenged MC $[20,22]$. Current studies are aimed at identifying potential intracellular binding 
partners of FD similar to experiments performed to identify binding partners in response to FceRI stimulus [128].

Another example in our attempts to rationally designed FD for specific disease involves the role of $\mathrm{MC}$ in atherosclerosis. A link between $\mathrm{MC}$ activation and atherosclerosis has now been clearly demonstrated $[103,129]$ as their numbers are greatly increased in the intima at sites of arterial plaque rupture [129], in advanced plaque lesions in the carotid artery [130], and patients who died of acute myocardial infarction have an increased number of degranulated MCs at the actual site of plaque erosion or rupture [131]. Therapies aimed at treating atherosclerosis include niacin which blocks vascular inflammation, ROS, and inflammatory cytokine production in conjunction with diminishing NF-KB activation [132]. Thus, the niacin FD was constructed and demonstrated inhibition of mediator release from several non-IgE pathways through reduced cellular ROS and ERK1/2 phosphoactivation. Mast cell-stabilizing therapies such as Cromolyn have shown promise in ApoE models of atherosclerosis (the classic animal model for atherosclerosis) where it prevents intra-plaque hemorrhage [133]. Thus, strategies aimed at blocking MC activation before it occurs could represent a new strategy for treating atherosclerosis.

Prostaglandin D2 is produced by MC and recruits Th2 cells, eosinophils, and PBB and it is critical to development of allergic diseases such as asthma [24]. In mammalian organs, large amounts of PGD2 are found only in the brain and in MC. Previous studies found MC-produced PGD2 is the primary mediator of vasodilatation (the "niacin flush") after ingestion of niacin (nicotinic acid) [41]. Previously reported data reveals that non- 
IgE induced PGD2 production was attenuated when ROS production was reduced [134]. This may be in part due to the mechanism that ROS facilitates the formation of a hydrogen bond necessary for PGD synthase activation [134]. In our study, the FD evaluated that was effective at reducing ROS generation in $\mathrm{MC}$ was also effective regulators of PGD2 production, possibly under the proposed mechanism of modulating PGD synthase activity.

In conclusion, these studies further delineate the ability of FD to affect human $\mathrm{MC}$ and PBB responses so that diseases mediated by these cell types may be a target for FD-derived therapies. It is demonstrated that certain FD can differentially modulate mediator release from $\mathrm{MC}$ and $\mathrm{PBB}$ in response to a variety of secretagogues. This inhibition can involve the blunting of activation-induced increases in ROS, the release of intracellular stores of calcium, and the phospho-activation of MAPK and LAT. Furthermore, the inhibition is strictly governed by several factors, the degree and type of functionalization of the fullerene nanomaterials, the type of cells that are analyzed, the mediator being evaluated, and how the cell is being activated to illicit the mediator release. These studies further extend the utility of FD as inhibitors of MC mediator release and cytokine production through non-IgE mediated pathways. 


\section{CHAPTER IV}

\section{FUNCTIONALIZED GADOLINIUM ENDOFULLERENES DETECT ATHEROSCLERTOIC PLAQUE LESIONS}

\section{IV.1 Introduction}

Atherosclerotic cardiovascular disease results in close to 20 million deaths annually. A hallmark of the disease is the accumulation of plaque lesions in blood vessel walls which can rupture and result in acute myocardial infarction and stroke. Clearly better diagnostic tools are needed to identify incipient disease, monitor disease progression, and pinpoint factors that predict catastrophic ruptures.

At present, it is difficult for physicians to specifically detect and quantify plaque lesion buildup in vessel walls. Cardiovascular magnetic resonance (CMR) is one of several techniques being investigated to identify plaque burden in patients so that interventions can be conducted before rupture occurs $[135,136]$. We have used novel gadolinium (Gd)-containing $\mathrm{C}_{80}$ endohedrals Trimetaspheres ${ }^{\mathrm{TM}}, \mathrm{TMS}, \mathrm{Gd}_{3} \mathrm{~N} @ \mathrm{C}_{80}$ ) [137] as a platform to develop new atherosclerotic targeting contrast agents (ATCA) for CMR. The TMS-based molecules have 25 fold increased relaxivity compared to other contrast agents, reduced risk of metal toxicity, and can be customized to address issues surrounding solubility, specificity, etc. [71]. TMS were functionalized with highly specific ligands for the scavenger receptor CD36 found on the surface of macrophage foam cells in plaque lesions [79]. It is demonstrated that ATCA specifically bind to and are taken up within foam cells in vitro and are able to detect lesions in plaque-susceptible 
mice in vivo (Apolipoprotein E deficient mice [ApoE -/-]). Thus, TMS can be targeted to specific biomarkers in sufficient quantities to provide enhanced imaging of atherosclerotic plaque under physiological conditions. These results suggest that the ATCA may be a new tool for detecting atherosclerotic plaque.

\section{IV.2 Methods}

\section{IV.2.1 ATCA synthesis, functionalization, and characterization}

The method to synthesize the TMS uses an electric-arc process to encapsulate Gd within a carbonaceous cage, $\mathrm{C}_{80}$ [137], which was used as the starting material for the ATCA. The molecular weight of TMS (1,446 Da) was determined using matrix-assisted laser desorption/ionization (MALDI) with a time-of-flight (TOF) mass spectrometer. Elemental analysis determined that TMS contains $10.15 \pm 0.25 \mathrm{mM}$ Gd which approximates the value estimated from the molecular weight. No free Gd (Gd outside the carbon cage) was detected using an Arsenazo III colorimetric test [138] and ICP analysis

of liquid after dialysis of the TMS. Further, atomic force microscopy (AFM) measurement of the TMS showed the majority of the nanoparticles are 1.1-1.3 nm in height with some particles approaching $3.0 \mathrm{~nm}$ to $4.0 \mathrm{~nm}$, suggesting minimal agglomeration and/or aggregation.

The ATCA was developed as shown in Appendix A in which the TMS is functionalized with hydrophilic and lipophilic groups [71] and incorporated into liposomes [48]. The resulting liposome-TMS were formulated with or without CD36 
ligands intercalated within the liposome bilayer membrane $[79,139]$. The ATCA was characterized as seen in Appendix B.

\section{IV.2.2 Cell culture}

Foam cells were generated using oxidized-low density lipoproteins (oxLDL) from human plasma as described previously [140] and verified using oil red-o (ORO) staining [141]. The upregulation of CD36 expression in foam cells was confirmed using both PCR and FACs analysis with CD36-specific antibodies. As a control, the non CD36expressing cell line 3 T3-F442A pre-adipocytes were used to detect non-specific binding. The U937 cells and foam cells were examined for toxicity using up to $100 \mu \mathrm{g} / \mathrm{ml}$ (five times the amount used for in vivo studies) for 10 days. No significant differences in cell viability was observed with ATCA-treated cells compared to controls (not shown) which further supports the observation that derivatized fullerenes are not toxic in vitro $[22,49]$. Cells were treated with CD36-targeted or non-targeted controls at various concentrations for $24 \mathrm{~h}$, centrifuged and the supernatant saved for Gd analysis. After a quick wash the cell pellet was disrupted by sonication and supernatant and cell pellet subjected to ICP analysis or neutron bombardment (both methods gave similar results; not shown) to determine Gd concentration as described below.

\section{IV.2.3 Cell uptake of the ATCA revealed by FIB-SEM crossbeam microscopy}

In order to examine cell uptake of the ATCA we used a Zeiss FIB-SEM

crossbeam workstation. Foam cells were incubated with or without ATCA or nontargeted control $(20 \mu \mathrm{g} / \mathrm{ml})$ for various times $(16,36,72 \mathrm{~h})$. As a control non-targeted 
ATCA were incubated for the same times. In addition, non-foam cells were incubated with ATCA for $24 \mathrm{~h}$. Cells were washed and fixed using 1\% glutaraldehyde solution and 0.1 M cacodylate buffer for $2.5 \mathrm{hrs}$ at room temperature. Samples were mounted on a microscope slide covered in aluminum foil and subjected to graded EtOH incubations (60\%-100\%/10 min each) and stored in a desiccated environment until microscopic analysis.

The mounted samples were put in the FIB-SEM crossbeam workstation, while a focused ion beam (FIB) was used to cut the cells and expose inner structure of the cell. Simultaneously, scanning electron microscope (SEM) was used to image the cross section milled via FIB, this is capable because both the ion beam and electron beam can focus on the exactly same area. The FIB was operated at $30 \mathrm{kV}, 20 \mathrm{pA}$ and SEM was operated at $1 \mathrm{kV}$ to reduce potential beam charge and cell damage. A secondary electron detector was used to reveal morphology and energy. Additionally, the energy and angle selective backscattered electron (EsB) detector was applied to detect ATCA existence and spatial distribution with nanometer level resolution inside the cells, since heavy element Gd-containing $\mathrm{C}_{80}$ endohedrals will express themselves much brighter in EsB images if TMS exists.

\section{IV.2.4 Western blotting and quantification of CD36-specific phosphor-signaling}

\section{intermediates}

To confirm the specificity of CD36 ligand binding on the ATCA to the CD36 receptor and examine the mechanisms underlying the interactions between the ATCA and 
foam cells, Western blotting was used as described previously [108]. Following ATCA challenge, foam cells were lysed directly in boiling denaturing sample buffer consisting of tris-buffered saline with triton-X-100 (0.5\%) and protease inhibitors. Proteins were separated on a $10 \%$ gels in tris-glycine SDS running buffer. Western blotting and image quantification was performed using the Odyssey Imaging System (LI-COR Biosciences). Band intensities were captured using the Odyssey scanner and bands quantified by measuring the number of pixels in each band using a box drawn for the same area of measurement for each separate blot. Band intensities were normalized for loading by dividing the number of pixels in each band with the housekeeping band intensity ( $\beta$-actin) on the same blot.

\section{IV.2.5 Flow cytometry}

In order to confirm the upregulation of CD36 expression on foam cells, U937 cells (negative control) or PMA/oxLDL-transformed cells (foam cells) were washed with $\mathrm{PBS} / 1 \% \mathrm{BSA}$, and blocked for $30 \mathrm{~min}$ at $4^{\circ} \mathrm{C}$ with a $1 / 500$ dilution of normal human serum. The cells were washed and incubated with FITC-labeled anti-CD36-FITC, or FITC-labeled isotype control (BD Biosciences; $10 \mu \mathrm{g} / \mathrm{mL}$ ) for one hr. at $4^{\circ} \mathrm{C}$. After three washes, cells were resuspended in $400 \mu \mathrm{L}$ of PBS. The mean intensity of fluorescence was determined for at least 10,000 cells using a FACScan flow cytometer (BD Biosciences). All experiments were performed in duplicate. 
IV.2.6 CMR study

The lesions in arteries of ApoE -/- mice progress from fatty streaks to foam cellcontaining plaque in a similar way as humans $[80,142]$. ApoE $-/-$ mice $(23$ weeks; female) and non-diseased controls were anesthetized initially with isoflurane (3\%) and oxygen $(3 \mathrm{~L} / \mathrm{min})$ in an induction chamber and kept under constant sedation via a nose cone while monitoring vital statistics as described [143]. CMR was performed blinded by personnel with no knowledge of targeted and non-targeted compounds. All animal studies were approved by Wake Forest School of Medicine Animal Care and Use Committee.

\section{IV.2.7 Signal enhancement measurement}

Images acquired after the ATCA injection were examined for areas of hyperintensity in the aorta wall (selected at random). As a comparison whole aortas were analyzed. Regions appearing hyper-intense after ATCA injection were manually traced using ImageJ (nih.org). The contrast to noise ratio was calculated using a reference region of interest (ROI) next to the plaque lesion or of the whole aorta. The same regions were traced in the pre-ATCA injection image and in all the post-ATCA time point images. The contrast to noise was calculated using the same reference ROI and then the contrast-to-noise ratio (CNR) values were normalized to the pre-CA CNR value. The quantification of signal intensities were tabulated and analyzed for all variables and expressed as mean \pm standard error (SE) from five separate animals per group (Figure 4.1B and Figure 4.1C). The StatPlus (Analyst Soft, 2011) software was used to evaluate a two (group) $\times$ two (time) analysis of variance $($ ANOVA) with repeated measures was 
evaluated to analyze data. Tukey LSD post hoc tests were used to examine pair-wise differences. Significance was set at $\mathrm{p}<0.05$.

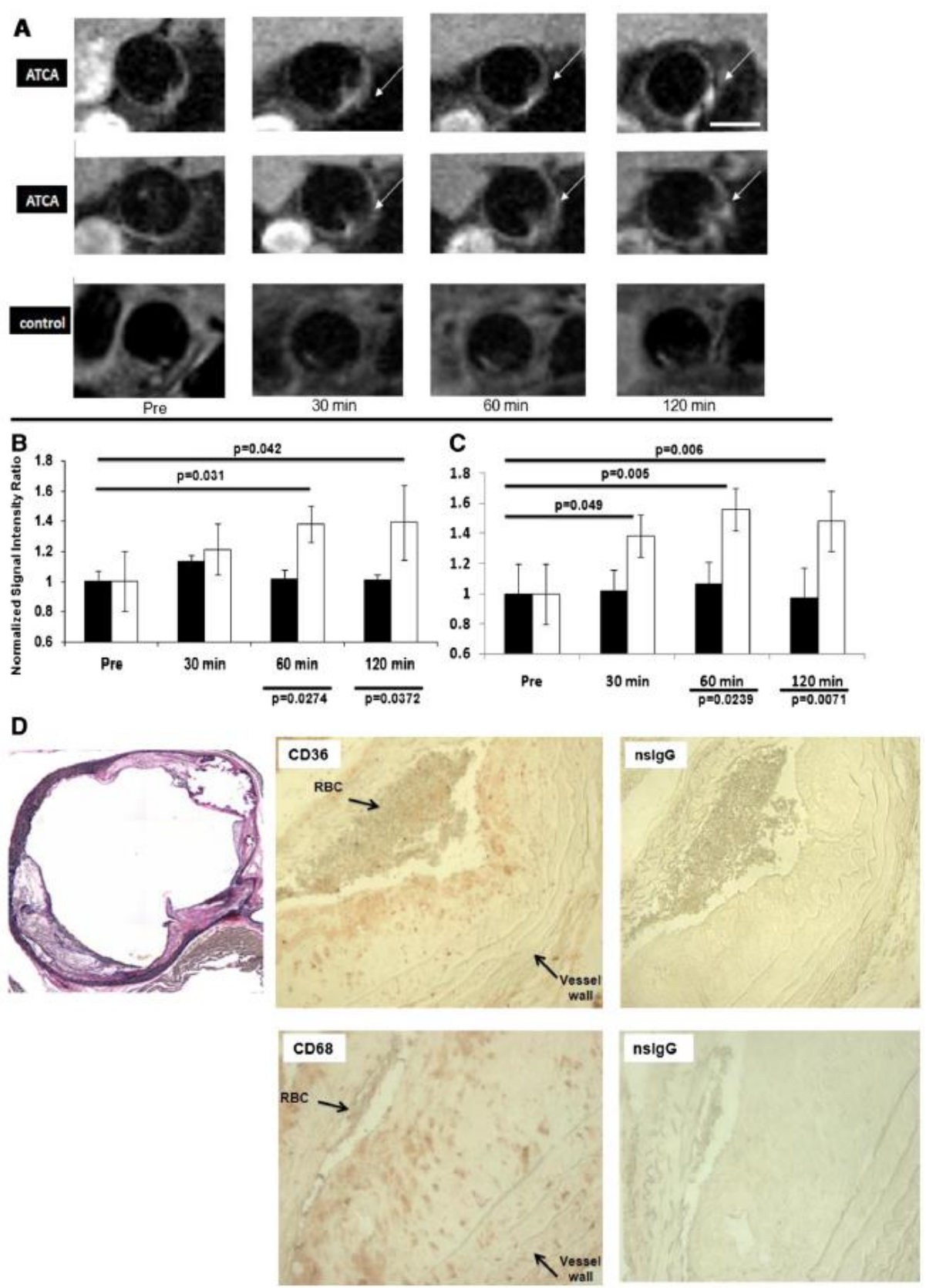

Figure 4.1. ATCA can Detect Inflammatory Plaque In Vivo. ApoE-/- mice (23 wks; $\mathrm{n}=5 / \mathrm{grp})$ were injected i.v. $(100 \mu \mathrm{g} / 100 \mu \mathrm{l})$ with ATCA (top and middle) or non- 
targeted control (bottom) and images acquired at the indicated times. Bar $=\mathbf{1 . 0} \mathbf{~ m m}$. Arrows indicate contrast agent binding to the plaque. A. Representative images from two (ATCA) or one (control) different mice injected with ATCA. B, C.

Quantification of signal enhancement of ATCA. In B, the brightest voxels (approximately $>10$ spots/section) in the aorta wall from 5 separate animals were measured excluding artifact. The ratio of the Signal Intensity (SI) of the brightest voxel in the aorta wall to the SI of the (non-affected) myocardium for each time point was calculated. In $\mathrm{C}$, the ratio of SI of the whole region lining the aorta wall to the SI of the (non-affected) myocardium was measured and the average signal intensity was recorded for 5 separate slices from each time-point. The SIenhanced/SI-myo ratio from each time point to its pre scan SI-enhanced/SI-myo ratio were normalized (prescan values $=1$ ). Data is expressed as mean of $5 \mathrm{CMR}$ slices \pm SD $(n=5$; animals/grp; * indicates significance $(\mathbf{p}<0.05)$. D. Plaque lesions express macrophages and CD36 receptors. Aortas were stained with H\&E (left), rat anti-mouse CD36 (middle), rat anti-mouse CD68 (bottom) or non-specific rat IgG (nsIgG). Peroxidase-conjugated anti-rat Abs were added and developed using AEC. The brown staining represents CD36- or CD68-positive cells located in the plaque lesions. Results are representative of at least three separate aortas.

\section{IV.2.8 Histology, immunohistochemistry, and Gd detection in tissue}

The aorta of each animal was removed and fixed in Tissue-Tek OCT (Miles, Elkhart, IN) and frozen with liquid nitrogen. The tissue was sectioned onto siloconized slides and histopathology assessed using hematoxylin for assessing plaque accumulation $[144,145]$. To examine CD36 and CD68 expression on macrophages in the plaque lesions the tissue sections were fixed in Carnoys, embedded in parafilm, and tissue sections placed on microscopic slides and probed with antibodies to CD36 and CD68 (both from Serotec) followed by horseradishconjugated secondary antibodies and developed using AEC as described previously [29, 146]. As a control nonspecific, isotype-matched control antibodies (for CD36 and CD68) were used. Images were captured using a camera-equipped Zeiss AXIO Observer. For tissue distribution of Gd, organs (or ATCA sample used to inject to determine total Gd counts) were weighed and 
placed in pyrex tubes and combusted to ash at $900^{\circ} \mathrm{C}$ for $1 \mathrm{~h}$. The samples were again weighed and resuspended in $1.0 \mathrm{ml}$ of $12 \mathrm{M}(37 \%) \mathrm{HCl}$. The Gd was measured using ICP and percent Gd in tissue calculated based on the total amount of Gd in the ATCA sample used for injections.

\section{IV.2.9 Toxicological evaluation}

ApoE -/- were injected i.v. with PBS or $1,000 \mu \mathrm{g} / 100 \mu \mathrm{l}(10$ times more than optimized for imaging studies) of ATCA. Mice (four/group) were sacrificed at days two, seven, and 14 and alanine aminotranferease (ALT) and aspartate aminotransferase (AST) levels evaluated in serum. The ALT and AST are transaminase enzymes that leak out into the general circulation when the liver is injured. In separate experiments, ATCA were injected as above, tissue harvested at day seven, and subjected to neutron bombardment or ICP for Gd quantification. An aliquot of the ATCA (not injected) was measured separately to determine the percentage cleared from the mice.

\section{IV.3 Results}

IV.3.1 Plaque targeting contrast agents are selectively taken up by foam cells through CD36 receptor

Atherosclerotic plaque have higher numbers of macrophage foam cells that express CD36 scavenger receptors on their cell surface [147]. Given that the CD36 actively uptake extracellular lipids into their cytoplasmic membrane [148], we hypothesized that this may be a way to incorporate the ATCA contrast agent into foam 
cells in sufficient enough quantities that CMR could be performed. Foam cells were induced using PMA/oxLDL and the upregulation of CD36 expression was confirmed using FACs analysis (Figure 4.2A). As seen in Figure 4.2B, ATCA had a significant uptake into the CD36-expressing foam cells. The control compound for ATCA without the CD36 targeting moiety ligands (control) was not taken up within the cells suggesting the CD36 receptor was responsible for the uptake of the compounds within the cells. In data not shown, there was no uptake in non-foam cell monocytes or non-monocytic, tissue cells (3T3).

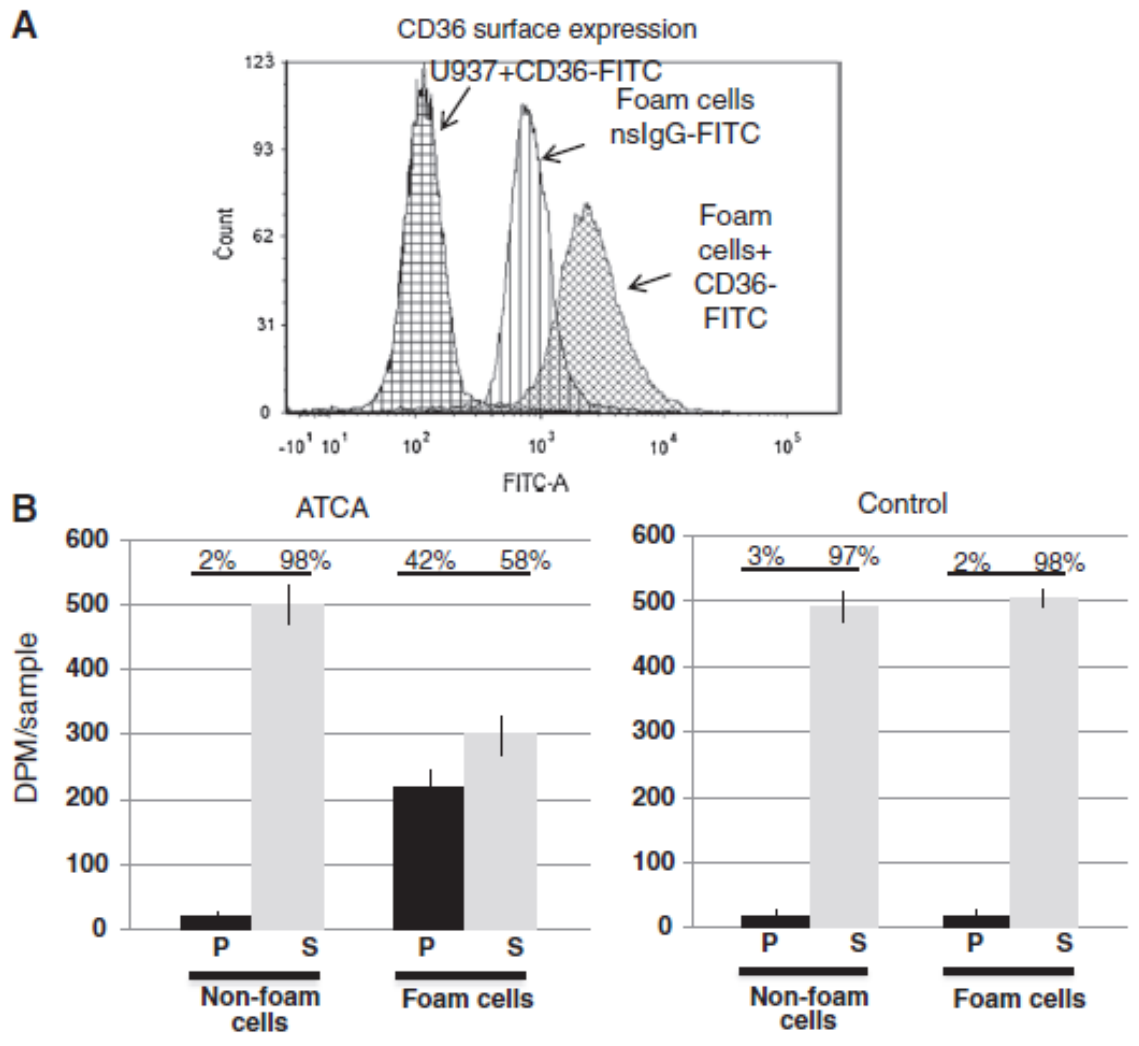

Figure 4.2 - Upregulation of CD36 Receptors on Foam Cells. A. U937 cells (in duplicate) were treated as described in Methods, washed and incubated with antibodies to CD36 or an isotype matched control. The graph shows the average 
mean fluorescent intensity (MFI) of the signal $( \pm \mathrm{SD})$ and is representative of three separate experiments. B. Testing ATCA for foam cell binding. U937 cells (non-foam) were converted into foam cells (foam) using oxLDL and PMA as described. Cells were washed and ATCA $(10 \mu \mathrm{g} / \mathrm{ml})$ added overnight in a $\mathrm{CO}_{2}$ incubator. The next day cells were washed with media and the supernatants $(S)$ and pellets $(P)$ subjected to ICP for Gd detection. The percentage of $\mathrm{Gd}$ in the pellet and supernatant was calculated based on the total amount added to the cells.

We next used a FIB-SEM to follow the ATCA inside foam cells and determine the length of time it remains. Figure 4.3A-3H demonstrates that fullerene-based compounds can be detected inside human foam cells. The ATCA is shown to be more scattered inside the cell at earlier time points (Figure 4.B, D) and begins to aggregate with lesser amounts inside the cell at later time points (Figure 4.F) suggesting cell excretion. The non-targeted control was not detected inside the foam cells (Figure 4.G, H) further suggesting the specificity of the ATCA for foam cells. 


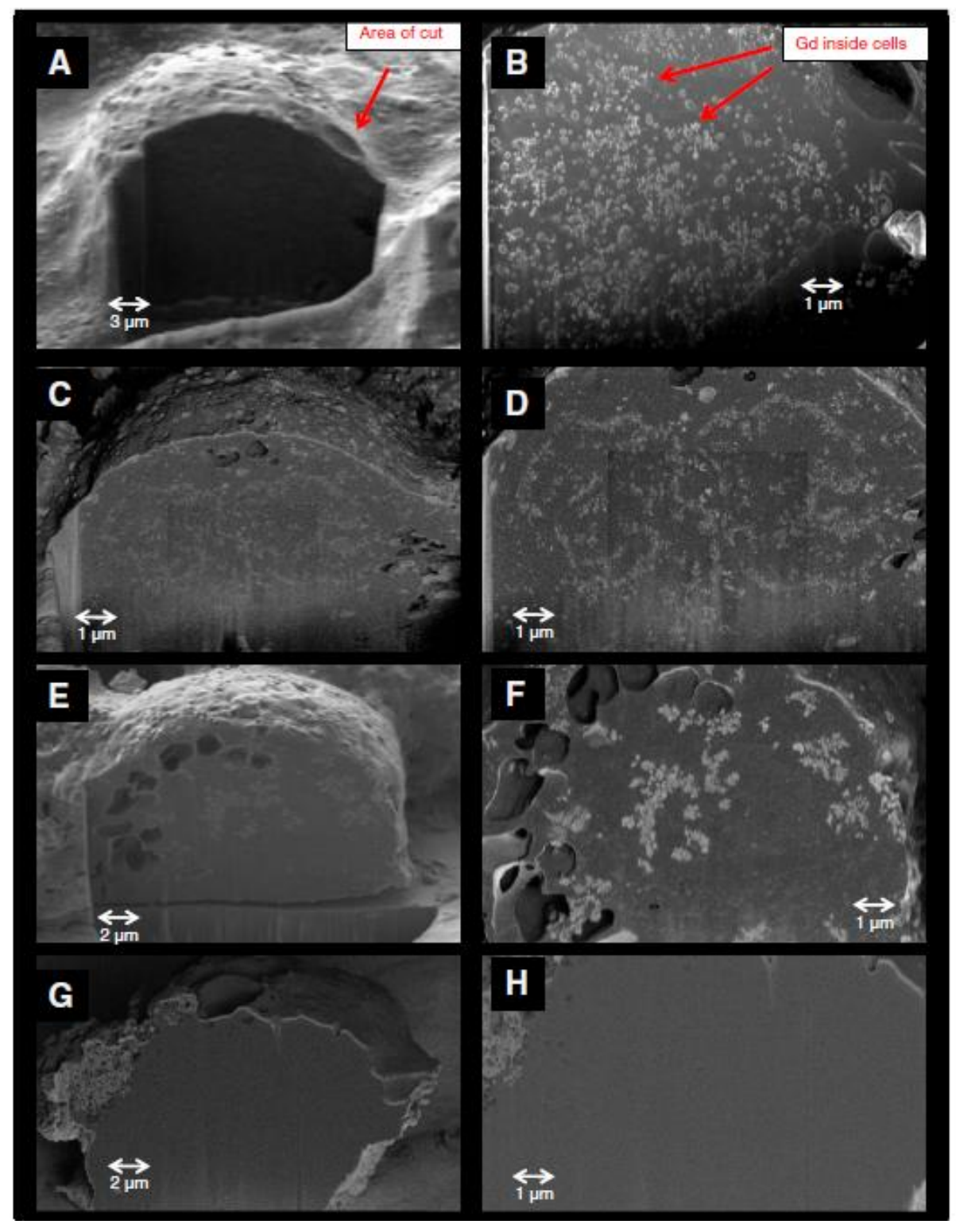

Figure 4.3. ATCA are Taken Up by Foam Cells. Foam cells were incubated with ATCA $(10 \mu \mathrm{g} / \mathrm{ml} ; A-F)$ or non-targeted control $(\mathrm{G}, \mathrm{H})$ for $16(\mathrm{~A}, \mathrm{~B}), 48(\mathrm{C}, \mathrm{D})$ or 72 (E,F) hrs, washed, fixed, and subjected to FIB-SEM. Left columns (A,C,E,G) shows the morphology images taken by secondary electron detector after focused ion beam cut and the right column $(\mathrm{B}, \mathrm{D}, \mathrm{F}, \mathrm{H})$ shows the corresponding material contrast images taken by energy and angle selective backscattered electron (EsB) detector for the same region. The foam cell-targeted ATCA are clearly revealed inside the cells but not in the non-targeted controls. In parallel, an aliquot of cells were used to measure cell viability by trypan blue exclusion and revealed no cell death induced by ATCA addition compared to non-treated controls (not shown). 
To definitively establish the specificity of ATCA binding to the CD36 receptor we used Western blotting and quantification of CD36-associated signaling molecules induced by ATCA treatment. Previous studies have shown that Erk, Lyn, and JNK2 are activated by the binding of oxLDL to CD36 receptors on macrophages $[149,150]$. When foam cells were challenged with ATCA there was a dose (Figure 4.4A) and time (Figure 4.4B) dependent activation of these signaling molecules. This provides definitive evidence that ATCA specifically target foam cells through CD36 receptors. 


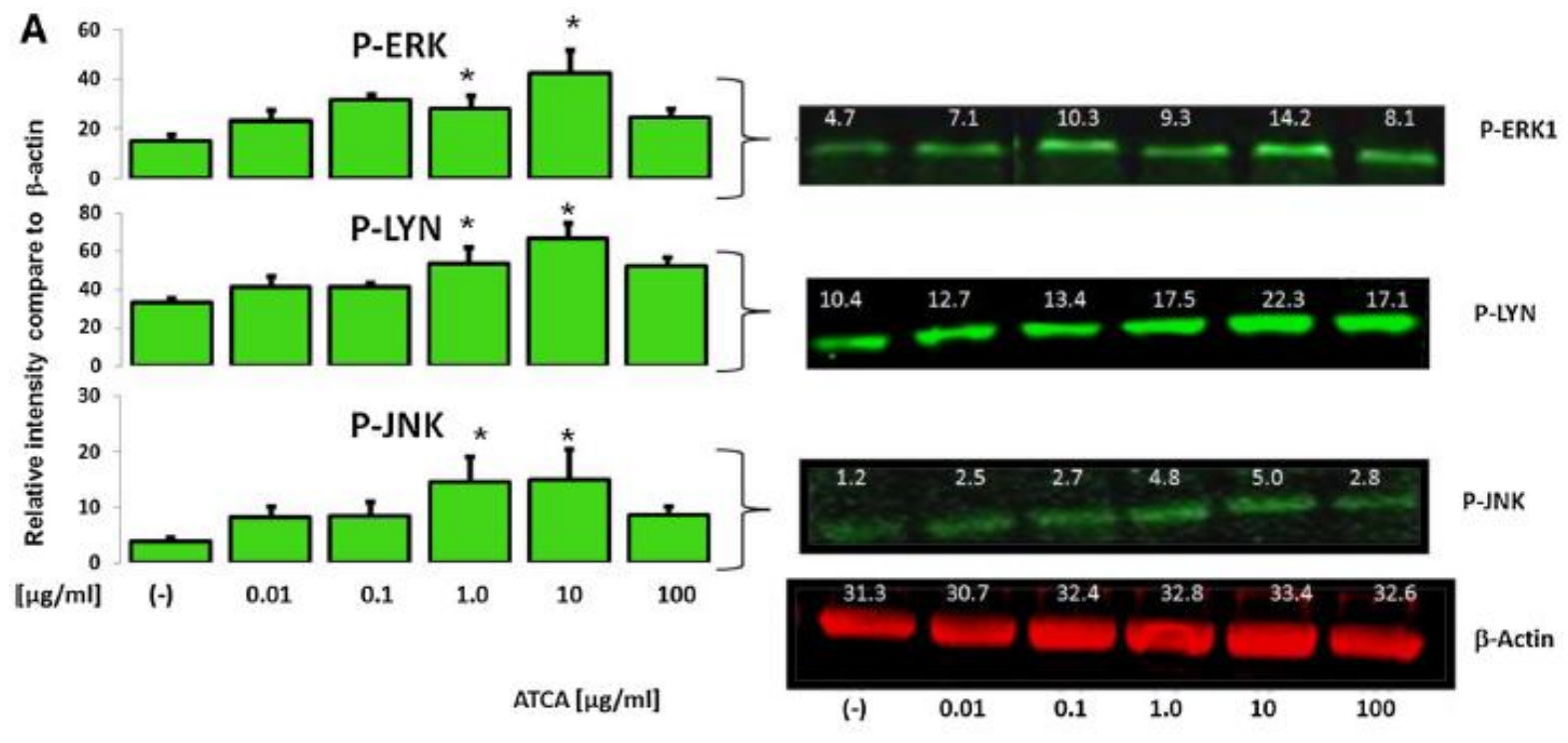

B

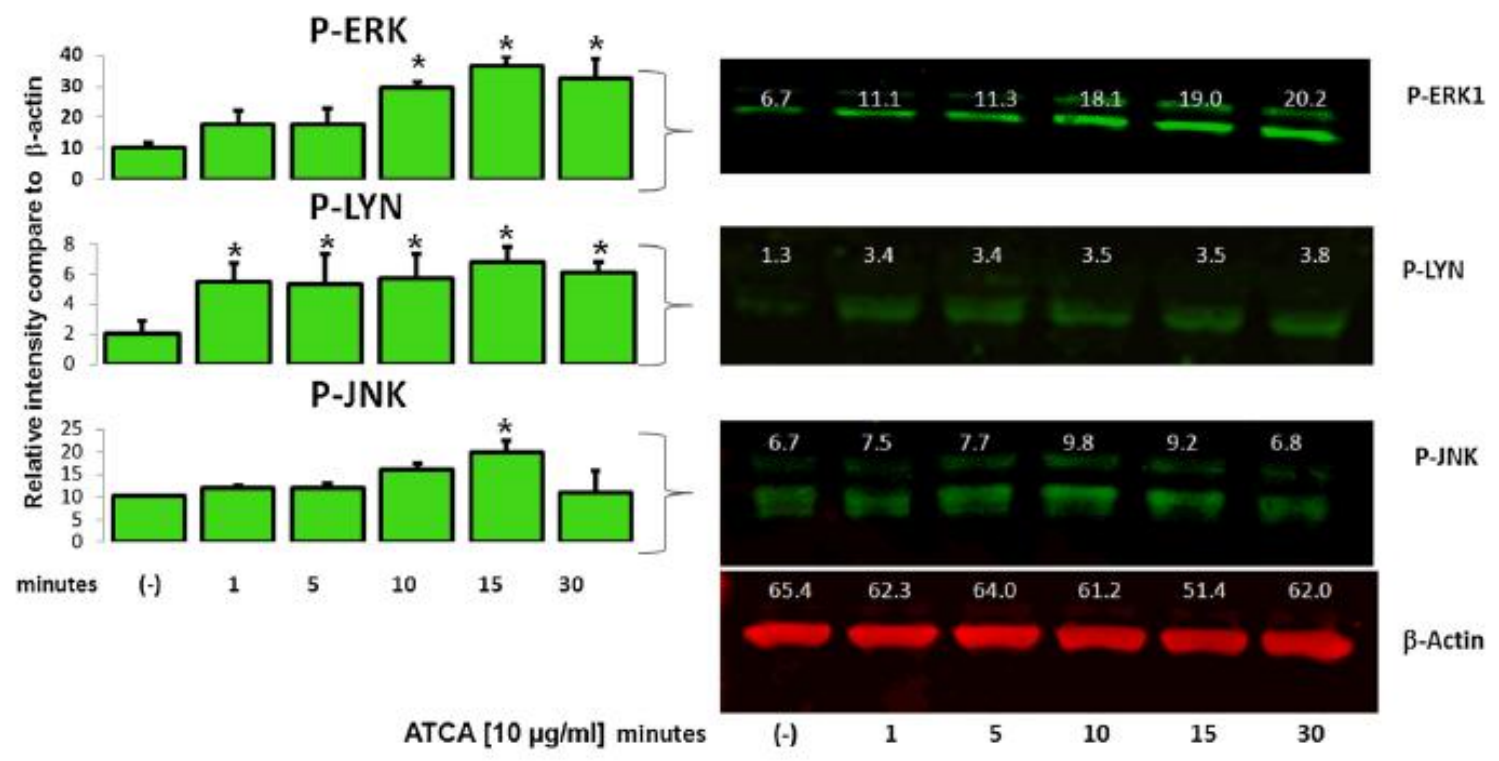

Figure 4.4. ATCA Activate CD36-Specific Signaling Intermediates. Foam cells were incubated with or without varying concentrations of ATCA (A; dose response) for 10 min or with $10 \mu \mathrm{g} / \mathrm{ml}$ ATCA (B; time course) for varying times. Cells were lysed and Western blotted using phospho-specific antibodies. Band intensities were quantified and the numbers represent the fold increase of each band compared to the housekeeping gene $\beta$-actin which did not demonstrate any change upon ATCA challenge. Results are representative of three separate experiments. 


\section{IV.3.2 ATCA detect atherosclerotic plaque in vivo}

We used ATCA to visualize atherosclerotic disease in ApoE -/- mice [80]. As seen in Figure 4.1A (top and middle), mice injected i.v. with the ATCA had a striking enhanced T1 image of the plaque attached in the mouse aorta that could not be seen prior to injection. Of note is the observation that the imaging agent accumulates over time, suggesting it circulates through the blood for periods long enough for biomarker targeting to occur (data not shown). Control compounds without the CD36 ligands demonstrated no accumulation in the vessel walls of ApoE -/- mice (Figure 4.1A-bottom). Quantification of the image intensity of ROI's appearing after ATCA injection (Figure 4.1B) demonstrates accumulation of the compounds occurs after $30 \mathrm{~min}$; one and two hour time-points demonstrated the optimal imaging time. When measuring the entire aorta the normalized mean intensity was very similar to what was observed in the bright spot ROI's (Figure 4.1C). This quantification clearly demonstrates the ATCA significantly increases the contrast representing plaque lesions.

Separate experiments were performed with non-diseased animals. In these experiments the ATCA and control were injected and the descending aorta imaged at the same time points. As seen in the bottom panels wildtype C57 mice did not demonstrate any signal enhancement in the descending aorta further demonstrating the specificity of the ATCA for plaque detection. These results suggest the ATCA specifically detect disease-induced plaque lesions that are not expressed in normal vessel walls. Histochemical evaluation of each animal revealed that plaque lesions were present in the 
diseased animals (Figure 4.1D; left) with the accumulation of CD36-and CD68-positive cells on the anterior portion of the plaque lesions (Figure 4.1D; middle/bottom).

IV.3.3 Tracking the in vivo fate and toxicity of plaque-targeting contrast agents

In order to determine the fate of the ATCA we performed whole body scans after i.v. injection as described above. In all experiments the injection was well tolerated in each group of mice with no noticeable adverse reactions observed. As determined using CMR, there was no accumulation of any of the compounds in any major organs (e.g. liver and kidneys) at the same time points measured in the ApoE -/- mice (Figure 4.5A). To confirm these results we performed ICP analysis on the organs of these animals after seven days post injection. As seen in Table $4.1 \mathrm{Gd}$ was only detected in the liver and kidneys demonstrating that approximately $85 \%$ of the ATCA is excreted from the body at this time-point.

Table 4.1. Total Percent Gadolinium in Tissue.

\begin{tabular}{lcc}
\multicolumn{3}{c}{ Day $\mathbf{7}(\mathbf{n}=\mathbf{4})$} \\
\hline Average & SD \\
\hline Heart & $9 \%$ & $\pm 6 \%$ \\
Spleen & $\mathrm{ND}$ & $\mathrm{ND}$ \\
Kidney & $\mathrm{ND}$ & $\mathrm{ND}$ \\
Lung & $3 \%$ & $\pm 2 \%$ \\
\hline Total Gd in tissue was valuate compared to total Gd injected ND-none & $\mathrm{ND}$ \\
detected. & $\mathrm{ND}$ &
\end{tabular}

In separate experiments in which high concentrations of ATCA were injected, there was no significant increase in serum activity of ALT and AST between the 
untreated and ATCA-treated animals indicating no liver toxicity (Figure 4.5B). Thus, the ATCA do not affect liver function and are mostly cleared from the body within five days after injection.

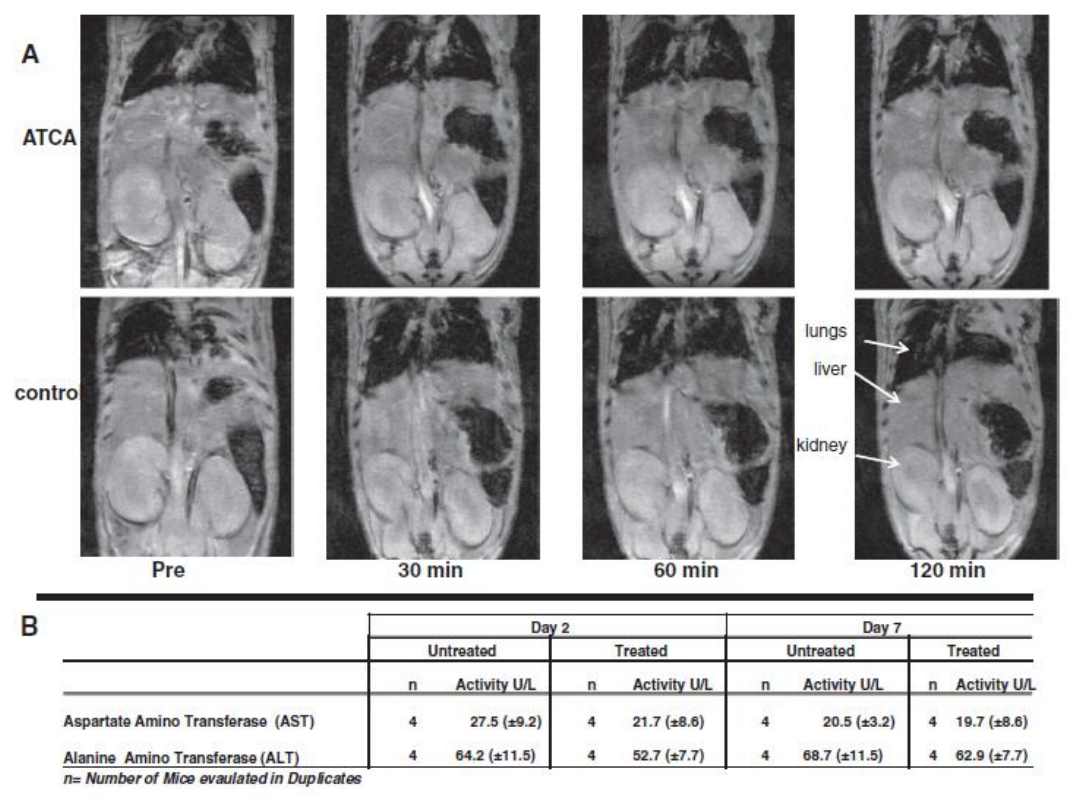

Figure 4.5. Plaque-Targeting Contrast Agents do not Accumulate in Major Organs. A. Representative plaque-targeting contrast agent and non-targeted control was injected as above and the mouse placed supine for whole body scan. Results are representative of four separate mice. B. C. ATCA does not accumulate in liver or induce toxicity. Mice were treated with or without $1000 \mu \mathrm{g} / 100 \mu \mathrm{l}$ of ATCA via tail vein injection. Two and six days later the serum was used for ALT and AST levels. Data is presented as an average of four (untreated) or four (treated) mice \pm SD. No increase in activity was observed between the untreated and treated samples. Activity is measure by Units/L obtained using linear regression from a standard curve.

\section{IV.4 Conclusions}

The results presented here demonstrate that atherosclerotic plaque can be detected in vivo using targeted, Gd-based molecular imaging nanomaterials and standard CMR 
techniques. Our rationale for developing ATCA was driven by the need for new ways to diagnose blood vessel plaque burden with existing technologies such as CMR. It is well established that atherosclerotic plaque express high levels of CD36 receptors for which ligands have already been identified. Importantly, these receptors normally and actively internalize the ligands (oxLDL); a prerequisite for accumulation of sufficient levels so that enhanced contrast can be observed. It is shown that this strategy was successful in identifying new ATCA which may provide new tools for physicians to detect plaque buildup in patients.

Magnetic resonance imaging is one of many techniques used to assess atherosclerotic plaque lesions in patients $[151,152]$. We functionalized the TMS through the addition of CD36 ligands which were hypothesized to home to macrophage foam cells found in atherosclerotic plaque $[75,76,153,154]$. Indeed, many studies have used this strategy of targeting diagnostics to macrophages through scavenger receptors as well as other macrophage-exp ressed biomarkers through several techniques including CMR [72, 153, 155-164]. For example, the Fayad group investigated the ability of macrophage scavenger receptor-targeted immunomicelles to assess macrophages in atherosclerotic plaque using CMR in vivo. The targeted micelles increased the signal intensity of atherosclerotic aortas $79 \%$ in ApoE- $-1-$ mice compared with only $34 \%$ using untargeted controls [153]. Others have targeted macrophages through the use of Lyp-1 peptides attached to a fluorescent dye [164]. Thus, targeting macrophages in atherosclerotic lesions is a valid strategy for developing diagnostics capable of detecting and quantifying plaque lesions. The advantages of using the CD36 receptor for targeting CMR contrast 
agents to plaque is its well established connection in atherosclerosis $[75,76]$, its high expression on plaque foam cells [76], and its ability to be internalized upon ligand binding [165] which allows the accumulation of the ATCA ligand even under physiological flow conditions as was shown in Figure 4.1. The key limitation presently is the lack of a specific biomarker (or biomarkers) for plaque lesions that will not crossreact with other sites in the body. An even more difficult challenge is identifying vulnerable plaque biomarkers.

We used the Gd-containing TMS as they provide a unique starting material for developing biomarker-targeting diagnostics [166]. The TMS offers several advantages over current Gd-based CMR contrast agents for use as targeted diagnostics. First, the toxic Gd (in cage) is separated from active targeting moieties (outside cage) by the carbon shell. Adding targeting ligands/moieties to current contrast agents may affect the ability of Gd to become free of the compound. Of course, further studies are needed for these molecular entities but we and others have not observed any toxicity of empty cage or TMS (Figure 4.5) using several in vitro and in vivo assays [32, 49, 167]. Second, the TMS are more sensitive than existing contrast agents with three $\mathrm{Gd} /$ molecule compared to one $\mathrm{Gd} /$ molecule in commercially available chelates [166]. Targeted imaging agents require strong signals by which to report the presence of an agent at a particular location. Third, the fullerene cage can be targeted to disease biomarkers or encapsulated into liposomes/micelles without compromising release of Gd into the body; a strategy already being used for targeting glioblastoma [168]. The chemistry for the addition of different moieties to the cage offers many possibilities for not only biomarker targeting of plaque 
accumulation but also detection of biomarkers associated with removal of lesions (e.g. peptidoglycans) so that new tools for evaluative diagnosis of this disease can be developed.

The specificity of the ATCA for targeting foam cells through CD36 is demonstrated three separate ways. First, ICP analysis of Gd within foam cells was confirmed in Figure 4.2B demonstrating that the CD36-ligand-decorated ATCA were significantly taken up within foam cells while the non-targeted ATCA were not. Second, we used FIB-SEM; a powerful technology that can be used to cut the cell open and detect nanomaterials, to further show the targeted ATCA is specifically internalized within foam cells were levels begin to diminish after $72 \mathrm{~h}$. This suggests the cells remain viable and can continue to exocytose as a normal macrophage would. Third, we show the targeted ATCA, and not the non-targeted, specifically activate signal transduction intermediates in the CD36 receptor pathway including the ligand binding-dependent phosphorylation of Erk, Lyn, and Jnk. Thus, the ATCA specifically detects and is taken up by macrophage foam cells though the CD36 receptor.

The use of ApoE -/- mice to study CMR contrast agents has been described extensively $[80,142]$. We demonstrate that the ATCA specifically recognize atherosclerotic plaque in diseased animals. No contrast was observed with non-targeted controls or in ATCA-treated, non-diseased animals. The signal enhancement occurs in a time-dependent fashion suggesting the foam cells are actively internalizing the ATCA via the CD36 scavenger receptor. While it has been reported that the lesion burden can vary among animals it was demonstrated that each animal tested had high levels of plaque 
lesions containing macrophages and CD36 receptors as identified using histochemistry and immunohistochemistry (Figure 4.1D). While each animal was verified to contain inflammation one limitation of this study was the small numbers of animals used. We are in the process of verifying these results in higher-order mammals (rabbits). The high power $7 \mathrm{~T}$ scanner used warrants further validation of the ATCA in a more clinically relevant scanner. However, the TMS have been studied in lower field magnets and demonstrate similar relaxivity and sensitivity $[169,170]$.

Investigation of biocompatibility and biodistribution of the TMS-based protein cages in vivo is critical for future clinical translation. To this end, the studies examining the toxicity of fullerenes on human systems are still emerging and the subject of debate $[167,171]$. Several in vivo publications suggest that (in general) fullerenes show no overt signs of toxicity up to $2000 \mathrm{mg} / \mathrm{kg}[99,100]$ and have been shown to extend the lives (11\%) and improve cognitive function of mice chronically treated with these compounds [17]. More recently, rats given fullerenes in olive oil lived over $90 \%$ longer than control mice [18]. We demonstrated that a single dose of ATCA, given at a 100 fold concentration higher than that needed for plaque imaging in vivo, caused no overt signs of toxicity, does not accumulate in the liver, and does not induce liver damage. These results are consistent with the observation that TMS injected i.v. into mice is passed through the kidney and into the urine (data not shown). The ATCA is mostly cleared from the body after seven days as demonstrated above. Similarly, the TMS were recently shown to have a favorable toxicity profile in vitro and in vivo [49]. Since it is anticipated that a patient will only be given a single injection before CMR is performed, these results 
are encouraging for future studies in humans. However, more studies are clearly needed to examine the long-term effects of fullerenes on biological systems. Efforts are focused on systematically identifying any acute and long-term toxicity issues with ATCA.

This study describes the synthesis and testing of a new class of ATCA based on a novel metallofullerene nanomaterial. In vitro screening was used to select high relaxivity compounds that were taken up by foam cells via the scavenger receptor CD36. The CD36 compounds were shown to target inflammatory lesions in ApoE -/- mice while the same compounds without the CD36 ligands did not. These and other similar studies discussed above suggest the rationale for targeting macrophage foam cell may provide physicians with new tools for non-invasively diagnosing and quantifying otherwise undetectable plaque lesions. These results also suggest the TMS may serve as a platform to attach other biomarkers to target and image disease-specific biomarkers. 


\section{CHAPTER V}

\section{A STEROID-MIMICKING FULLERENE THAT MEDIATES INHIBITION OF HUMAN LUNG MAST CELL RESPONES}

\section{V.1 Introduction}

The use of corticosteroids (also known as glucocorticosteroids, glucocorticoids, or steroids) is the hallmark treatment for patients suffering from many chronic inflammatory and immune diseases (such as asthma) yet the treatment response is extremely variable among patients. Specific subsets of patients are nonresponsive to such treatments and are termed steroid resistant. These patients often fail to respond to even extremely high dosages of inhaled corticosteroids (ICS), therefore much research has been focused on understanding the mechanisms of ICS action to better understand non-responsive patients [172]. Several different studies have found that the dual-specificity phosphatase 1 (DUSP1) is a prospective regulator of this ICS response [173-177]. Dual-specificity

phosphatase 1 is one in a family of protein phosphatases which has been shown in vitro to inactivate mitogen-activated protein kinases (MAPK) [174]. Upregulation of DUSP1

directly results in the de-phosphorylation/inactivation of mitogen-activated protein kinase (MAPK) via dephosphorylation of the threonine and/or tyrosine residues and thus acts as a regulator through a negative feedback mechanism [178-180]. The induction of the USP1 gene and subsequent inhibition of MAPKs result in the reduced expression of proinflammatory cytokines [181-183]. 
While ICS therapy remains a successful first line treatment for persistent cases of asthma, consistent use of steroids can have many harmful side effects. In addition, polymorphisms in DUSP1 expression are associated with clinical effectiveness of ICS therapy for asthma [175]. For patients nonresponsive to ICS therapy $\beta 2$-adrenergic receptor agonists are used. However, the link between DUSP1 expression and patient responses to ICS/ $\beta 2$-adrenergic receptor agonists is not completely understood. Many cell types respond to steroids by upregulating DUSP1, which dephosphorylates and inactivates both MAPK and JNK resulting in significant reductions in mediator release and the production of pro-inflammatory cytokines.

Lung mast cells (MC) are effector cells in the asthmatic response [23] and release of their asthma-triggering mediators has been shown to be inhibited by steroids [184, $185]$ and $\beta 2$-adrenergic receptor agonists [186]. The role of DUSP1 expression in activated human lung MC following pharmacological interventions has not been studied. Clues for a role for DUSP1 in MC-driven responses came from studies where MC from knockout mice lacking DUSP1 show enhanced degranulation and are highly susceptible to anaphylaxis [187]. Mast cell stabilizing nanomaterials were also shown to increase DUSP1 gene expression which paralleled inhibition of mediator release [22]. Based upon these similarities, we investigated the mechanism underlying $\mathrm{C}_{70}$-Tetrainositol $\left(\mathrm{C}_{70}-\mathrm{I}\right)$ inhibition of lung MC mediator release and compare its effects to those of steroids and $\beta 2$-adrenergic receptor agonists. As shown below C70-I inhibited both Fc\&RI-mediated degranulation and GM-CSF cytokine production which was paralleled with increases in DUSP1 expression. Furthermore, $\mathrm{C}_{70}$-I synergized with long-acting $\beta 2$-adrenergic 
receptor agonists (LABA) to potentiate this inhibition. These findings provide mechanistic insight into how $\mathrm{C}_{70}$-I can mediate $\mathrm{MC}$ degranulation and cytokine production and how DUSP1 polymorphisms could influence varying responses in patient ICS treatment [175] through the upregulation of lung MC DUSP1 levels.

\section{V.2 Methods}

\section{V.2.1 Fullerenes synthesis and characterization}

The $\mathrm{C}_{70}$-I was synthesized by conjugating four myo-inositol molecules (cis1,2,3,5-trans-4,6-cyclohexanehexol) to each $\mathrm{C}_{70}$-carbon cage via Bingel-Hirsch cyclopropanation reaction $[48,188,189] . \mathrm{C}_{70}$-I has two inositol moieties at each of the two poles of the oval-shaped $\mathrm{C}_{70}$ molecule. These myo-inositol moieties solubilize the $\mathrm{C}_{70}$ molecule in aqueous media. Myo-inositol has been shown to significantly reduce inflammation in two widely used animal models for inflammation [190]. The final compound was characterized using matrix assisted laser desorption ionization mass spectrometry (MALDI-MS), nuclear magnetic resonance (1H and 13C NMR), highperformance liquid chromatography (HPLC), Fourier transform infrared spectroscopy

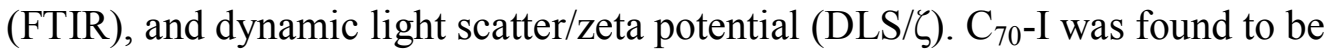
approximately $30 \mathrm{~nm}$ in diameter and fully dispersed in buffered saline, it has a molecular formula of $\mathrm{C} 100 \mathrm{H} 44 \mathrm{O} 32$ with $\mathrm{MW}$ of $1756 \mathrm{Da}$, and a zeta potential of -16.90 $\mathrm{mV}$ (Appendix A). Additionally, cell toxicity was assessed by incubation with concentrations shown effective for inhibition $\left(10^{-8}, 10^{-7}, 10^{-6}\right.$, and $\left.10^{-5} \mathrm{M}\right)$ and viability counts taken on days three, six, and nine. No toxicity was observed using trypan blue 
exclusions compared to control cells (Appendix A). Pre-incubation of MC with a fluorophore tagged- $\mathrm{C}_{70}-\mathrm{I}$ (Texas Red) revealed uptake of fullerenes by $\mathrm{MC}$ with high efficiency at concentrations down to $10^{-10} \mathrm{M}$ after $5 \mathrm{~h}$ of incubation (Appendix A).

\section{V.2.2 Gene microarray}

Mast cells were harvested from tissue received by the Cooperative Human Tissue Network and cultured using methods previously described [24-26]. Mast cells (N95\%; 1 $\times 10^{7}$ cell/condition; each condition performed in triplicate) were incubated with or without $\mathrm{C}_{70}-\mathrm{I}\left(10^{-5} \mathrm{M}\right.$ for $\left.16 \mathrm{~h} ; 37^{\circ} \mathrm{C} ; 6 \% \mathrm{CO}_{2}\right)$, washed in fresh warm $\left(37^{\circ} \mathrm{C}\right)$ media and supernatants removed (to remove pre-formed mediators), and fresh warm medium alone (negative) or medium containing anti-FceRI Abs $(1 \mu \mathrm{g} / \mathrm{ml})$ added for two hours at $37^{\circ} \mathrm{C}$. Cells were centrifuged, the supernatant and the pellet immediately frozen and microarray performed using the Human Whole Genome OneArray ${ }^{\mathrm{TM}}$ gene expression profiling service (Phalanx Biotech Group) report. Microarray analysis was performed and analyzed as described previously [22]. The UNCG Human Studies International Review Board approved all studies.

\section{V.2.3 Immuno-blotting and immuno-precipitation}

Mast cells $\left(\sim 3.0 \times 10^{6}\right.$ cells $\left./ \mathrm{ml}\right)$ were pretreated with or without $\mathrm{C}_{70}-\mathrm{I}$ and either cross-linked with anti-Fc\&RI Abs $(0.5 \mu \mathrm{g} / \mathrm{ml})$ or left untreated in a $37^{\circ} \mathrm{C}$ incubator with $6 \% \mathrm{CO}_{2}$. Cells were lysed using methods previously described [48, 105, 106, 191]. For immuno-precipitations (IP), lysates were pre-cleared with Protein A/G agarose beads, followed by overnight incubation with anti-DUSP1 (Abcam, Cambridge, MA) 
conjugated beads at $4^{\circ} \mathrm{C}$ and washed extensively to isolate DUSP1 proteins. Precipitates were subjected to SDS-PAGE (10\% SDS gels; Life Technologies, Grand Island, NY) and immuno-blotting (IB) as described [106]. For highly efficient and quantitative exposure, IR700 or IR800-conjugated secondary antibodies were measured using Licor's Odyssey infrared imaging system (Licor Inc. Lincoln, NE). The band intensities were quantified as described [192] and normalized to the house-keeping gene, $\beta$-actin.

\section{V.2.4 Mast cell mediator release}

Lung MC of the MCT (tryptase-positive, chymase-negative; predominant type seen in the alveoli of the lung N93\%) [193] were incubated for $16 \mathrm{~h}$ with or without $\mathrm{C}_{70}-\mathrm{I}$ and ICS alone (at increasing dosages to evaluate dose response), or in combination with $\beta 2$-adrenergic receptor agonists or both overnight $\left(16 \mathrm{~h} ; 37^{\circ} \mathrm{C} ; 6 \% \mathrm{CO}_{2}\right)$. Cells were washed and activated with optimal concentrations $(0.5 \mu \mathrm{g} / \mathrm{ml})$ of anti-FceRI Abs (3B4) for $2 \mathrm{~h}(\beta$-Hex $)$ or $16 \mathrm{~h}$ (cytokines) at $37^{\circ} \mathrm{C}$. Mediator release was measured as described previously $[105,194]$. Incubation times and concentrations were previously optimized and it was determined that 70-carbon fullerenes were taken up by $\mathrm{MC}$ through an endocytosis-dependent mechanism and persisted in the MC for up to one week, predominantly homing to the ER, but also to a lesser degree found in the mitochondria and lysosomes [21]. 


\section{V.3 Results}

\section{V.3.1 Pretreatment of mast cells with fullerenes upregulates DUSP1 gene and protein}

levels

Microarray analysis was used to obtain a broad overview of those FceRIassociated signaling molecules influenced by $\mathrm{C}_{70}$-I pre-incubation following FceRI activation [22]. Interestingly, $\mathrm{C}_{70}$-I caused a dramatic increase in expression of wide range of genes, including several within the DUSP family. Notably, DUSP1 increased relative gene expression levels approximately 55\% compared to cells that were not crosslinked with FceRI. Cells pre-incubated with $\mathrm{C}_{70}-\mathrm{I}\left(10^{-5} \mathrm{M}\right)$ for $16 \mathrm{~h}$ prior to activation increased gene expression to approximately $155 \%$ (Figure $5.1 \mathrm{~A}$ ). Next, a dose response was performed to find the optimal concentration of $\mathrm{C}_{70}$-I that could affect the protein expression of DUSP1 in MC. Cells incubated with $\mathrm{C}_{70}-\mathrm{I}$ at $10^{-5} \mathrm{M}$ were found to maximally increase DUSP1 protein expression (Figure 5.1B). These data were further supported by IB of the DUSP1 protein at 2, 4, and $6 \mathrm{~h}$, where the pre-incubation of MC with $\mathrm{C}_{70}$-I markedly increased protein expression by nearly $99 \%$ at 4 and $6 \mathrm{~h}$ (Figure 5.1C). Therefore, the $\mathrm{MC}$ inhibition observed with $\mathrm{C}_{70}$-I was paralleled by significant increases in DUSP1 expression. 

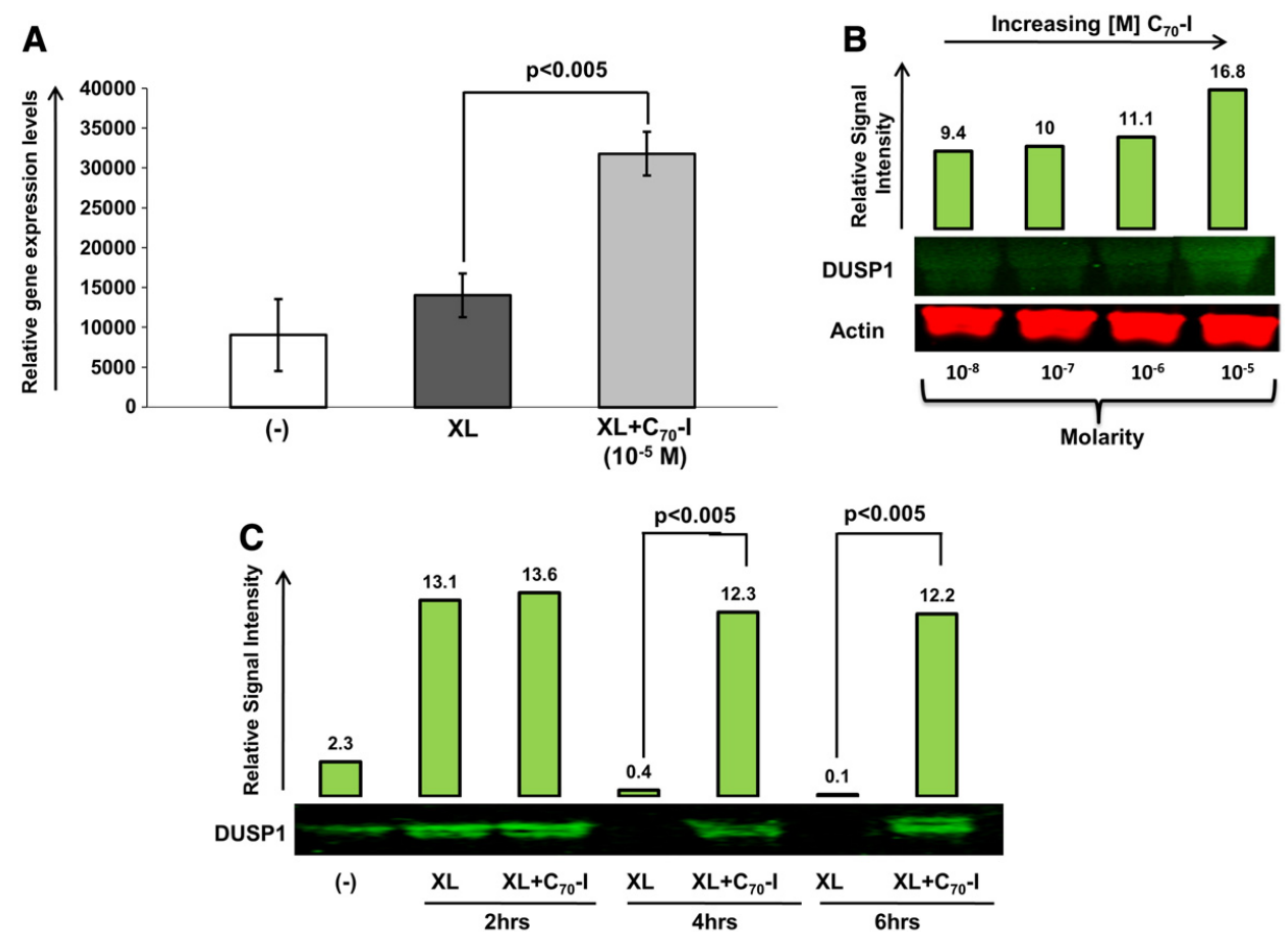

Figure 5.1. Mast Cell Pretreatment with $C_{70}$-I Increases DUSP1 Gene and DUSP1

Protein Expression. Fullerene $\left(\mathrm{C}_{70}-\mathrm{I}\left[10^{-5} \mathrm{M}\right)\right.$ treated and untreated MC were challenged with (XL) or without (-) anti-FceRI $(1 \mu \mathrm{g} / \mathrm{ml})$ for $2 \mathrm{~h}$. Cell pellets from each condition in triplicates were used for RNA isolation and gene microarray as

described. Gene microarray data conduction by Phalynx Biotechnology (A) demonstrates the increases in mean relative intensities of DUSP1 gene expression increases in $\mathrm{C}_{70}$-I pretreated samples (P b 0.005 ; \pm SD of six observed values). To evaluate dose responses of $\mathrm{C}_{70}$-I $(\mathrm{B})$, MC were pretreated with $\mathrm{C}_{70}-\mathrm{I}\left(\mathbf{1 0}^{-8}-10^{-5} \mathrm{M}\right)$ and lysed as described. The lysates were immuno-blotted with polyclonal rabbitanti-DUSP1 antibodies $(1 \mu \mathrm{g} / \mathrm{ml})$ and probed on the Odyssey infrared imaging system with anti-rabbit IR800 secondary antibodies (results typical in three separate immuno-blots). Relative pixel intensity (green bar graph) of the IR800 protein band expression of DUSP1 was quantified and normalized (Licor Odyssey imaging software) to the house-keeping gene, $\beta$-Actin to ensure equal protein levels were loaded into each lane (red band). To evaluate time course effects of $\mathrm{C}_{70}-\mathrm{I}$ (C), MC were cross-linked with (XL) or without (-) anti-FceRI $(1 \mu \mathrm{g} / \mathrm{ml})$ for 2,4 , or $6 \mathrm{~h}$ and immuno-blotted as described in (B). 
V.3.2 Fullerene upregulation of DUSP1 protein expression modulates activation of MAPK

To further understand the correlation between DUSP1 expression and MC inhibition with $\mathrm{C}_{70}$-I, experiments were performed examining DUSP1 downstream targets. Given that increases in DUSP1 activity will directly result in reduction of MAPK via de-phosphorylation of threonine and/or tyrosine residues, protein analysis of phosphorylated MAPK (p-MAPK) levels in $\mathrm{C}_{70}-\mathrm{I}$ MC pretreated with $10^{-5} \mathrm{M}$ (the concentration that maximally activated DUSP1 protein expression; Figure 5.1) was performed. As expected, phosphorylation of MAPK was dramatically reduced at the four and six hour time points (Figure 5.2A); the same time points that showed significant increase in DUSP1 expression (Figure 5.1C). At $6 \mathrm{~h}$ phosphorylation of MAPK was nearly $80 \%$ lower in cells treated with $\mathrm{C}_{70}-\mathrm{I}$ as opposed to those that remained untreated (Figure 5.2B) indicating the $\mathrm{C}_{70}$-I-induced upregulation of DUSP1 directly results in dephosphorylation of MAPK in MC. These results suggest that increases in DUSP1 from $\mathrm{C}_{70}-\mathrm{I}$ directly prevent phosphorylation of MAPK which is crucial for MC activation leading to mediator release. 

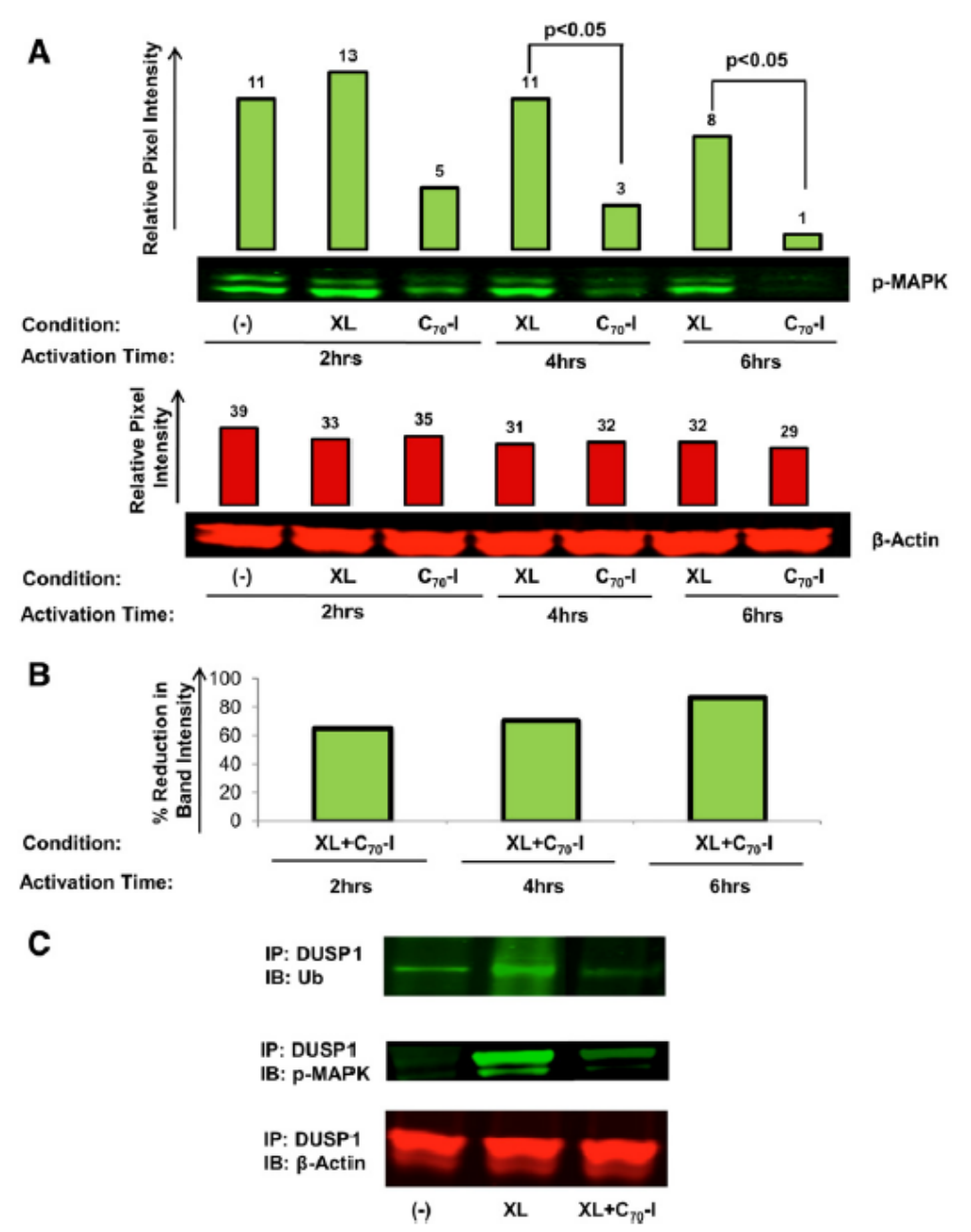

Figure 5.2. Mast Cell Pretreatment with $C_{70} . I$ Decreases Phosphorylation of MAPK1. Mast cells were pretreated with or without $\mathrm{C}_{70}-\mathrm{I}\left(10^{-5} \mathrm{M}\right)$, washed, and challenged with (XL) or without (-) anti-Fe\&RI $(1 \mu \mathrm{g} / \mathrm{ml})$ for 2,4 , or $6 \mathrm{~h}$. Lysates were immuno-blotted with polyclonal rabbit-antiphospho-MAPK1 antibodies (1 $\mu \mathrm{g} / \mathrm{ml}$ ) and probed on the Odyssey infrared imaging system with anti-rabbit IR800 secondary antibodies (A). Relative pixel intensity of the IR800 phospho-MAPK1 protein band (green band and bar graph) and IR700 house-keeping gene, $\beta$-Actin (red band and bar graph) was quantified. Percent reduction of MAPK1 in untreated $\mathrm{MC}$ and $\mathrm{C}_{70}$-I pretreated $\mathrm{MC}$ was normalized to the house-keeping gene and pixel density of was calculated (B). Results are typical of three separate immuno-blots. In

(C) cells were immuno-precipitated with anti-DUSP1 antibodies, run on SDSPAGE, and blotted with the indicated antibodies. 
To delineate the possible mechanism of action that $\mathrm{C}_{70}$-I inhibits phosphorylation of MAPK and subsequent cell activation, activated cells were subjected to immunoprecipitation (IP) with anti-DUSP1 antibody, followed by immuno-blotting (IB) with anti-ubiquiton (Ub), anti-phospo-MAPK, or anti- $\beta$-Actin. In FceRI-crosslinked cells DUSP1 protein was highly ubiquitinated (Figure 5.2C; compare lane one vs. lane two) which provides for a negative feedback mechanism for inhibition in other cell types [195]. However, when pre-incubated with $\mathrm{C}_{70}$-I there was a dramatic reduction in DUSP1 ubiquitination (Figure 5.2C; lane 3). These data indicate that the increased DUSP1 levels (and subsequent decrease in MAPK levels) in cells pre-incubated with $\mathrm{C}_{70}-\mathrm{I}$ are due to the inhibition of DUSP1 ubiquitination and subsequent degradation through the ubiquitinproteasome pathway.

\section{V.3.3 Fullerenes and corticosteroids inhibit FCeRI-mediated-MC degranulation}

Inhaled corticosteroids can also inhibit MC mediator release, but certain asthmatic patients are less responsive to ICS, due potentially to DUSP1 polymorphisms. Thus, based on the results suggesting a DUSP1-dependent inhibition of lung MC using $\mathrm{C}_{70}$-I, several commonly used ICS were compared in their ability to inhibit FceRI-mediator release. A battery of ICS was initially evaluated (data not shown) and revealed that fluticasone and budesonide had the most significant impact on blunting mediator release from $\mathrm{MC}$, therefore comparative studies were conducted on these two specific ICS. $\mathrm{C}_{70}-\mathrm{I}$ $\left(10^{-7}-10^{-5} \mathrm{M}\right)$ and fluticasone or budesonide $\operatorname{ICS}\left(10^{-6}\right.$ and $\left.10^{-5} \mathrm{M}\right)$ were capable of significantly reducing MC degranulation without affecting cell viability (N95\% using 
trypan blue exclusion) compared to their untreated counterparts (Table 5.1). Optimal inhibition was observed with $10^{-5} \mathrm{M}$ pretreatments of fluticasone, budesonide, and $\mathrm{C}_{70}-\mathrm{I}$ resulting in inhibitions of 55\%, 48\%, and 68\% respectively. Higher doses did not increase inhibition and resulted in some loss in cellular viability. These data demonstrate that $\mathrm{C}_{70}-\mathrm{I}$ is capable and comparable to ICS mono-therapy in preventing human lung MC FceRImediated degranulation.

\section{Table 5.1. Mean \% FceRI-Mediated Degranulation in $\mathrm{C}_{70}$-I or ICS (Fluticasone or Budesonide) Treated and Untreated Lung MC.}

\begin{tabular}{lll} 
Treatment/[Conc; M] & $\begin{array}{l}\text { Release } \\
\beta \text {-Hex (\%) } \pm \text { S.E. }\end{array}$ & $\begin{array}{l}\text { (\%) Inhibition } \\
\beta-H e x \pm \text { S.E. }\end{array}$ \\
\hline Spontaneous & $4.7 \pm 0.4$ & \\
Positive Control & $56.9 \pm 4.7$ & \\
Fluticasone [8] & $52.7 \pm 3.7$ & $7 \% \pm 6.8 \%$ \\
Fluticasone [7] & $42.3 \pm 2.2$ & $26 \% \pm 5.1 \%$ \\
Fluticasone [6]* & $34.9 \pm 2.3$ & $39 \% \pm 4.0 \%$ \\
Fluticasone [5]* & $25.6 \pm 0.5$ & $55 \% \pm 1.2 \%$ \\
Budesonide [8] & $56.6 \pm 3.8$ & $1 \% \pm 8.0 \%$ \\
Budesonide [7] & $41.8 \pm 3.5$ & $27 \% \pm 7.6 \%$ \\
Budesonide [6]* & $35.6 \pm 1.9$ & $37 \% \pm 3.4 \%$ \\
Budesonide [5]* & $29.6 \pm 2.9$ & $48 \% \pm 4.2 \%$ \\
$\mathrm{C}_{70}-\mathrm{I}[8]$ & $53.7 \pm 2.1$ & $6 \% \pm 3.9 \%$ \\
$\mathrm{C}_{70}-\mathrm{I}[7] *$ & $34.4 \pm 1.4$ & $40 \% \pm 4.1 \%$ \\
$\mathrm{C}_{70}-\mathrm{I}[6] *$ & $24.5 \pm 3.6$ & $57 \% \pm 7.2 \%$ \\
$\mathrm{C}_{70}-\mathrm{I}[5] * *$ & $18.2 \pm 1.5$ & $68 \% \pm 4.1 \%$ \\
\hline
\end{tabular}

Data shown are mean \pm standard error (S.E.) of $\beta$-Hex release and percent inhibition of $\beta$-Hex release compared to untreated, activated MC. Results are representative of three different experiments in triplicates.

ICS: Inhaled corticosteroid; MC: Mast Cell; M: Molar.

$* \mathrm{P}<0.05$.

$* * \mathrm{P}<0.005$. 
V.3.4 Co-incubations of fullerenes or corticosteroids with long-acting beta2-adrenergic receptor agonists inhibits FCeRI-mediated-MC degranulation

Combination techniques of ICS and LABA are commonly used by physicians to treat patients suffering from persistent asthma. Given the ability of $\mathrm{C}_{70}$-I to inhibit $\mathrm{MC}$ inflammatory mediator release through a DUSP1 mechanism and that patient responses to ICS are mediated by DUSP1 the next aim was to investigate the ability of $\mathrm{C}_{70}$-I to synergize with the LABA. Several LABA were investigated at different concentrations, the most efficient inhibitor LABA inhibitor, salmeterol, was selected for synergistic studies. As anticipated, MC treated with $\mathrm{C}_{70}$-I or ICS combined with LABA (dual-agent approach) revealed increased inhibition of degranulation compared to the single-agent approach. A side-by-side comparison of $\mathrm{C}_{70} \mathrm{I}$ and LABA or ICS and LABA combinatory incubation with MC is illustrated in Table 5.2. The dual treatment response resulted in optimal inhibition of degranulation at concentrations of $10^{-5} \mathrm{M}$ fluticasone, budesonide, or $\mathrm{C}_{70}$-I co-incubated with $10^{-5} \mathrm{M}$ salmeterol with statistically significant inhibitions of $59 \%, 57 \%$, and $75 \%$ respectively, on average an increased inhibition of cellular degranulation by $7 \%$ compared to the single-agent approach. Figure 5.3 represents a comparison of the single-agent (either ICS or $\mathrm{C}_{70}$-I alone) and dual-agent (ICS and LABA or $\mathrm{C}_{70}-\mathrm{I}$ and LABA) approach validating $\mathrm{C}_{70}-\mathrm{I}$ as a potential alternative to traditional ICS approaches for asthma therapy aimed at reducing lung MC hyperreactivity. In addition, these data illustrate both compounds' synergistic abilities with the LABA salmeterol to prevent FceRI-mediated lung MC degranulation. Although, statistical evaluation of the single-agent versus dual-agent approach did not approach 
significance, it was observed that inhibition was improved in most cases when MC were treated synergistically with LABAs and/or fullerenes/ICS. However, the evaluation fullerenes and LABA against ICS (fluticasone or budesonide) and LABA at a concentration of $10^{-7} \mathrm{M}$ produced statistically significant results (¥; in Figure 5.3), indicating that the fullerene molecule may be useful in blunting degranulation with similar efficiency to ICS.

\section{Table 5.2. Synergistic Effects on \% FceRI-Mediated Degranulation in Fullerene $\left(\mathrm{C}_{70}-\mathrm{I}\right)$ and LABA (Salmeterol) or ICS (Fluticasone or Budesonide) and LABA (Salmeterol) Treated and Untreated MC.}

\begin{tabular}{lll} 
Treatment/Conc; M] & $\begin{array}{l}\text { Release } \\
\beta \text {-Hex }(\%) \pm \text { S.E. }\end{array}$ & $\begin{array}{l}\text { (\%) Inhibition } \\
\beta \text {-Hex } \pm \text { S.E. }\end{array}$ \\
\hline Spontaneous & $6.5 \pm 0.5$ & \\
Positive Control & $35.9 \pm 3.1$ & \\
Fluticasone [8]/Salmeterol [5] & $35.7 \pm 0.6$ & $1 \% \pm 1.4 \%$ \\
Fluticasone| [7]/Salmeterol [5] & $26.8 \pm 1.5$ & $26 \% \pm 4.2 \%$ \\
Fluticasone [6]/Salmeterol [5]* & $18.1 \pm 1.2$ & $50 \% \pm 3.1 \%$ \\
Fluticasone [5]/Salmeterol [5]* & $14.8 \pm 2.9$ & $59 \% \pm 5.1 \%$ \\
Budesonide [8]/Salmeterol [5] & $37.8 \pm 1.8$ & No Effect \\
Budesonide [7]/Salmeterol [5] & $30.3 \pm 3.5$ & $16 \% \pm 5.5 \%$ \\
Budesonide [6]/Salmeterol [5]* & $19.4 \pm 1.5$ & $46 \% \pm 2.9 \%$ \\
Budesonide [5]/Salmeterol [5]* & $15.4 \pm 2.7$ & $57 \% \pm 4.2 \%$ \\
C $_{70}$-I [8]/Salmeterol [5] & $34.0 \pm 0.8$ & $6 \% \pm 1.2 \%$ \\
C $70_{70}$ [ [7]/Salmeterol [5]* & $15.9 \pm 2.0$ & $56 \% \pm 4.6 \%$ \\
C $_{70}$-I [6]/Salmeterol [5]** & $13.8 \pm 1.4$ & $62 \% \pm 2.7 \%$ \\
C $70_{70}$ - [5]/Salmeterol [5]** & $9.0 \pm 1.3$ & $75 \% \pm 4.0 \%$ \\
\hline
\end{tabular}

Data shown are mean \pm standard error (S.E.) of $\beta$-Hex release and percent inhibition of $\beta$-Hex release compared to untreated, activated MC. Results are representative of three different experiments in triplicates.

ICS: Inhaled corticosteroid; MC: Mast Cell; M: Molar.

$* \mathrm{P}<0.05$.

$* * \mathrm{P}<0.005$. 


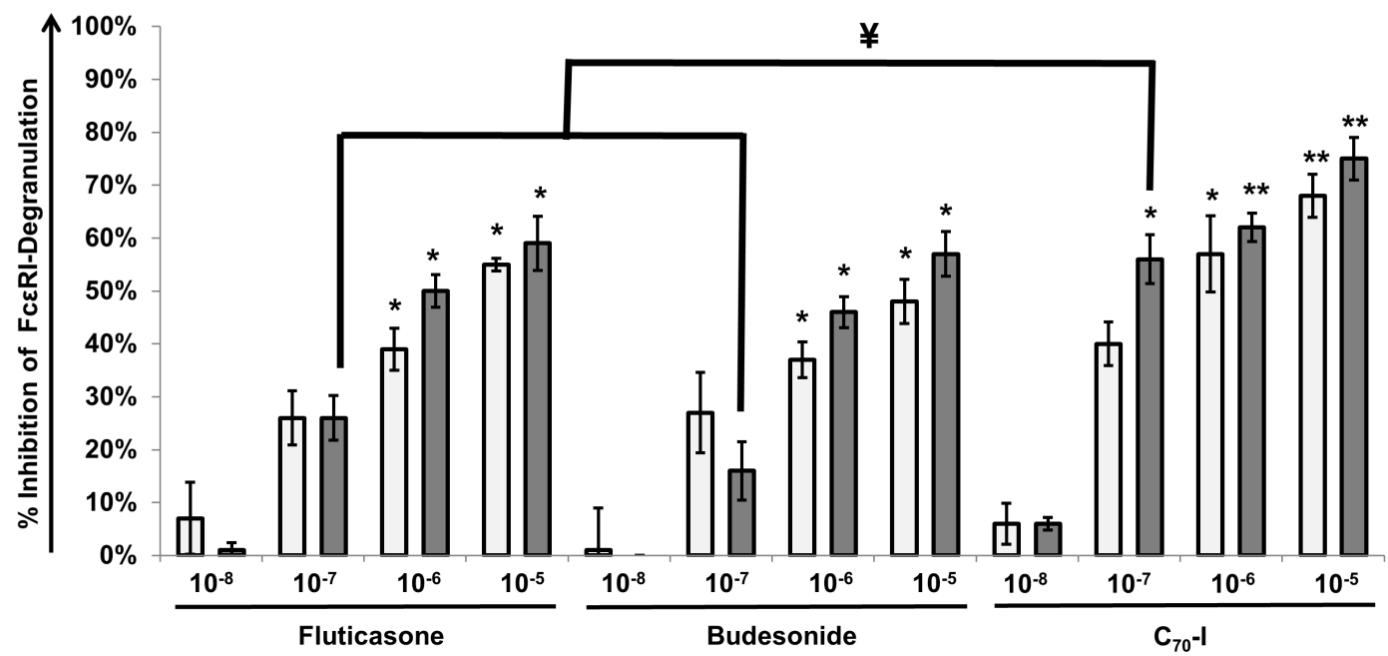

Figure 5.3. The Effects of MC Pretreatment with C70-I/ICS Individually or in Combination with LABA on Degranulation. MC were cultured with C70-I, fluticasone, or budesonide $\left(10^{-8}-10^{-5} \mathrm{M}\right)$ or C70-I, fluticasone, or budesonide at $\left(10^{-8}-10^{-5} \mathrm{M}\right.$;) in combination with salmeterol $\left(10^{-5} \mathrm{M}\right)$; each pretreatment condition as well as untreated MC controls (Spontaneous and Positive) was challenged with (XL) or without (-) anti-FceRI $(1 \mu \mathrm{g} / \mathrm{ml})$ for $30 \mathrm{~min}$ at $37^{\circ} \mathrm{C}$ and supernatants were collected to measure degranulation as described in Methods. All studies were performed using at least three separate MC donor cultures in triplicates. Statistical significance indicated with $*(\mathbf{P}<0.05)$ and $* *(\mathbf{P}<0.01)$ using the Student's test to compare respective treatment to untreated positive (FceRI) controls. Statistical significance indicated with $¥(P<0.05)$ using the Student’s $t$ test to compare efficacy ICS versus fullerene treatment at respective concentrations.

\section{V.3.5 Fullerenes and corticosteroids inhibit FCeRi-mediated-MC GM-CSF cytokine} production

Mast cells produce GM-CSF in response to inflammatory stimuli; it is observed that ICS therapy can suppress the release of inflammatory mediators. 1 Therefore, the ability of $\mathrm{C}_{70}$-I and ICS to reduce lung MC FceRI-mediated-GM-CSF release was investigated. $\mathrm{C}_{70}-\mathrm{I} / \mathrm{ICS}$ were evaluated separately and in 
conjunction with a LABA. As expected, ICS pre-incubation resulted in a dose dependent, statistically significant reduction in mediator release when compared to untreated controls (Figure 5.4A). Pre-incubation with $\mathrm{C}_{70}-\mathrm{I}$ at increasing doses resulted in greater inhibition of mediator release than observed in ICS treatments at similar concentrations (Figure 5.4A). The synergistic effect of $\mathrm{ICS} / \mathrm{C}_{70}-\mathrm{I}$ co-incubation with $\mathrm{LABA}$ resulted in greater inhibition of GM-CSF production (Figure 5.4B), just as observed in the degranulation assays. A comparison of the single-agent and dual-agent approach is depicted in Figure 5.4C demonstrating that $\mathrm{C}_{70}$-I has similar GM-CSF suppressive abilities as traditional ICS, as well as an ability to synergize with LABA to further inhibit cytokine production. Albeit, as was observed in the degranulation studies statistical significance was not revealed when comparing the single-agent and dual-agent therapies, it was found that lower concentrations of $\mathrm{C}_{70} \mathrm{I}\left(10^{-8}\right.$ and $\left.10^{-7} \mathrm{M}\right)$ with or without LABAs produced statistically significant inhibition when compared to the equivalent ICS (with or without LABA) treatment (¥, Figure 5.4C). 

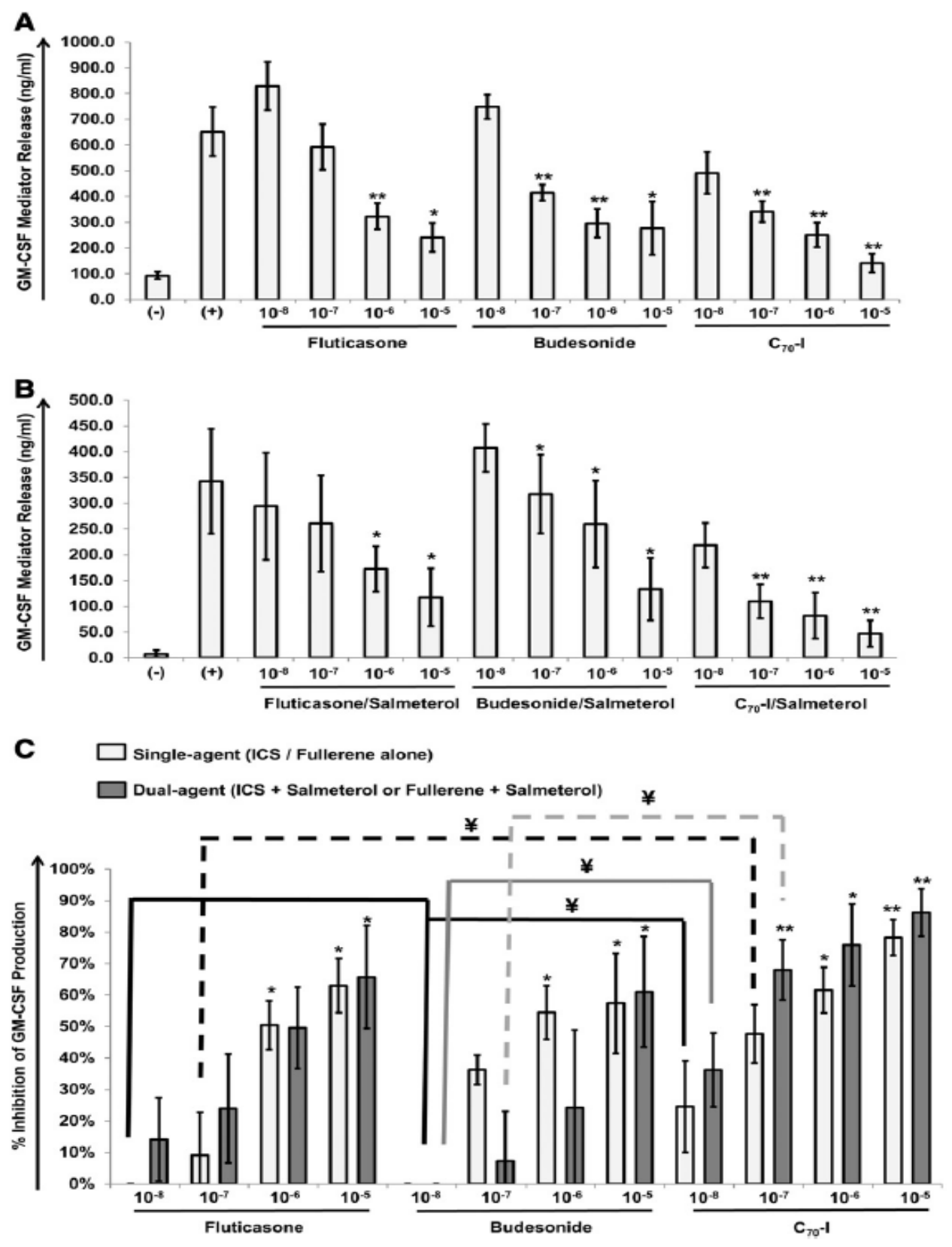

Figure 5.4 - The Effects of MC Pretreatment with C70-I/ICS Individually or in Combination with LABA on GM-CSF Cytokine Production. MC were cultured with $\mathrm{C}_{70}$-I, fluticasone, or budesonide at $\left(10^{-8}-10^{-5} \mathrm{M}\right)(\mathrm{A})$ or $\mathrm{C}_{70}$-I, fluticasone, or budesonide at $\left(10^{-8}-10^{-5} \mathrm{M}\right)$ in combination with salmeterol $\left(10^{-5} \mathrm{M}\right)(\mathrm{B})$; each pretreatment condition as well as untreated MC controls (Spontaneous and Positive) was challenged with (XL) or without (-) anti-FceRI $(1 \mu \mathrm{g} / \mathrm{ml})$ for $30 \mathrm{~min}$ at $37^{\circ} \mathrm{C}$ and supernatants were collected to measure mediator release as described. Values are presented as total mediator release $\mathrm{ng} / \mathrm{ml}$ and are representative of three 
experiments analyzed in triplicate. Percent inhibition of GM-CSF production of fullerene/ICS alone (white bar) and fullerene/ICS in combination with LABA is displayed in (C) Statistical significance indicated with $*(\mathbf{P}<0.05)$ and $* *(\mathbf{P}<0.01)$ using the Student's $t$ test to compare respective treatment to untreated positive

(FcERI) controls. Statistical significance indicated with $¥(P<0.05)$ using the Student's t test to compare efficacy of ICS versus fullerene treatment at respective concentrations.

\section{V.4 Conclusions}

Inhaled corticosteroids are regarded as the hallmark treatment for patients suffering from asthma. Their beneficial effect has been shown to be due in part to the inhibition of proinflammatory genes. Mast cells play a key role in IgE-dependent inflammatory diseases such as asthma. In many cases, MC mediate both the sustained and immediate inflammatory responses via degranulation and production of cytokines. Stimulation through the IgE-receptor, FceRI, results in the activation of MAPK and subsequently induces degranulation of preformed inflammatory mediators. The role that ICS play in modulating MC activation is of much interest, yet a defined understanding of the inhibitory mechanism of ICS at the cellular level has not been established. However, one protein, DUSP1, has been given significant attention $[172,177,181]$ Here it is shown that $\mathrm{C}_{70}-\mathrm{I}$ upregulates the DUSP1 gene in human lung $\mathrm{MC}$ resulting in higher protein levels and lower MAPK activation through an ubiquitination pathway. Furthermore, combinatory treatments of ICS and LABA can further inhibit MC activation and similar inhibition was observed when pre-treating with $\mathrm{C}_{70}$-I, instead of ICS.

Part of the impetus for these studies was the often unpredictable and varied side effects of ICS in asthma patients [196] as well as the donor variability in the benefit of 
ICS in some patients [175]. This has led to efforts to separate the desired antiinflammatory effects of ICS [197] and better understand the link between DUSP1 polymorphisms and their link between asthma lung function. Our studies have demonstrated that fullerenes are not toxic in vitro or in vivo $[32,49,198,199]$ but instead have an overall anti-inflammatory effect. Additionally, fullerene uptake is not receptor mediated, however it is endocytosed by the cell [21]. Given that this anti-inflammatory effect is now demonstrated in human lung $\mathrm{MC}$, both alone and in synergy with LABA, they may provide an alternative to ICS for patients that are steroid resistant without the unwanted side effects observed in high-dosage therapies. It is established the antiinflammatory actions of ICS are known to modulate gene expression via the glucocorticoid receptor (GR) [181]. Side effects of ICS are commonly attributed to gene induction by ligand-activated GRs [181]. While the ICS treatment strategy is deemed an extremely potent anti-inflammatory therapy, their use often comes with some reticence from physicians due to the range and severity of their side effects. Furthermore, it has been shown that mutated GRs have failed to activate the ICS response in a subset of patients [181]. Consequently, the non-receptor mediated mechanism of fullerenes may kinetically favor therapeutic intervention in cases of patients that fail to respond adequately or inversely to traditional ICS treatment regimes. Additionally, the differences in mechanisms between $\mathrm{C}_{70}-\mathrm{I}$ and ICS, although still in need of further investigation, may be advantageous for a $\mathrm{C}_{70}$-I-inspired therapy through a decrease in ICS-associated side effects. 
As a protein tyrosine phosphatase, DUSP1 is known to modulate immune responses. Specifically, DUSP1 is considered an ICS-induced phosphatase that is capable of inhibiting phosphorylation of MAPK in MC as first identified by Kassel et al. [200]. Furthermore, clinical effectiveness of ICS in patients with DUSP1 polymorphisms can be variable, suggesting that new therapies should be examined to help better control disease progression [175]. The $\mathrm{C}_{70}$-I was capable of increasing both gene expression and protein levels of the inhibitory regulator DUSP1, just as in ICS therapies. Pre-incubation of FceRI challenged $\mathrm{MC}$ with $\mathrm{C}_{70}$-I resulted in greater than a $50 \%$ upregulation of DUSP1 gene expression when compared to those untreated, which was analogous to high levels of DUSP1 protein expression in challenged MC at early (4 and $6 \mathrm{~h}$ ) time-points. As expected, the upregulation of DUSP1 caused significant (greater than 65\%) reductions in MAPK activation at these same time-points, suggesting that $\mathrm{C}_{70}$-I could function similarly to ICS intervention in modulating MC responses to stimuli.

The gene and protein expression results led to examination of commonly used in vitro functional assays to compare two ICS, fluticasone and budesonide, to $\mathrm{C}_{70}$-I as a possible alternative to traditional asthma-ICS regulated therapy. Being that both compounds were capable of mediating the phosphatase DUSP1, we sought to discover if $\mathrm{C}_{70}$-I could be a successful candidate for modulating MC-degranulation and cytokine production. Specifically, optimal doses of all three interventions, fluticasone, budesonide, and $\mathrm{C}_{70}$-I result in significant inhibitions of degranulation by $55 \%, 48 \%$, and $68 \%$ respectively, with fullerenes being nearly $20 \%$ more effective than traditional ICS treatments. These results were paralleled in GM-CSF cytokine production, where optimal 
concentrations of fluticasone and budesonide inhibit release by $63 \%$ and $57 \%$ respectively, the same concentration of $\mathrm{C}_{70}$-I revealed nearly $30 \%$ greater inhibitions with significant reductions in cytokine production by $78 \%$. In light of the degranulation and cytokine release assays, fullerenes may be an alternative to traditional ICS treatment. Overall, the fullerene intervention was capable of providing statistically significant reductions in MC-mediator release when compared to ICS treatment at the lower concentrations evaluated $\left(10^{-7} \mathrm{M}\right)$. In situations of persistent asthma, patients are often given a dual-therapy of ICS and LABA. Therefore, it was considered important to evaluate the ability of $\mathrm{C}_{70}$-I to synergize with LABA. Consequently, these strategies mirrored those commonly observed in combinatory therapies of ICS/LABA. Such that $\mathrm{C}_{70}$-I co-incubation with the LABA, salmeterol, resulted in increased inhibitions compared to fullerene mono-therapy. The synergistic effects were similar to those in the ICS/LABA dual-therapeutic strategy, fluticasone/salmeterol, or budesonide/salmeterol, while not statistically significant, repeatedly revealed enhanced inhibition of degranulation between $4 \%$ and $10 \%$ respectively when compared to the mono-therapeutic approaches. $\mathrm{C}_{70}$-I combinatory therapies with salmeterol resulted in nearly a $10 \%$ increase in inhibition of degranulation compared to $\mathrm{C}_{70}-\mathrm{I}$ alone. These same trends were observed in cytokine production. Taken together, each of these results provides a heretofore undiscovered mechanism for controlling asthma using novel water-soluble fullerenes that are functionally similar to the present first-line treatment method, ICS.

Further studies will be required to understand how $\mathrm{C}_{70}$-I functions to increase DUSP1 levels after pretreatment; however it is proposed that C70-I offers some 
protective qualities that prevent DUSP1 ubiquitination and subsequent activation-induced degradation. Following MC-activation, untreated cells displayed a rapid increase in DUSP1 production, a production that diminishes nearly $95 \%$ after two hours and $100 \%$ after four hours. However, the duration of this spike in production persists significantly longer in $\mathrm{C}_{70}$-I treated $\mathrm{MC}$ as displayed in Figure 6.1C. It is proposed that $\mathrm{C}_{70}-\mathrm{I}$ can prevent proteolysis degradation of the phosphatase, allowing it to act on MAPK well into six hours after stimulation with FceRI. Our efforts are now focused on understanding what $\mathrm{C}_{70}$-I interacts with directly or if it is indirectly reducing ubiquitination through another mechanism, possibly reduced oxidative stress as has been demonstrated in other cell types [201].

In conclusion, these studies reveal that strategically functionalized fullerene nanomaterials, like ICS, can control human lung MC mediator release through a DUSP1mediated mechanism. However, unlike ICS, their efficacy is not mediated through the glucocorticoid receptor. Since the fullerenes are taken up by $\mathrm{MC}$ via endocytosis, this may prove beneficial in patients with receptor mutations, who subsequently fail to respond to ICS treatment. These findings show that using water-soluble fullerenes with side-chain moieties such as $\mathrm{C}_{70}$-I could be pursued as a non-steroidal based therapy for patients that fail to respond clinically or respond adversely to ICS treatment. Additionally, these findings may not only provide a more robust controller therapy for patients with asthma, but a therapy with reduced adverse effects commonly associated with ICS treatment. 


\section{CHAPTER VI}

\section{INHIBITION OF INFLAMMATORY ARTHRITIS USING FULLERENE NANOMATERIALS}

\section{VI.1 Introduction}

Oxygen metabolism has an important role in the pathogenesis of inflammatory arthritis and therefore therapies that target its dysregulation have been investigated as potential treatments. Reactive oxygen species produced in the course of cellular oxidative phosphorylation, and by activated phagocytic cells during oxidative bursts, exceed the physiological buffering capacity and result in oxidative stress $[202,203]$. Various forms of antioxidant therapy have demonstrated promising results in experimental arthritis models [204-208]. The polyphenolic fraction of green tea containing potent antioxidants reduces symptoms of collagen-induced arthritis [209]. A traditional Mediterranean diet relatively high in antioxidants improved RA disease activity and functional status after three months compared with a standard 'Western' diet [210]. In a separate study of patients with RA, antioxidant supplementation with vitamin A, E, and C increased plasma antioxidant levels with a corresponding decrease in malondialdehyde, a marker of

oxidative stress; however, a clinical response was not reported [211]. Carvedilol, an adrenergic antagonist with antioxidant/anti-inflammatory properties effectively suppressed inflammation in two arthritis models [204]. More recently however, it was shown that water-soluble $\mathrm{C}_{60}$ fullerene prevents degeneration of articular cartilage in 
osteoarthritis (OA) in rabbits [212] but no studies have examined fullerenes in inflammatory arthritis.

The cellular interplay leading to inflammatory arthritis is complex. The synovium of many patients with rheumatoid arthritis (RA) exhibits an increase in the number of synovial mast cells (MC), in some cases representing 5\% or more of the expanded population of total synovial cells $[38,213]$. The number of accumulated MC differs substantially from patient to patient, in general varying directly with the intensity of joint inflammation [214]. Accompanying the increased numbers of MC, their mediators are also present at higher concentrations in the synovial fluid of inflamed human joints. These mediators include histamine, tryptase, and TNF- $\alpha$ all readily elicited from MC upon exposure to various immunological and non-immunological stimuli $[26,215,216]$. TNF- $\alpha$ activated synovial fibroblasts contribute to the initiation and perpetuation of inflammatory arthritis [217]. Several studies suggest that synovial fibroblasts demonstrate changes in morphology and function in RA patients through stimulation of both inflammation and tissue damage [218]. Lastly, osteoclasts are cells of the monocyte/macrophage lineage that are directly responsible for the bone destruction in inflammatory arthritis; therapies that reduce osteoclast function are being investigated as treatments for patients with inflammatory arthritis [219]. Reactive oxygen species act as intracellular signaling molecules in the regulation of RANKL-dependent osteoclast differentiation involving NF-kB [220-223]. In short, a clear connection exists between oxidative stress and the underlying mechanisms leading to inflammatory arthritis. 
Fullerenes or "Buckyballs" are one class of nanomaterials that represent the third allotrope (different structural modifications and arrangements of the same chemical elements) of carbon. Previous studies have demonstrated that fullerene derivatives can stabilize human $\mathrm{MC}$ depending on the structure of the chemical moieties added to the carbon cage $[20,22]$. Given that fullerene derivatives have general anti-inflammatory properties through reductions in ROS levels and the blunting of the NF-kB signaling pathway $[15,20,32,48]$ it was hypothesized fullerene derivatives could ameliorate inflammatory arthritis. To test this hypothesis, a panel of water soluble fullerene derivatives were developed and tested them in vitro for their ability to alter mediator release from arthritis-inducing cells including $\mathrm{MC}$ and synovial fibroblasts from RA patients, as well as their effect on human osteoclast formation. The best candidates were selected for their ability to attenuate inflammatory arthritis in vivo using the $\mathrm{K} / \mathrm{BxN}$ autoimmune model for arthritis and the CIA model for arthritis $[44,53]$. It is demonstrated that the ability of fullerene derivatives to inhibit inflammatory cell mediator release was dependent on the moieties added to the carbon cage. The ability to inhibit in vitro mediator release translated to in vivo efficacy only in the $\mathrm{K} / \mathrm{BxN}$ induced mice (regardless of the presence of MC). Collagen-induced arthritic mice showed no reductions in disease onset or progression when treated with fullerene derivatives.

The ability of the fullerene derivatives to localize to inflamed joints was demonstrated using dye-conjugated fullerene derivatives which did not accumulate in other organs. Thus, our results suggest rationally designed fullerene derivatives may provide an effective therapeutic option for the treatment of inflammatory arthritis by 
targeting ROS to prevent stimulation of pro-inflammatory cytokines, osteoclast formation, and stabilizing critical cells involved in RA progression.

\section{VI.2 Methods}

\section{VI.2.1 Fullerene derivatives}

A panel of fullerene derivatives was synthesized at Luna Innovations and characterized for particle size using dynamic light scattering (Malvern Instruments, Zetasizer Nano ZS, Westborough, Massachusetts, USA), qNano (Izon Science, qNano, Cambridge, Massachusetts, USA) and nano particle tracking analysis, (Malvern Instruments, Nanosight LM10, Westborough, Massachusetts, USA), zeta potential (Malvern Instruments, Zetasizer Nano ZSP, Westborough, Massachusetts, USA), NMR (Agilent Technologies, 400 Mhz NMR Spectrometer, Santa Clara, California, USA), and FT-IR (Agilent Technologies, Varian 670 FT-IR, Santa Clara, California, USA). A representative physiochemical characterization schematic for the two fullerene derivatives used for the in vivo studies (TGA and ALM) is shown in $[32,48]$.

\section{VI.2.2 Inflammatory mediator release from BMMC and human MC}

The activation of MC through Fce receptors is one constituent that drives inflammatory arthritis. Unlike primary human MC, mouse MC express FceRIII and may be stimulated to release mediators via this receptor in culture, although activation is constrained by the inhibitory receptor FceRII [193]. Thus, BMMC were generated from FceRII-deficient mice [44]. Cells were incubated with or without fullerene derivatives 
overnight $[10 \mu \mathrm{g} / \mathrm{ml}$; [22]], washed and rat anti- FcERII/III $(2.4 \mathrm{G} 2,1 \mu \mathrm{g} / \mathrm{ml})$ added for 2 h. Immune complex (IC) stimulation was mimicked by the addition of donkey anti-rat (DAR; $1 \mu \mathrm{g} / \mathrm{ml}$ ) added for $30 \mathrm{~min}$ (degranulation) or $24 \mathrm{~h}$ (cytokine) and cellular lysates prepared [224] collected and IL-1 $\beta$ measured using ELISA as described [22]. Human connective tissue MCTC [193] were generated from skin and stimulated with immune complexes (IC) and mediator release measured as described [194]. Initial experiments determined optimal concentrations of IC for human MC mediator release was $8.8 \mu \mathrm{g} / \mathrm{ml}$ antibody with $0.13 \mu \mathrm{g} / \mathrm{ml}$ NP-BSA (not shown).

\section{VI.2.3 Synovial fibroblast cytokine production}

Human fibroblasts (PC37303A1-S-Passage 1) from the synovium of RA patients $(n=5)$ having joint replacement surgery (Asterand, Detroit, Michigan, USA) were plated in triplicate on a 96 well plate using RPMI complete medium. Cells were incubated overnight with or without fullerene derivatives at various concentrations, washed, and activated with or without $10 \mathrm{ng} / \mathrm{ml}$ of TNF- $\alpha$ for $12 \mathrm{~h}$. The supernatants (in triplicate) were assayed for IL-6 and IL-8 using ELISA [105].

\section{VI.2.4 Osteoclast differentiation}

Human osteoclasts were obtained from peripheral blood mononuclear cells (PBMC) as described previously [225]. Mononuclear cells were cultured in complete DMEM containing RANKL (25 ng/ml) and M-CSF (25 ng/ml). Fullerene derivatives were added at varying concentrations and remained in the medium until the end of the experiment (eight days). Cells were washed, cytocentrifuge preparations made, and 
analyzed for tartrate resistant acid phosphatase (TRAP) activity by cytochemistry (Sigma Aldrich, Acid Phosphatase Leukocyte assay, St. Louis, Missouri, USA). At eight days multinucleated cells containing three or more nuclei were counted. TRAP-positive cells containing three or more nuclei were considered to be differentiated osteoclasts. Experiments were done in triplicate, 25 microscopy fields at $40 \times$ magnification were evaluated for each sample. Quantitation of osteoclasts TRAP staining was performed using the infrared imaging analysis (Li-Cor Biosciences, Odyssey® Imaging CLx System, Lincoln, Nebraska, USA).

\section{VI.2.5 Measuring NF-KB levels using in-cell Western}

Human MC were plated in triplicate in 96-well plates and incubated as above with fullerene derivatives, washed, and activated with or without FceR stimulation for $24 \mathrm{~h}$ as above. After treatment, cells were fixed by adding formalin to a final concentration of $3.7 \%$ formaldehyde and incubated at room temperature for 10 minutes. After fixation, the plates were washed with PBS then permeabilized (PBS containing 0.1\% Triton-X-100) by washing three times for 10 min on a bench top shaker and finally rinsed once with PBS containing $0.1 \%$ Tween 20 . Cells were blocked using $50 \mu \mathrm{L} /$ well blocking buffer (Li-Cor Biosciences, Odyssey® Blocking Buffer, Lincoln, Nebraska, USA) for one hour at room temperature. Primary antibodies, $\beta$-Actin (1:1000) (Cell Signaling Technology, Danvers, Massachusetts, USA) and NF-kB (1:500) (Cell Signaling Technology, Danvers, Massachusetts, USA), incubated overnight at $4^{\circ} \mathrm{C}$ using $20 \mu \mathrm{L} /$ well, washed three times with $100 \mu \mathrm{L} /$ well PBS-Tween, and centrifuged at room temperature. Secondary 
antibodies were prepared as for Western blotting with a few modifications: IRDye $680 \mathrm{CW}$ and $800 \mathrm{CW}$ conjugates of goat-anti-mouse-IgG (Li-Cor Biosciences, Lincoln, Nebraska, USA) were used at 11000 dilution for detection of antibody targets in the 800 and $700 \mathrm{~nm}$ channels (green and red respectively). Plates were incubated with $20 \mu \mathrm{L} /$ well secondary antibody solutions for $1.5 \mathrm{~h}$ at room temperature in the dark, washed three times for 10 min with PBS-Tween at room temperature, and filled with $50 \mu \mathrm{L} /$ well of PBS to reduce surface disturbances when scanning. The 800 -channel antibody signals were normalized to the 700-channel signals derived from IR-680 conjugated secondary. Background control wells were prepared by omitting primary antibodies, IRDye $680 \mathrm{CW}$ and IRDye $800 \mathrm{CW}$ (ie secondary only, Li-Cor Biosciences, Lincoln, Nebraska, USA). Plates were scanned and analyzed using an Odyssey ${ }^{\circledR}$ IR CLx system using the Odyssey ${ }^{\circledR}$ imaging software 3.0 (Li-Cor Biosciences). Scan settings were high image quality, $169 \mu \mathrm{m}$ resolution, intensity 6.0 for the 700 -channel, and 6.0 for the 800 -channel with an offset of $4.0 \mathrm{~mm}$. For signal quantification, antibody signals were analyzed as the average 800-channel integrated intensities from duplicate wells normalized to the 700channel signal integrated intensity to correct for well-to-well variations in cell number. Results are expressed as percent inhibition of the NF-kB responses (means \pm standard errors of the mean) compared to vehicle-treated controls.

\section{VI.2.6 Mitochondrial membrane potential}

Active mitochondria with high membrane potential $(\Delta \Psi \mathrm{m})$ accumulate the lipophilic cationic probe 5,5',6,6'-tetrachloro-1,1',3,3'- 
tetraethylbenzimidazolcarbocyanine iodide (JC-1) in aggregates, which are red, whereas, in the mitochondria with low $\Delta \Psi \mathrm{m}$ (inactive), JC-1 stays in a monomeric, green form $[226,227]$. This renders the red:green ratio, a sensitive indicator of the mitochondrial $\Delta \Psi \mathrm{m}$ changes, and indicates cellular ROS production. The change in the mitochondrial membrane potential was measured in fullerene derivative-treated human MC using JC-1 as previously described [228]. Cells $\left(5 \times 10^{5} / 500 \mu 1\right)$ were incubated overnight with fullerene derivative as above, washed, loaded with $2 \mu \mathrm{M} \mathrm{JC}-1$ for $15 \mathrm{~min}$, and activated as above. After cell stimulation, the green fluorescence (the monomeric JC-1) and red fluorescence (JC-1 aggregates) were measured using the FL-1 and FL-2 channels, respectively, with flow cytometry (Becton Dickinson, FACSCalibur ${ }^{\mathrm{TM}}$, East Rutherford, New Jersey, USA).

\section{VI.2.7 Reactive oxygen species measurements}

Mast cells were incubated overnight with fullerene derivatives, washed, and incubated for 30 min with 2',7'-dichlorodihydrofluorescein diacetate (DCF-DA, final concentration of $5 \mu \mathrm{M})$. Next, cells were washed and resuspended in fresh media, placed in a cuvette and activated with IC as above for 50-100 s. ROS fluorescence intensity was measured at the $523 \mathrm{~nm}$ wave length over a 12 minute time interval using spectrophotometry (Perkin Elmer, LS55 Luminescent Spectrometer, Waltham, Massachusetts, USA). All samples were measured in duplicate and performed at least three times. 


\section{VI.2.8 Odyssey imaging of fullerene derivatives in vivo}

To track the fate of fullerene derivatives in vivo an IRDye $800 \mathrm{CW}$ conjugated to ALM using a protocol similar to the one described [21]. These dyes are used in conjunction with the Xenogen imaging system and have been widely used for biodistribution studies [229]. The success of the conjugation and removal of free dye was verified using MALDI-MS (Bruker Corporation, Billerica, Massachusetts, USA) and absorption spectra. Live mice with or without full-blown disease $(\mathrm{K} / \mathrm{BxN}$; day 14$)$ were injected with various concentrations of the ALM-dye and whole body images obtained over $24 \mathrm{~h}$.

\section{VI.2.9 Inflammatory arthritis}

A critical functional role for MC cells in arthritis pathogenesis has been suggested in $\mathrm{K} / \mathrm{BxN}$ serum transfer arthritis [44] while more recent studies using a Kit-independent model for MC-deficiency were fully susceptible to antibody-induced autoimmune arthritis, as Kit mutations affect numerous cell types of both immune and non-immune origin [230]. To this end, Cre-mediated mast cell eradication (Cre-master) mice are used to obviate the deleterious effects associated with Kit mutated mice. To examine the therapeutic effects of ALM (a liposome encapsulated $\mathrm{C}_{70}$ fullerene) and TGA (a watersoluble $\mathrm{C}_{70}$ fullerene conjugated with four glycolic acids), $\mathrm{K} / \mathrm{BxN}$ serum (125 $\left.\mu \mathrm{l}\right)$ was injected intraperitonealy (ip) the day after fullerene derivatives (40 $\mu \mathrm{g} / 100 \mu \mathrm{l} \mathrm{PBS})$ were injected i.p in C57BL/6 or Cre-master mice ( 8 weeks). The next day mice were injected again with serum $(125 \mu \mathrm{l})$. Fullerene derivatives or PBS controls were injected i.p. every 
other day $(40 \mu \mathrm{g} / 100 \mu \mathrm{l}$ PBS) throughout the experiment and the swelling in each ankle was measured using calipers along with the clinical indices as described [44]. After 14 days, serum was collected and assayed for TNF- $\alpha$ levels by ELISA (R\&D systems, Minneapolis, Minnesota, USA). Mice were sacrificed, ankle sections removed, and sections scored as described below. Animal studies for the Cre-Master mice were approved by the Dana Farber Cancer Institute.

To examine fullerene derivatives in the collagen-induced arthritis (CIA) model [231] DBA/1 mice, bovine type II Collagen (CII; MD Bioproducts, St Paul, Minnesota, USA) was dissolved in $10 \mathrm{mM}$ acetic acid at a $4 \mathrm{mg} / \mathrm{ml}$ by stirring overnight at $4^{\circ} \mathrm{C}$, added to an equal volume of complete Freund's adjuvant (Sigma Aldrich, St Louis, Missouri, USA), and homogenized as described [232]. To induce CIA, 8-week-old female DBA/1 inbred mice (Harlan Laboratories, Dublin, Virginia, USA) were injected intradermally at the tail base with $100 \mu \mathrm{l} \mathrm{CII}$ in CFA. Fullerenes (40 $\mu \mathrm{g} / 100 \mu \mathrm{l})$ or PBS were injected i.p. before disease induction and every other day after the first collagen injection. The animals received another injection of CII in CFA at the right hind paw two weeks after the primary immunization. Ankle and paw swelling was measured along with the clinical indices every other day. After four weeks, mice were sacrificed, serum collected, and histochemistry performed on ankles using H\&E staining (IHC World, Woodstock, Maryland, USA). All animal studies for the CIA model were approved by University of North Carolina at Greensboro institutional review board. 


\section{VI.2.10 Disease analysis}

Several parameters of disease were analyzed to determine efficacy of treatment $[44,233]$. The clinical index for each paw/ankle was measured, blinded to treatment group, as follows: $0=$ no evidence of inflammation; $1=$ subtle inflammation (metatarsal phalanges joints, individual phalanx, or localized edema); 2 =easily identified swelling but localized to either dorsal or ventral surface of paw/ankle; and $3=$ swelling on all aspects of paw/ankle. Maximum score $=12$. Quantitative arthritic scores of each mouse (paws and ankles) were measured and expressed as the sum of the measured scores of four limbs. Here, actual swelling of the joint is measured using calipers. The degree of swelling in normal hind limbs (between 120-150 mm) and front limbs (between 110-130 $\mathrm{mm}$ ) is measured every other day starting one day before injections, averaged, and compared statistically to fullerene derivatives treated animals. Histology (H\&E) of paw/ankle sections were analyzed for synovial hyperplasia, pannus formation, and inflammatory cellular infiltrate. Cytokine measurements (TNF- $\alpha$, IL-1) were measured as described [105] using sera from treated and untreated mice.

\section{VI.2.11 Statistics}

Data are presented as mean \pm standard deviation. Analysis of variance (ANOVA) with Newman-Keuls post hoc test was used to compare the effects of fullerene derivatives on mediator release from $\mathrm{MC}$ and on inflammation in murine models with the significance for all tests set at $\mathrm{P}<0.05$. 


\section{VI.3 Results}

VI.3.I The efficacy of fullerene derivative inhibition on inflammatory mediator release and osteoclast formation depends on functional moieties added to the carbon cage

A panel of 40 fullerene derivatives was tested for the ability to inhibit Fce receptor-dependent degranulation and cytokine production from human and mouse MC. Previous studies demonstrated an overnight incubation with $10 \mu \mathrm{g} / \mathrm{ml}$ was optimal for MC stabilization to FceRI-dependent [22] and -independent [234] stimulation and was thus used for these studies. Approximately $15 \%$ of the fullerene derivatives tested significantly $(\mathrm{p}<0.05)$ inhibited both degranulation and IL-1 $\beta$ (Figure 6.1A-C). As demonstrated previously examining Fc\&RI-dependent mediator release [22], several fullerene derivatives exhibited inhibitory capabilities on both degranulation and cytokine production in Fce-stimulated BMMC (Figure 6.1A) and IC-stimulated human tissuederived MC (Figure 6.1B) which was dependent on the side chain moieties added to the carbon cage. Cytokine release from TNF- $\alpha$-challenged synovial fibroblasts was significantly inhibited by $25 \%$ for all fullerene derivatives tested (Figure $6.1 \mathrm{C}$ ). The two most efficacious cytokine blockers (ALM and TGA) also inhibited the formation of bone resorbing osteoclasts (Figure 6.1D). Thus, fullerene derivatives inhibit critical parameters important for the pathologies associated with inflammatory arthritis as assessed by in vitro models. 

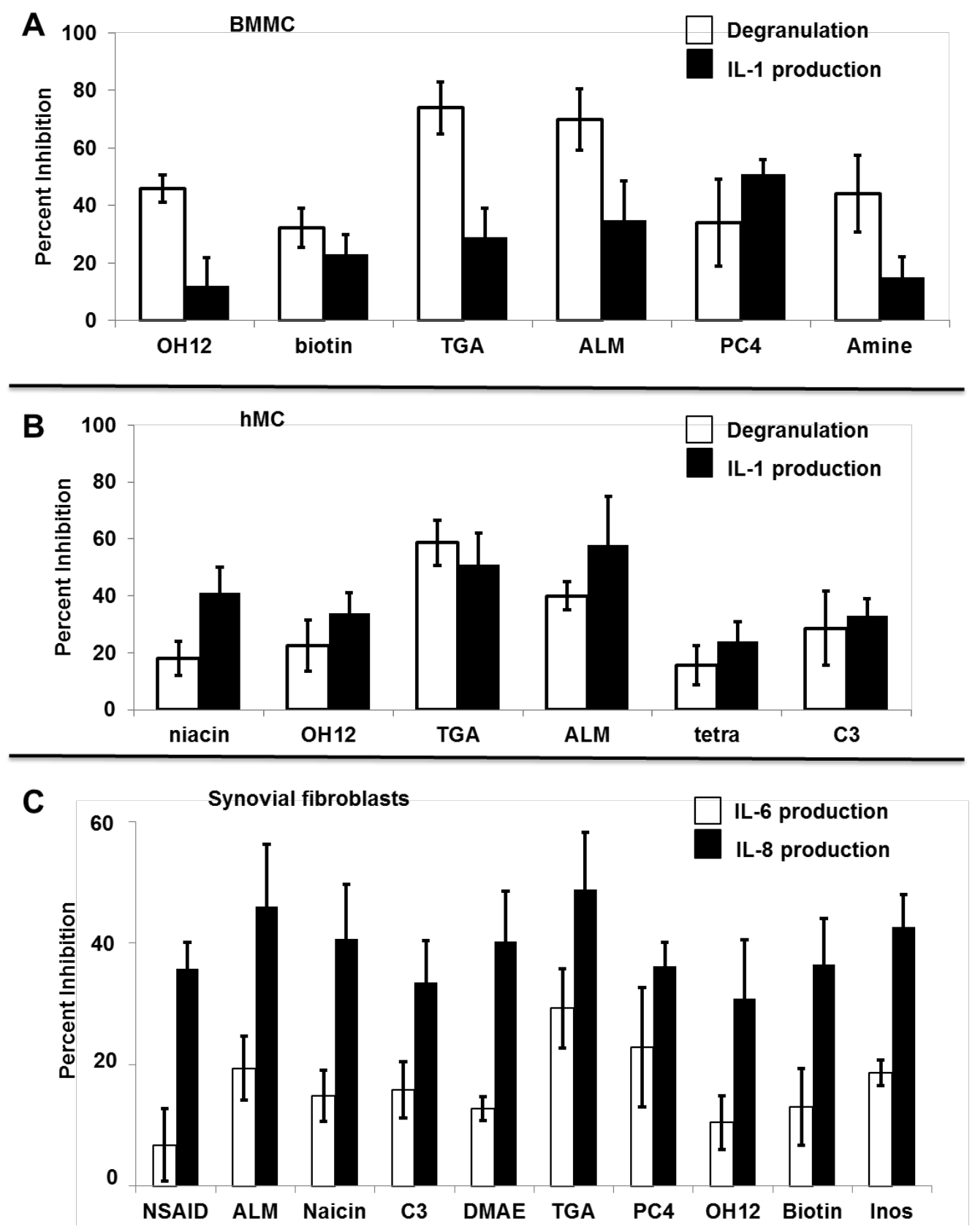

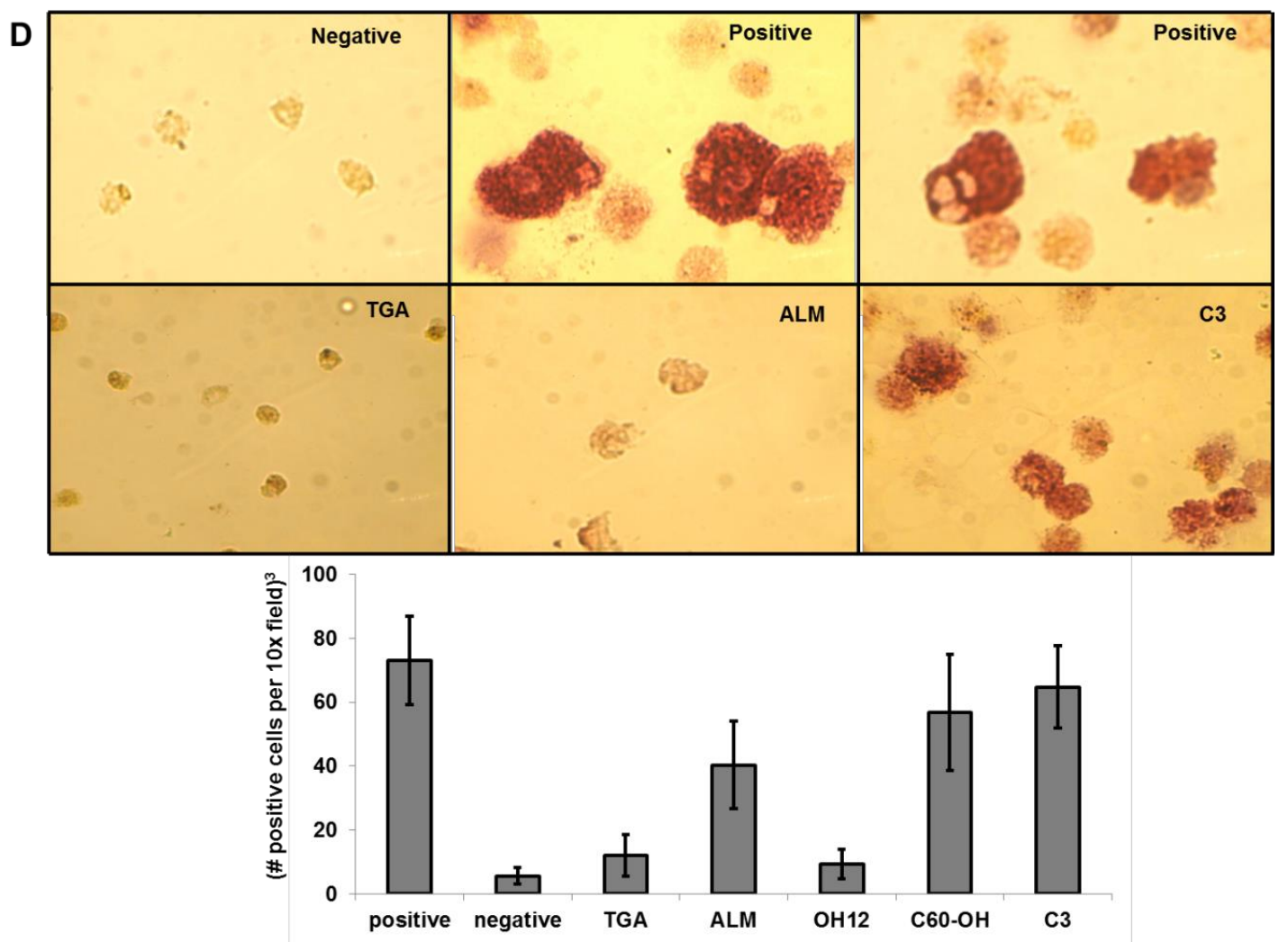

Figure 6.1. Fullerene Derivatives Reduce Degranulation and Cytokine Production from Synovial Fibroblast from RA Patients, Mouse BMMC, and Human MC (hMC), and Osteoclast Formation from Human PBMC. Figure 6.1A shows FceRIInull BMMC incubated with fullerene derivatives overnight $(10 \mu \mathrm{g} / \mathrm{ml})$. The next day anti-FceRII/III antibody $2.4 \mathrm{G} 2$ or isotype control was added followed by crosslinking donkey anti-rat (DAR) F(ab) $)_{2}$. Cells were centrifuged and $\beta$-hexosaminidase release or IL-1 production determined in supernatants or lysates, respectively. Data shown are means \pm SE of triplicate samples that is representative of three experiments. All data was statistically significant with $P$ values $<0.05$. In Figure 6.1B tissue MC were incubated with fullerene derivatives $(10 \mu \mathrm{g} / \mathrm{ml})$ overnight, washed and preformed IgG anti-NP-NP-BSA immune complexes $[8.8 \mu \mathrm{g} / \mathrm{ml}$ anti-NP Ab with $0.13 \mu \mathrm{g} / \mathrm{ml} \mathrm{NP-BSA} \mathrm{[194]],} \mathrm{were} \mathrm{incubated} \mathrm{with} \mathrm{MC} \mathrm{for} 30 \mathrm{~min}$ or $4 \mathrm{~h}$. Supernatants and cell lysates were prepared for mediator release analysis as described. Data is expressed as mean $\pm \mathrm{SE}$ from three individual experiments. $P$ values $<0.05$ by ANOVA when experimental values are compared with the Ab-only control (not shown). Figure 6.1C shows fullerene derivatives can inhibit cytokine production from rheumatoid arthritis-derived synovial fibroblasts. Synovial fibroblasts from RA patients were preincubated with or without various fullerene derivatives $(10 \mu \mathrm{g} / \mathrm{ml})$ overnight, washed, and incubated with TNF- $\alpha(10 \mathrm{ng} / \mathrm{ml}$ for $12 \mathrm{~h})$. Supernatants were saved and cytokines measured in the supernatants. The 
percent inhibition of the treated cells was calculated based on the release of cytokines from non- fullerene derivative treated cells. Figure 6.1D shows the ability

for fullerene derivatives to inhibit osteoclast formation. Human PBMC were incubated without (negative) or with RANK ligand (30 ng/ml) and GMCSF (25 $\mathrm{ng} / \mathrm{ml})$. After $1 \mathrm{~h}$ fullerene derivatives were added $(10 \mu \mathrm{g} / \mathrm{ml})$ and remained throughout. To verify the differentiation of mononuclear cells to osteoclasts, after eight days of culture, cells were analyzed for tartrate resistant acid phosphatase (TRAP) activity by cytochemistry. The cells with the reddish color represent osteoclast formation and are quantified in the graph (bottom). Results are representative of two separate experiments. Magnification 40X.

VI.3.2 Fullerene derivatives inhibit mitochondrial membrane potential, ROS production, and $N F-\kappa B$ activation.

Unstable mitochondrial membrane potential regulates ROS production [235]. Our previous work strongly suggested that fullerene derivatives inhibited degranulation through a pathway involving mitochondrial signaling proteins [20] and ALM is specifically designed to target mitochondrial membranes [48]. However, no studies have examined the role of mitochondrial membrane potential or fullerene derivatives in IC mediator release from human MC. Given that increases in $\mathrm{MC}$ mitochondrial membrane potential closely paralleled degranulation and previous studies suggested mitochondrial signaling pathways were affected by fullerene derivatives, it was hypothesized that the inhibitory effect of fullerene derivatives on MC degranulation may involve modulation of the mitochondrial membrane potential response. Initial studies demonstrated that MC mitochondrial membrane potential was dependent on dose (Figure 6.2A) and time (Figure 6.2B) of the degranulation stimulus using IC. As seen in Figure 6.2C, MC incubated with fullerene derivatives prior to challenge with optimal concentrations of IC demonstrated a decrease in mitochondrial membrane potential compared to untreated MC. ALM and 
TGA also inhibited IC-induced increases in ROS activity (Figure 6.2D). Lastly, NF-kB, which regulates genes controlling the amount of ROS and TNF- $\alpha$ in the cell $[236,237]$, was down-regulated in IC-treated MC pre-incubated with fullerene derivatives (Figure 6.2E). Thus, decreased $\mathrm{MC}$ cellular activation through IC is due in part to decreased mitochondrial membrane potential, ROS production, and NF-kB activation. Two of the overall best inhibitors of these parameters included ALM and TGA, which were chosen for further study.

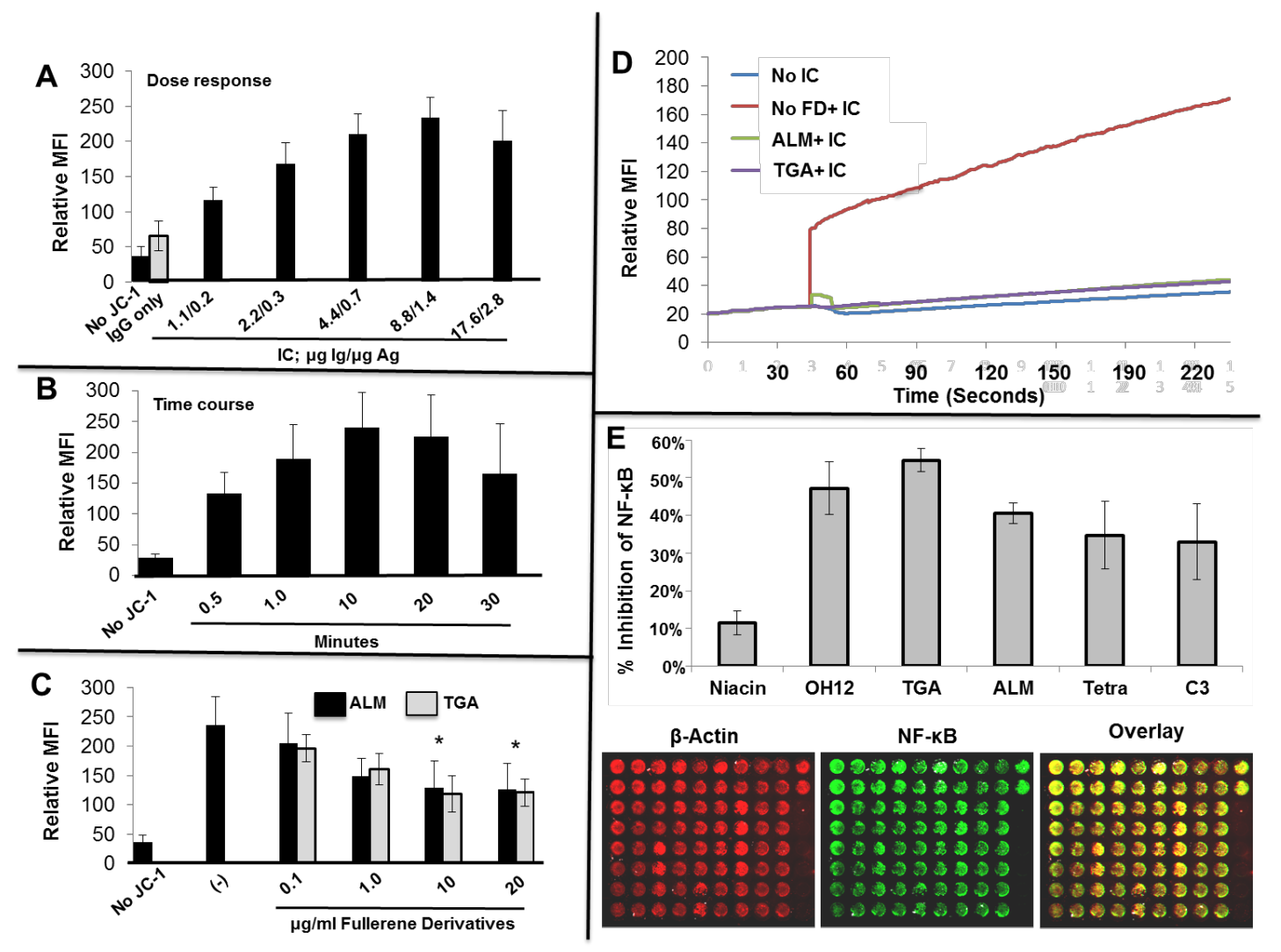

Figure 6.2. Mitochondrial Membrane Potential Correlates with MC Degranulation Through FceR Receptors and is Inhibited by Fullerene Derivatives. In Figure 6.2A, change in mitochondrial membrane potential as a function of the concentration of IC stimulus was assessed. Human MCs were stimulated with graded concentrations of preformed IgG anti-NP/NP-BSA immune complexes as indicated for $10 \mathrm{~min}$. As a 
control, cells without JC-1 and cells with JC-1 plus NP IgG only (no antigen) were incubated in parallel. The above experiment is representative of two separate samples. The percent of degranulation from these cells was $23 \%, 32 \%, 39 \%$, and $45 \%$ respectively. Mitochondrial membrane polarization was quantified by cytofluorimetry (FL2 channel) using FACs analysis as described above. As seen in Figure 6.2B, change in mitochondrial membrane potential as a function of time with fixed concentration of IC stimulus was assessed. Human MCs were stimulated with $8.8 \mu \mathrm{g} / \mathrm{ml}$ anti-NP Ab with $0.13 \mu \mathrm{g} / \mathrm{ml}$ NP-BSA of preformed IgG anti-NP/NP-BSA IC for the indicated times. As a control, cells without JC-1 were incubated in parallel. In Figure 6.2C, Fullerene derivatives inhibit IC-induced increases in mitochondrial membrane potential. Mast cells were incubated overnight with ALM or TGA $(10 \mu \mathrm{g} / \mathrm{ml})$ or media only. The next day cells were challenged with media containing JC-1 probe for $10 \mathrm{~min}$ at $37^{\circ} \mathrm{C}$ with or without IC (as in A). After $10 \mathrm{~min}$ cells were washed with cold PBS, centrifuged and the JC-1 aggregates detected using the FL2. The above experiment is representative of three separate samples. As shown in Figure 6.2D fullerene derivatives inhibit IC-induced elevations in intracellular ROS levels. Mast cells were incubated overnight with fullerene derivatives, washed and DCF-DA added to cells for $30 \mathrm{~min}$ at $37^{\circ} \mathrm{C}$. After washing cells were activated with optimal concentrations of IC and the fluorescence intensity measured at $525 \mathrm{~nm}$ after establishing baseline. Figures show representative numbers from duplicate samples for each condition and are representative of three separate MC cultures. Figure 6.2E shows that fullerene derivatives can block Fce receptor mediated activation of the MC transcription factor NF-KB. Mast cells were incubated with or without fullerene derivatives $(10 \mu \mathrm{g} / \mathrm{ml})$ overnight, washed, and challenged with IC for $24 \mathrm{~h}$. After washing, in-cell Westerns were performed using the manufacturers protocol. Control wells (those without primary antibodies) were reserved as a source for background well intensity. Further controls were cells incubated without fullerene derivatives or IC. Results represent results from two separate experiments.

\section{VI.3.3 ALM targets the inflamed synovial joints, but not organs, in vivo.}

In order to determine the bio-distribution of fullerene derivatives, in vivo experiments were performed using ALM conjugated to an IR-800 dye (ALM-IR-800). As seen in Figure 6.3, at seven days after serum (Figure 6.3A) or vehicle (Figure 6.3B) injection, during the peak symptom scores, ALM is clearly visible six hours post injection in the joints of mice with inflammatory arthritis. In contrast, control mice 
without inflammatory arthritis receiving the same dose of fullerene-dye conjugates did not demonstrate fullerene-dye accumulation in the joints. These data confirm that ALM is capable of migrating and accumulating within the joints of mice with "active" inflammatory arthritis where they are poised to inhibit the inflammatory cascade. Furthermore, organ evaluation (Figure 6.3C) revealed ratios reveal that very little fullerene derivative accumulated non-specifically throughout the body, as quantified in Figure 6.3D. 


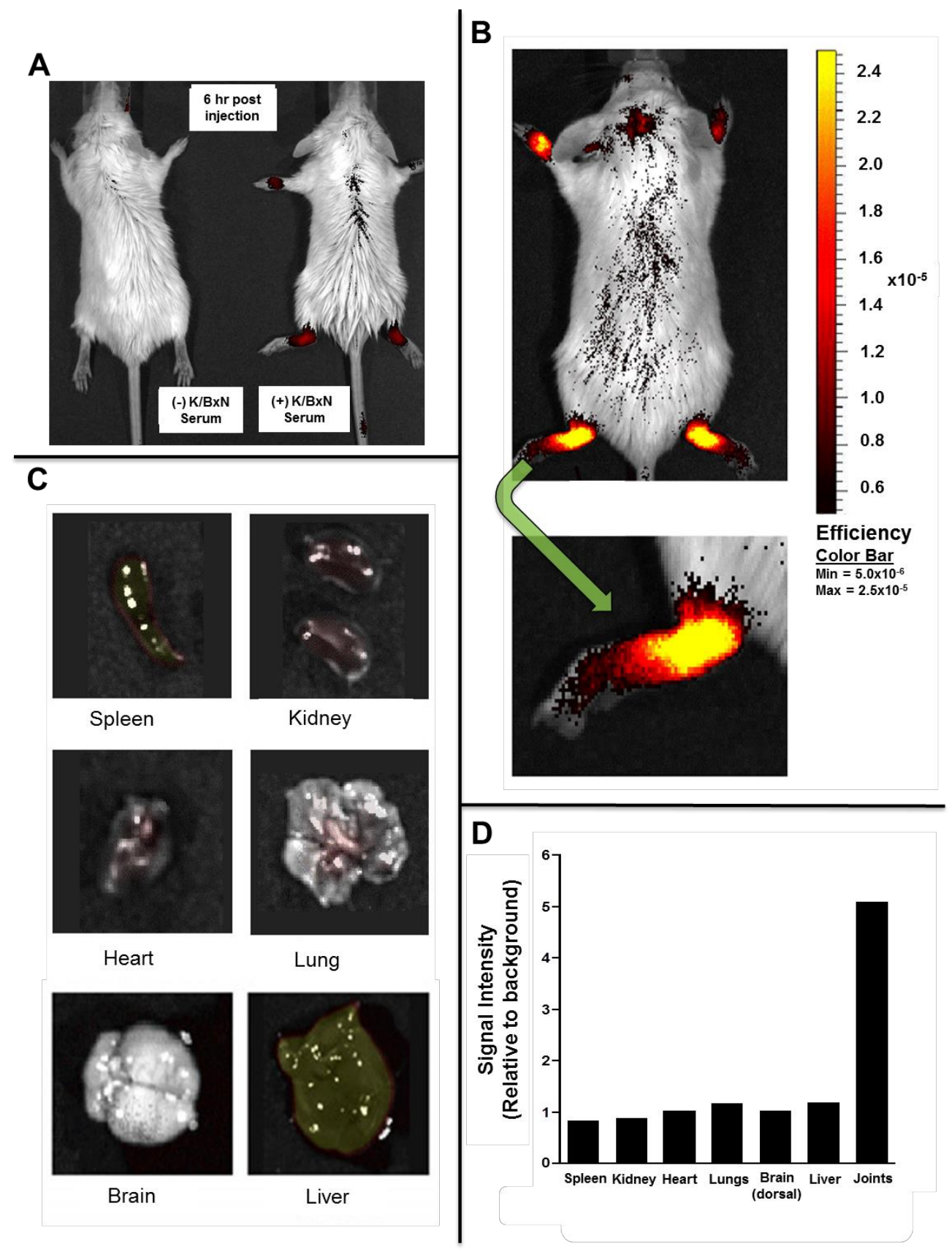

Figure 6.3. ALM Targets Joints in Inflammatory Arthritis. In Figure 6.3A, nonarthritic control (left) and arthritic (right) mice were injected intravenously with 50 $\mu \mathrm{g} / 300 \mu \mathrm{l}$ of ALM-IR800 and imaged $6 \mathrm{~h}$ later using the Odyssey ${ }^{\mathrm{TM}}$ imaging system. Control mice (left) without inflammatory arthritis received the same concentration of fullerene-dye. Note the joint localization of the Dye-fullerene conjugate in the arthritic mouse. Figure 6.3B shows whole mouse imaging and Figure 6.3C shows imaging of externalized organs performed $24 \mathrm{~h}$ after fullerene-dye injection (50 
$\mu \mathrm{g} / 300 \mu \mathrm{l})$. Fluorescence intensity is equally portrayed in all and represent a typical mouse out of three treated in parallel. All of the images have undergone background noise subtraction. Figure 6.3D shows the quantification of fullerene dye concentration in representative organs from the mouse portrayed in Figure 6.3B-C.

\section{VI.3.4 ALM and TGA prevent inflammatory arthritis}

Given our results demonstrating the ability of fullerene derivatives to inhibit MCmediated $[20,22,234]$ and general [15] inflammation, it was hypothesized that fullerene derivatives could inhibit the induction of inflammatory arthritis in vivo. When ALM and TGA were given before disease induction and during the course of disease onset, there was a striking inhibition of the $\mathrm{K} / \mathrm{BxN}$-induced disease pathology in both wild type (C57BL/6) and MC-deficient (Cre-master) mice. After K/BxN serum transfer, PBStreated mice exhibited typical clinical arthritis as determined using clinical indices and quantitative ankle swelling measurements (Figures 6.4A and 6.4B). In contrast, fullerene derivative treated mice demonstrated a significant inhibition in both the clinical indices as well as ankle swelling measurements. Inflammation causing swelling was significantly inhibited at days 9 through 13 in wild-type mice and days 5 through 11 in MC-deficient Cre-master mice. Histochemically, the serum-treated mice demonstrated typical synovial hyperplasia, pannus formation, and inflammatory infiltrates (Figure 64C -top). In contrast, ALM treated animals had less evidence of clinical joint inflammation (Figure 6.4C -middle) which was comparable to non-diseased animals (Figure 6.4C -bottom). 

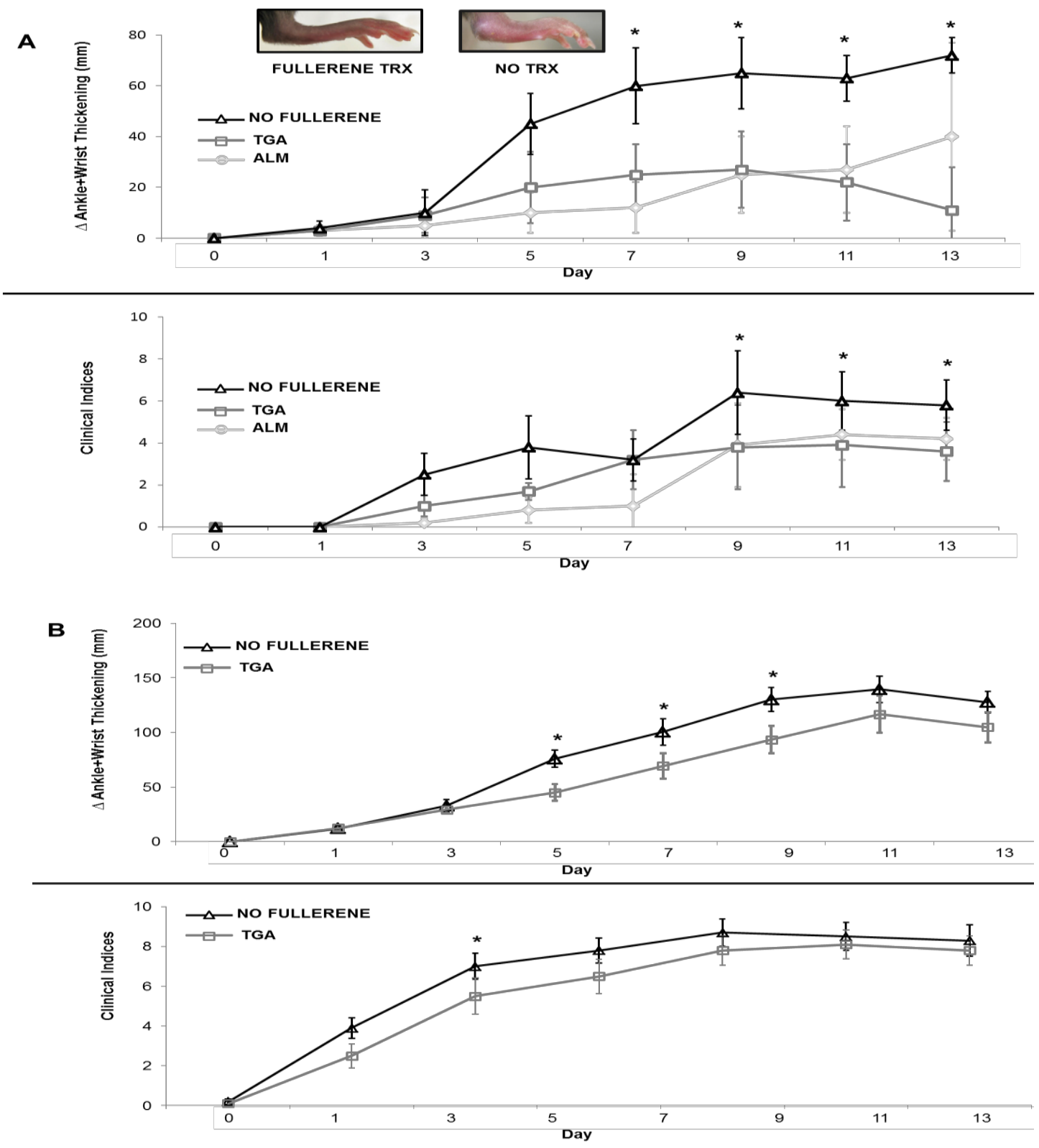
C

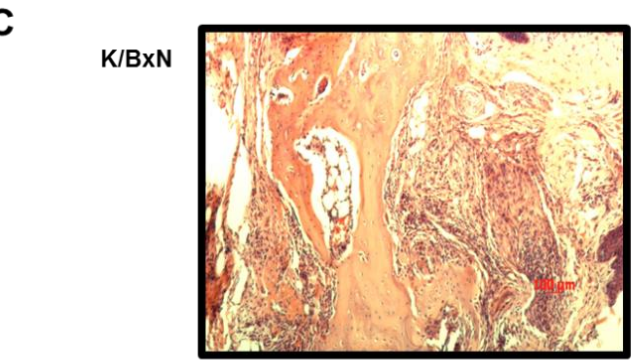

$\mathrm{K} / \mathrm{B} \times \mathrm{N}+\mathrm{ALM}$

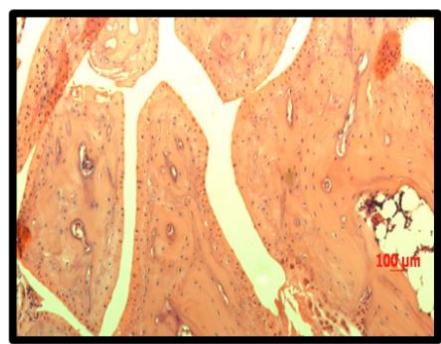

No K/BxN

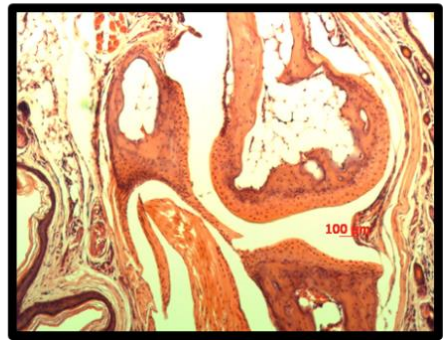

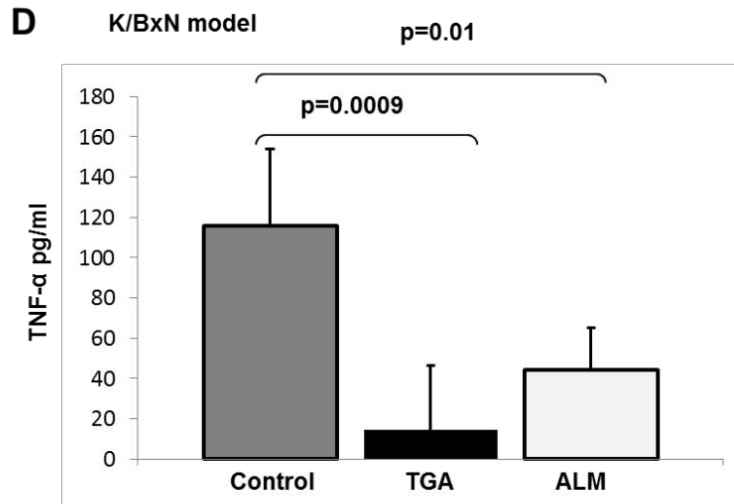

Figure 6.4. Fullerene Derivatives Attenuate Inflammatory Arthritis in the $\mathrm{K} / \mathrm{BxN}$ but not CIA Model. As shown in Figure 6.4A, C57BI/6 (n= 5 mice/group) mice were injected with $\mathrm{K} / \mathrm{BxN}$ serum as described in Methods. TGA or ALM $(40 \mu \mathrm{g} / 100 \mu \mathrm{l})$ were injected i.p. on Day 0,2 , and every second day. As a control $100 \mu \mathrm{l}$ of PBS was injected in the control group. Measurements were taken every second day by a blinded observer. Error bars, \pm SEM. The * indicates significant differences observed on that day in fullerene derivatives compared to non-fullerene-treated mice (see text). Figure 6.4B shows representative ankle sections from $\mathrm{K} / \mathrm{BxN}$ treated mice without ALM (left) or with ALM (middle). Control mice not serum challenged are shown on the right. (Scale bars, $50 \mu \mathrm{m}$ ). Figure 6.4C shows disease pathogenesis

in Cre-Master mice ( $n=10$ mice/group) with and without fullerene derivative therapy. As shown in Figure 6.4D, fullerene derivatives inhibit serum TNF- $\alpha$ levels in the $\mathrm{K} / \mathrm{BxN}$ model and prevent the joint erosion induced by inflammatory arthritis. Serum levels were obtained at peak symptoms (Day 14) from K/BxN models and TNF- $\alpha$ measured as described (CIA model revealed no significant reductions) ( $n=5$ mice per group). 
The CIA model of inflammatory arthritis shares several pathological commonalties with RA, synovial hyperplasia, mononuclear cell infiltration, cartilage degradation, and plays a prominent role in joint destruction [231]. In contrast to the $\mathrm{K} / \mathrm{BxN}$ model there was a small but not significant improvement in the fullerene treated animals compared to untreated animals when measuring ankle thickness and clinical indices (data not shown).

Lastly, the levels of serum TNF- $\alpha$ were significantly lower at day 14 in the mice treated with TGA and ALM compared to those mice given vehicle (PBS) only injection ( $\mathrm{p}=0.0009$ and 0.01 , respectively) in the $\mathrm{K} / \mathrm{BxN}$ model (Figure 6.4D). However, there was no change in the levels of serum TNF- $\alpha$ levels in fullerene derivatives treated and untreated animals in the CIA models (data not shown). These results suggest fullerene derivatives can inhibit inflammatory arthritis and may exert their anti-arthritic inhibitory effect in vivo by blocking TNF- $\alpha$ production.

\section{VI.3.5 Toxicological assessment of ALM and TGA}

In separate experiments high concentrations of ALM and TGA were injected under the same protocol as above except using $100 \mathrm{mg} / \mathrm{kg}$. There was no significant increase in serum activity of ALT and AST between the untreated and ALM and TGAtreated animals indicating no liver toxicity. In fact, both fullerene derivatives provided a small protective effect against the rise in AST/ALT levels in the $\mathrm{K} / \mathrm{BxN}$ and CIA models. Additionally, serum creatinine levels were measured in order to assess kidney toxicity 
[238]. These initial results suggest that ALM and TGA are not acutely toxic to the liver or kidney.

\section{VI.4 Conclusions}

The molecular events leading to the morbidity associated with arthritis is complex and involves a number of factors. Mast cells have been especially implicated in the disease process and preventing mediator release from these cells has been a target for therapeutic intervention $[38,239]$. The initial impetus for these studies was the observation that certain fullerene derivatives can stabilize $\mathrm{MC}$ in vitro and in vivo. Thus, given the potentially important influence of MC on key processes within the chronically inflamed synovium, the hypothesis that fullerene derivatives could ameliorate inflammatory arthritis was tested. It is shown that the efficacy of fullerene derivatives in this process is dependent on how the carbon core is derivatized with side chain moieties. Importantly, no organ accumulation or acute toxicity was detected when assessing liver and kidney function using concentrations twenty times higher than needed for efficacy.

The strategy for these studies was to first determine which fullerene derivatives inhibited human and mouse MC through arthritis-relevant stimulation. In addition, the ability of fullerene derivatives to inhibit synovial fibroblast cytokine production and osteoclast development were considered important prerequisites for predicting in vivo efficacy $[219,240]$. To this end, a panel of fullerene derivatives were tested for their ability to inhibit MC FceR-mediated responses [241]. A clear structure-activity relationship between fullerene derivatives and inhibitory function was not defined. 
However, in general, the fullerene derivatives that were most efficient at inhibiting MC mediator release had side chain moieties that induced maximum water solubility, a zeta potential between 37 and $-146 \mathrm{mV}$, and particle sizes between 50 to $200 \mathrm{nM}$. Of these, both TGA and ALM have been shown previously to inhibit IgE-mediated degranulation and cytokine production [22] and in response to other non-IgE-mediated secretagogues [234]. The TGA (tetra-glycolic acid) is a $\mathrm{C}_{70}$ series with four carboxyl groups, which confers water solubility. It is postulated that the mechanism by which TGA exerts its effect via an interaction between the carboxyl groups and the electrons on the fullerene cage. To examine this point, a similar fullerene derivative that presented a triethylene glycol spacer between the carboxyl groups and the cage was prepared. TEG -TGA (-25 $\mathrm{mV}$ zeta potential; $94 \mathrm{nM}$ particle size) did not block $\mathrm{MC}$ mediator release nor did it interfere with cytokine release (not shown). This result is consistent with the hypothesis that proximity of the carboxyl groups to the cage is necessary for activity.

The mitochondrial electron transport is the machinery that orchestrates one of the most fundamental of chemical processes; the generation of cellular energy from oxygen resulting in the fuel that supports all eukaryotic life. However, it is a highly sensitive process and, unbalanced, leads to the generation of free radicals or ROS which have been linked as a mechanism underlying many chronic human diseases including $\mathrm{MC}$ activation and inflammatory arthritis $[47,242]$. ALM is a mitochondria targeting fullerene derivatives that has been previously shown to home to mitochondria and inhibit inflammation $[15,48]$. ALM was designed to accumulate in the internal mitochondrial membrane bilayers positioned to neutralize superoxide molecules, reactive lipid radicals, 
and radicals that have formed on transmembrane proteins at the site where they are generated. Subsequently, this is predicted to impact diseases whose pathologies stem from radical injury.

To this end both fullerene derivatives significantly block ROS production and mitochondrial membrane potential. While it has been shown previously that human MC degranulation in response to FceRI and Fce-signaling involves ROS [58,59], it is not clear if blocking ROS directly blocks degranulation and cytokine production. Results here suggest that blocking ROS using ALM and TGA in response to IC (an FceRIIAdependent stimuli [194]) parallels inhibition of mediator release. This is in line with previous work suggesting that fullerenes interfere with the generation of mitochondrialderived $\operatorname{ROS}[51,52,243,244]$. It is also demonstrated that mitochondrial membrane potential is a critical determinant in human MC FceR-mediated degranulation. While further studies are needed these data suggest that fullerenes inhibit MC through a mechanism involving the mitochondrial membrane potential and suggest a critical role of the mitochondria in human MC non-IgE mediator release.

Nuclear factor-kappa B is involved in the pathophysiology of inflammatory and efforts to target its function through molecular targets in the pathway leading to its activation are underway [245-247]. This transcription factor induces both TNF- $\alpha$ and IL$1 \beta$ gene expression which can both in turn activate the NF-kB pathway inducing an autocrine loop which perpetuates inflammation. Interestingly, some of the drugs for RA were shown to block either the NF-kB activation cascade or its action [245, 247, 248]. For example, gold-containing therapeutics, TNF- $\alpha$ inhibitors, and methotrexate, all 
regularly used for treating arthritis, can effect NF-kB function [249, 250]. Several fullerene derivatives, including ALM and TGA, inhibited IC-induced NF-kB activation in human MC. Current studies are examining what signaling molecules in the ROS/TNF/NF-kB pathway [237] are affected by fullerene derivatives.

Arthritic joint tissues demonstrate a striking predilection for uptake of ALM. Indeed, this strong uptake may provide the basis of their efficacy in ameliorating arthritis. It was also demonstrated that fullerene derivatives inhibited the onset of arthritis in $\mathrm{K} / \mathrm{BxN}$ serum transfer arthritis in $\mathrm{C} 57 \mathrm{Bl} / 6$ mice. However, there was a small but not significant improvement in the CIA model. The $\mathrm{K} / \mathrm{BxN}$ model induces a rapid and severe synovitis dependent on neutrophils, $\mathrm{MC}$, and macrophages. A role for $\mathrm{MC}$ was revealed from studies in which two strains of mice deficient in this cell type, W/Wv and Sl/Sld, were resistant to disease induction following serum transfer [44]. Susceptibility to disease is restored in the $\mathrm{W} / \mathrm{Wv}$ strain by MC engraftment. However, new research [230] has revealed that the $\mathrm{K} / \mathrm{BxN}$ model, is not $\mathrm{MC}$-dependent as the original findings may be a result of Kit mutations affecting multiple cell types. In contrast, the Cre-master model ablates $\mathrm{MC}$ numbers through a different mechanism: by targeted insertion of Crerecombinase into the $\mathrm{MC}$ carboxypeptidase $\mathrm{A} 3$ locus and subsequent Trp53-dependent MC depletion. Cre-mediated mice are fully susceptible to antibody-induced autoimmune arthritis. This suggests the inhibition seen with fullerenes possibly occurs due to the blocking of some component associated with the $\mathrm{K} / \mathrm{BxN}$ serum transfer as this is the major difference in the two models suggesting a more robust and promiscuous pathway in which the fullerenes are reducing disease symptoms. Taken together, our results 
suggest that fullerene derivatives block arthritis in certain mouse models that blocks some component of TNF- $\alpha$ release which may or may not include MC activation. Given the vast differences in MC phenotypes and expression in the rodent vs. human systems [118] there may also be differences in the ability of fullerenes to inhibit MC from the two species. Further studies are needed to determine whether fullerenes may be a valid strategy for human arthritis intervention.

As in other studies using purified and well characterized fullerene derivatives [49, 198, 199] no liver or kidney toxicity was detected using repeated dosing of concentrations higher than that needed for in vivo efficacy [177]. The in vivo imaging studies also demonstrated a lack of uptake in other organs, which portends well for a favorable toxicity profile in clinical development of ALM. Of course, more advanced toxicity studies are needed to assess these two fullerene derivatives as is the case with any new therapeutic.

In conclusion, it was demonstrated that not all fullerene derivatives exhibit the same ability to inhibit inflammatory mediator release from MC and synovial fibroblasts. Two fullerene derivatives were able to significantly block the onset of serum-induced arthritis in vivo leading to a blunted inflammatory response; however CIA-induced mice were refractory to fullerene treatment. More studies are needed to identify those structure activity relationships that are dependent on the moieties added to the fullerene carbon cage in order to define the precise mechanism by which these fullerene derivatives inhibit inflammatory disease. 


\section{CHAPTER VII}

\section{FUTURE PERSPECTIVES}

Fullerenes and their unique properties can be beneficial to many modern medical applications; as they are composed entirely of carbon in a hollow sphere (without requiring a toxic metal catalyst), their functionalization (with chemicals to increase their hydrophilicity) or encapsulation (in carriers, i.e., as liposomes) allows for easy solubility in biologically suitable aqueous media. The innate properties of fullerenes render high antioxidant capacity at extremely small sizes that can be functionalized or tailored to accommodate a broad range of diagnostic and therapeutic applications. Recent developments and future research in the fullerene field could reveal numerous commercial applications spanning a broad spectrum: antiviral; anticancer; photosensitizers; antioxidant activity; drug delivery and gene therapy; and highly sensitivetargeted diagnostic applications.

Current research in fullerenes could create significant strides in slowing or suppressing the progression of HIV infection to AIDS [251-253]. Mechanistically, their success as a potential antiviral application is based on the compounds unique geometry and high antioxidant activity. The compounds are capable of complexing and inhibiting the HIV protease with a relatively high structure-activity relationship [251]. The fullerenes can spatially bind in the cavity ( $10 \AA$ approximate diameter) regions of HIV protease tightly and prevent the release of water, subsequently inhibiting the active 
regions of the protease [254]. The fullerenes strong affinity to bind to the enzymes active site results in significant inhibition of viral replication [252, 255].

Fullerenes are also capable of being photoexcited and have, thus, been exploited as photosensitizers, whereby fullerenes can be conjugated with high affinity to specific proteins and nucleic acids. The photoirradiated fullerene is excited from its ground state and converted back via intersystem crossing, creating a highly toxic singlet oxygen species. Researchers have demonstrated that using photodynamic therapy, fullerenes could photosensitize specifically targeted cells, which could rapidly reduce cell viability [256]. This technique has also been modified to a theranostic approach utilizing gadolinium-containing endohedral fullerenes to selectively target, photosensitize and image tumor reduction over time [257].

The potential for using fullerene-based medicines is substantial but concerns of toxicity have slowed the initial enthusiasm that surrounded their discovery. Only those studies using well-characterized, single species, 'lead candidate' fullerene formulations can provide meaningful information regarding potential toxicological effects. Such studies are needed as the state of research today with fullerenes is shaped by studies such as these that address the observation "that extrapolation across similar nanoparticles will be dependent upon surface chemistry and concentration which may affect the degree of agglomeration and thus biological effects" [258]. Thus, more thorough studies will serve as a building block in developing a database that links surface functionalization chemistry of fullerene compounds to biological function. Significant strides have been made throughout the last decade to help propel fullerenes as a functional platform to help solve 
many modern medical limitations. However, the translation of successful research into market application will require more work to better understand uptake, biodistribution, absorption, lifetime, excretion and, ultimately, consumer safety of these nanostructures.

The unique physical and chemistry properties, the decrease in production cost, increased scalability and broad range of potential medical applications have stimulated a lot of research within the industry. While initial toxicity concerns stalled progress, recent studies have shown limited evidence of any toxicity associated with these novel nanomaterials. In time the fullerene field will make strides in penetrating industry, but only when more thorough characterization and sufficient research is accomplished to address long-term, reproducible toxicological data will this fascinating class of molecules bring advances to modern medicine. 


\section{REFERENCES}

1. Rao, C.N.R. and A.K. Cheetham, Science and technology of nanomaterials: current status and future prospects. Journal of Materials Chemistry, 2001. 11(12): p. 2887-2894.

2. Liu, Z., et al., Carbon materials for drug delivery and cancer therapy. Materials Today, 2011. 14(7-8): p. 316-323.

3. Lu, F., et al., Advances in Bioapplications of Carbon Nanotubes. Advanced Materials, 2009. 21(2): p. 139-152.

4. $\quad$ Sun, Z., et al., Carbon nanotubes enhance cytotoxicity mediated by human lymphocytes in vitro. PLoS One, 2011. 6(6): p. e21073.

5. $\quad$ Shen, H., et al., Biomedical applications of graphene. Theranostics, 2012. 2(3): p. 28394.

6. Feng, L., S. Zhang, and Z. Liu, Graphene based gene transfection. Nanoscale, 2011. 3(3): p. 1252-7.

7. Basso, A.S., et al., Reversal of axonal loss and disability in a mouse model of progressive multiple sclerosis. J Clin Invest, 2008. 118(4): p. 1532-43.

8. $\quad$ Dugan, L.L., et al., Carboxyfullerenes as neuroprotective agents. Proc Natl Acad Sci U S A, 1997. 94(17): p. 9434-9.

9. Bosi, S., et al., Synthesis and anti-HIV properties of new water-soluble bisfunctionalized[60]fullerene derivatives. Bioorg Med Chem Lett, 2003. 13(24): p. 4437-40.

10. Marchesan, S., et al., Anti-HIV properties of cationic fullerene derivatives. Bioorg Med Chem Lett, 2005. 15(15): p. 3615-8.

11. Berger, C.S., et al., Cell Internalization Studies of Gadofullerene-(ZME-018) Immunoconjugates into A375m Melanoma Cells. Transl Oncol, 2011. 4(6): p. 350-4.

12. Daroczi, B., et al., In vivo radioprotection by the fullerene nanoparticle DF-1 as assessed in a zebrafish model. Clin Cancer Res, 2006. 12(23): p. 7086-91.

13. Lai, Y.L., P. Murugan, and K.C. Hwang, Fullerene derivative attenuates ischemiareperfusion-induced lung injury. Life Sci, 2003. 72(11): p. 1271-8.

14. Gonzalez, K.A., et al., Synthesis and in vitro characterization of a tissue-selective fullerene: vectoring $\mathrm{C}(60)(\mathrm{OH})(16) A M B P$ to mineralized bone. Bioorg Med Chem, 2002. 10(6): p. 1991-7.

15. Dellinger, A., et al., Fullerene nanomaterials inhibit phorbol myristate acetate-induced inflammation. Exp Dermatol, 2009. 18(12): p. 1079-81.

16. Tsao, N., et al., In vitro action of carboxyfullerene. J Antimicrob Chemother, 2002. 49(4): p. 641-9.

17. Quick, K.L., et al., A carboxyfullerene SOD mimetic improves cognition and extends the lifespan of mice. Neurobiol Aging, 2008. 29(1): p. 117-28.

18. Baati, T., et al., The prolongation of the lifespan of rats by repeated oral administration of [60]fullerene. Biomaterials, 2012. 33(19): p. 4936-46.

19. Yamago, S., et al., In vivo biological behavior of a water-miscible fullerene: 14C labeling, absorption, distribution, excretion and acute toxicity. Chem Biol, 1995. 2(6): p. 385-9.

20. Ryan, J.J., et al., Fullerene nanomaterials inhibit the allergic response. J Immunol, 2007. 179(1): p. 665-72. 
21. Dellinger, A., et al., Uptake and distribution of fullerenes in human mast cells.

Nanomedicine, 2010. 6(4): p. 575-82.

22. Norton, S.K., et al., A new class of human mast cell and peripheral blood basophil stabilizers that differentially control allergic mediator release. Clin Transl Sci, 2010. 3(4): p. 158-69.

23. Galli, S.J., M. Tsai, and A.M. Piliponsky, The development of allergic inflammation. Nature, 2008. 454(7203): p. 445-54.

24. Kobayashi, T., et al., An essential role of mast cells in the development of airway hyperresponsiveness in a murine asthma model. J Immunol, 2000. 164(7): p. 3855-61.

25. Williams, C.M. and S.J. Galli, Mast cells can amplify airway reactivity and features of chronic inflammation in an asthma model in mice. J Exp Med, 2000. 192(3): p. 455-62.

26. Gordon, J.R. and S.J. Galli, Mast cells as a source of both preformed and immunologically inducible TNF-alpha/cachectin. Nature, 1990. 346(6281): p. 274-6.

27. Brightling, C., M. Berry, and Y. Amrani, Targeting TNF-alpha: a novel therapeutic approach for asthma. J Allergy Clin Immunol, 2008. 121(1): p. 5-10; quiz 11-2.

28. Kepley, C.L., et al., Immunohistochemical detection of human basophils in postmortem cases of fatal asthma. Am J Respir Crit Care Med, 2001. 164(6): p. 1053-8.

29. Kepley, C.L., S.S. Craig, and L.B. Schwartz, Identification and partial characterization of a unique marker for human basophils. J Immunol, 1995. 154(12): p. 6548-55.

30. Schroeder, J.T., Basophils beyond effector cells of allergic inflammation. Adv Immunol, 2009. 101: p. 123-61.

31. Jackson, D.J., et al., Asthma exacerbations: origin, effect, and prevention. J Allergy Clin Immunol, 2011. 128(6): p. 1165-74.

32. Norton, S.K., et al., Epoxyeicosatrienoic acids are involved in the C(70) fullerene derivative-induced control of allergic asthma. J Allergy Clin Immunol, 2012. 130(3): p. 761-769 e2.

33. Sudhahar, V., S. Shaw, and J.D. Imig, Epoxyeicosatrienoic acid analogs and vascular function. Curr Med Chem, 2010. 17(12): p. 1181-90.

34. Pfister, S.L., K.M. Gauthier, and W.B. Campbell, Vascular pharmacology of epoxyeicosatrienoic acids. Adv Pharmacol, 2010. 60: p. 27-59.

35. Morin, C. and E. Rousseau, Effects of 5-oxo-ETE and 14,15-EET on reactivity and Ca2+ sensitivity in guinea pig bronchi. Prostaglandins Other Lipid Mediat, 2007. 82(1-4): p. 30-41.

36. Morin, C., et al., EET displays anti-inflammatory effects in TNF-alpha stimulated human bronchi: putative role of CPI-17. Am J Respir Cell Mol Biol, 2008. 38(2): p. 192-201.

37. Node, K., et al., Anti-inflammatory properties of cytochrome P450 epoxygenase-derived eicosanoids. Science, 1999. 285(5431): p. 1276-9.

38. Nigrovic, P.A. and D.M. Lee, Synovial mast cells: role in acute and chronic arthritis. Immunol Rev, 2007. 217: p. 19-37.

39. Woolley, D.E., The mast cell in inflammatory arthritis. N Engl J Med, 2003. 348(17): p. 1709-11.

40. Irani, A., et al., Distribution of mast cell subsets in rheumatoid arthritis and osteoarthritis Arthritis Rheum. , 1987. 34: p. 1116-1124.

41. Bridges, A.J., et al., Human synovial mast cell involvement in rheumatoid arthritis and osteoarthritis. Relationship to disease type, clinical activity, and antirheumatic therapy. Arthritis Rheum, 1991. 34(9): p. 1116-24.

42. Gotis-Graham, I., et al., Synovial mast cell responses during clinical improvement in early rheumatoid arthritis. Ann Rheum Dis, 1998. 57(11): p. 664-71. 
43. Lavery, J.P. and J.R. Lisse, Preliminary study of the tryptase levels in the synovial fluid of patients with inflammatory arthritis. Ann Allergy, 1994. 72(5): p. 425-7.

44. Lee, D.M., et al., Mast cells: a cellular link between autoantibodies and inflammatory arthritis. Science, 2002. 297(5587): p. 1689-92.

45. Dinser, R., Animal models for arthritis. Best Pract Res Clin Rheumatol, 2008. 22(2): p. 253-67.

46. Nigrovic, P.A. and D.M. Lee, Mast cells in autoantibody responses and arthritis. Novartis Found Symp, 2005. 271: p. 200-9; discussion 210-4.

47. Winyard, P.G., et al., Measurement and meaning of markers of reactive species of oxygen, nitrogen and sulfur in healthy human subjects and patients with inflammatory joint disease. Biochem Soc Trans, 2011. 39(5): p. 1226-32.

48. Zhou, Z., et al., Liposomal formulation of amphiphilic fullerene antioxidants. Bioconjug Chem, 2010. 21(9): p. 1656-61.

49. Ehrich, M., et al., Fullerene antioxidants decrease organophosphate-induced acetylcholinesterase inhibition in vitro. Toxicol In Vitro, 2011. 25(1): p. 301-7.

50. Zhao, W., et al., In vitro desensitization of human skin mast cells. J Clin Immunol, 2012. 32(1): p. 150-60.

51. Chirico, F., et al., Carboxyfullerenes localize within mitochondria and prevent the UVBinduced intrinsic apoptotic pathway. Exp Dermatol, 2007. 16(5): p. 429-36.

52. Fumelli, C., et al., Carboxyfullerenes protect human keratinocytes from ultraviolet-Binduced apoptosis. J Invest Dermatol, 2000. 115(5): p. 835-41.

53. Pitman, N., et al., Collagen-induced arthritis is not impaired in mast cell-deficient mice. Ann Rheum Dis, 2011. 70(6): p. 1170-1.

54. Brown, M.A., M.B. Tanzola, and M. Robbie-Ryan, Mechanisms underlying mast cell influence on EAE disease course. Mol Immunol, 2002. 38(16-18): p. 1373-8.

55. Gregory, G.D., et al., MASTering the immune response: mast cells in autoimmunity. Novartis Found Symp, 2005. 271: p. 215-25; discussion 225-31.

56. Secor, V.H., et al., Mast cells are essential for early onset and severe disease in a murine model of multiple sclerosis. J Exp Med, 2000. 191(5): p. 813-22.

57. Li, L.B. and M.E. Reith, Modeling of the interaction of $\mathrm{Na}+$ and $\mathrm{K}+$ with the binding of dopamine and [3H]WIN 35,428 to the human dopamine transporter. J Neurochem, 1999. 72(3): p. 1095-109.

58. Dugan, L.L., et al., Buckminsterfullerenol free radical scavengers reduce excitotoxic and apoptotic death of cultured cortical neurons. Neurobiol Dis, 1996. 3(2): p. 129-35.

59. Ali, S.S., et al., A biologically effective fullerene (C60) derivative with superoxide dismutase mimetic properties. Free Radic Biol Med, 2004. 37(8): p. 1191-202.

60. Borland, M.K., et al., Chronic, low-dose rotenone reproduces Lewy neurites found in early stages of Parkinson's disease, reduces mitochondrial movement and slowly kills differentiated SH-SY5Y neural cells. Mol Neurodegener, 2008. 3: p. 21.

61. Gilgun-Sherki, Y., E. Melamed, and D. Offen, The role of oxidative stress in the pathogenesis of multiple sclerosis: the need for effective antioxidant therapy. J Neurol, 2004. 251(3): p. 261-8.

62. ClinicalTrials.gov. Available from: http://clinicaltrials.gov/.

63. Bakry, R., et al., Medicinal applications of fullerenes. Int J Nanomedicine, 2007. 2(4): p. 639-49.

64. Mody, V.V., M.I. Nounou, and M. Bikram, Novel nanomedicine-based MRI contrast agents for gynecological malignancies. Adv Drug Deliv Rev, 2009. 61(10): p. 795-807. 
65. Nitta, N., et al., Is the use of fullerene in photodynamic therapy effective for atherosclerosis? Cardiovasc Intervent Radiol, 2008. 31(2): p. 359-66.

66. Bolskar, R.D., Gadofullerene MRI contrast agents. Nanomedicine (Lond), 2008. 3(2): p. 201-13.

67. Chewning, R.H. and K.J. Murphy, Gadolinium-based contrast media and the development of nephrogenic systemic fibrosis in patients with renal insufficiency. J Vasc Interv Radiol, 2007. 18(3): p. 331-3.

68. Runge, V.M., Gadolinium and nephrogenic systemic fibrosis. AJR Am J Roentgenol, 2009. 192(4): p. W195-6; discussion W197.

69. Ledneva, E., et al., Renal safety of gadolinium-based contrast media in patients with chronic renal insufficiency. Radiology, 2009. 250(3): p. 618-28.

70. Cowper, S.E., Nephrogenic systemic fibrosis: a review and exploration of the role of gadolinium. Adv Dermatol, 2007. 23: p. 131-54.

71. MacFarland, D.K., et al., Hydrochalarones: a novel endohedral metallofullerene platform for enhancing magnetic resonance imaging contrast. J Med Chem, 2008. 51(13): p. 3681-3.

72. Ouimet, T., et al., Molecular and cellular targets of the MRI contrast agent P947 for atherosclerosis imaging. Mol Pharm, 2012. 9(4): p. 850-61.

73. Uno, K. and S.J. Nicholls, Biomarkers of inflammation and oxidative stress in atherosclerosis. Biomark Med, 2010. 4(3): p. 361-73.

74. Sadeghi, M.M., et al., Imaging atherosclerosis and vulnerable plaque. J Nucl Med, 2010. 51 Suppl 1: p. 51S-65S.

75. Nergiz-Unal, R., et al., CD36 as a multiple-ligand signaling receptor in atherothrombosis. Cardiovasc Hematol Agents Med Chem, 2011. 9(1): p. 42-55.

76. Collot-Teixeira, S., et al., CD36 and macrophages in atherosclerosis. Cardiovasc Res, 2007. 75(3): p. 468-77.

77. Ge, Y. and M.T. Elghetany, CD36: a multiligand molecule. Lab Hematol, 2005. 11(1): p. $31-7$.

78. Silverstein, R.L. and M. Febbraio, CD36, a scavenger receptor involved in immunity, metabolism, angiogenesis, and behavior. Sci Signal, 2009. 2(72): p. re3.

79. Podrez, E.A., et al., Identification of a novel family of oxidized phospholipids that serve as ligands for the macrophage scavenger receptor CD36. J Biol Chem, 2002. 277(41): p. 3850316.

80. Kolovou, G., et al., Apolipoprotein E knockout models. Curr Pharm Des, 2008. 14(4): p. 338-51.

81. Patra, C.R., et al., A core-shell nanomaterial with endogenous therapeutic and diagnostic functions. Cancer Nanotechnol, 2010. 1(1): p. 13-18.

82. Yigit, M.V., et al., Noninvasive MRI-SERS imaging in living mice using an innately bimodal nanomaterial. ACS Nano, 2011. 5(2): p. 1056-66.

83. Park, J.H., et al., Micellar hybrid nanoparticles for simultaneous magnetofluorescent imaging and drug delivery. Angew Chem Int Ed Engl, 2008. 47(38): p. 7284-8.

84. Park, J.H., et al., Magnetic Iron Oxide Nanoworms for Tumor Targeting and Imaging. Adv Mater, 2008. 20(9): p. 1630-1635.

85. von Maltzahn, G., et al., In vivo tumor cell targeting with "click" nanoparticles. Bioconjug Chem, 2008. 19(8): p. 1570-8.

86. Jaffer, F.A., P. Libby, and R. Weissleder, Molecular and cellular imaging of atherosclerosis: emerging applications. J Am Coll Cardiol, 2006. 47(7): p. 1328-38. 
87. Jaffer, F.A., et al., Cellular imaging of inflammation in atherosclerosis using magnetofluorescent nanomaterials. Mol Imaging, 2006. 5(2): p. 85-92.

88. McCarthy, J.R., F.A. Jaffer, and R. Weissleder, A macrophage-targeted theranostic nanoparticle for biomedical applications. Small, 2006. 2(8-9): p. 983-7.

89. Li, C., et al., Targeting glioblastoma multiforme with an IL-13/diphtheria toxin fusion protein in vitro and in vivo in nude mice. Protein Eng, 2002. 15(5): p. 419-27.

90. Todhunter, D.A., et al., A bispecific immunotoxin (DTAT13) targeting human IL-13 receptor $(I L-13 R)$ and urokinase-type plasminogen activator receptor (uPAR) in a mouse xenograft model. Protein Eng Des Sel, 2004. 17(2): p. 157-64.

91. Madhankumar, A.B., et al., Interleukin-13 receptor-targeted nanovesicles are a potential therapy for glioblastoma multiforme. Mol Cancer Ther, 2006. 5(12): p. 3162-9.

92. Oberdorster, E., Manufactured nanomaterials (fullerenes, C60) induce oxidative stress in the brain of juvenile largemouth bass. Environ Health Perspect, 2004. 112(10): p. 1058-62.

93. Barnaby, J., Study raises concerns about carbon particles in New York Times 2004.

94. Zhu, S., E. Oberdorster, and M.L. Haasch, Toxicity of an engineered nanoparticle (fullerene, C60) in two aquatic species, Daphnia and fathead minnow. Mar Environ Res, 2006. 62 Suppl: p. S5-9.

95. Henry, T.B., E.J. Petersen, and R.N. Compton, Aqueous fullerene aggregates (nC60) generate minimal reactive oxygen species and are of low toxicity in fish: a revision of previous reports. Curr Opin Biotechnol, 2011. 22(4): p. 533-7.

96. US FDA. Nanotechnology. Available from: http://fda.gov/scienceresearch.

97. National Cancer Institute. Nanotechnology Characterization Laboratory Objectives. .

98. Chen, H.H., et al., Acute and subacute toxicity study of water-soluble polyalkylsulfonated C60 in rats. Toxicol Pathol, 1998. 26(1): p. 143-51.

99. Mori, T., et al., Preclinical studies on safety of fullerene upon acute oral administration and evaluation for no mutagenesis. Toxicology, 2006. 225(1): p. 48-54.

100. Gharbi, N., et al., [60]fullerene is a powerful antioxidant in vivo with no acute or subacute toxicity. Nano Lett, 2005. 5(12): p. 2578-85.

101. Kalesnikoff, J. and S.J. Galli, New developments in mast cell biology. Nat Immunol, 2008. 9(11): p. 1215-23.

102. Stone, K.D., C. Prussin, and D.D. Metcalfe, IgE, mast cells, basophils, and eosinophils. J Allergy Clin Immunol, 2010. 125(2 Suppl 2): p. S73-80.

103. Kovanen, P.T., Mast cells: multipotent local effector cells in atherothrombosis. Immunol Rev, 2007. 217: p. 105-22.

104. Djordjevic, A., G. Bogdanovic, and S. Dobric, Fullerenes in biomedicine. J BUON, 2006. 11(4): p. 391-404.

105. Kepley, C.L., Antigen-induced reduction in mast cell and basophil functional responses due to reduced Syk protein levels. Int Arch Allergy Immunol, 2005. 138(1): p. 29-39.

106. Kepley, C.L., et al., Co-aggregation of FcgammaRII with FcepsilonRI on human mast cells inhibits antigen-induced secretion and involves SHIP-Grb2-Dok complexes. J Biol Chem, 2004. 279(34): p. 35139-49.

107. Vonakis, B.M., et al., Basophil FcepsilonRI histamine release parallels expression of Src-homology 2-containing inositol phosphatases in chronic idiopathic urticaria. J Allergy Clin Immunol, 2007. 119(2): p. 441-8.

108. Tkaczyk, C., D.D. Metcalfe, and A.M. Gilfillan, Determination of protein phosphorylation in Fc epsilon RI-activated human mast cells by immunoblot analysis requires protein extraction under denaturing conditions. J Immunol Methods, 2002. 268(2): p. 239-43. 
109. Kulka, M., et al., Neuropeptides activate human mast cell degranulation and chemokine production. Immunology, 2008. 123(3): p. 398-410.

110. Church, M.K., G.J. Pao, and S.T. Holgate, Characterization of histamine secretion from mechanically dispersed human lung mast cells: effects of anti-IgE, calcium ionophore A23187, compound 48/80, and basic polypeptides. J Immunol, 1982. 129(5): p. 2116-21.

111. Lowman, M.A., et al., Human mast cell heterogeneity: histamine release from mast cells dispersed from skin, lung, adenoids, tonsils, and colon in response to IgE-dependent and nonimmunologic stimuli. J Allergy Clin Immunol, 1988. 81(3): p. 590-7.

112. Tainsh, K.R., et al., Mast cell heterogeneity in man: unique functional properties of skin mast cells in response to a range of polycationic stimuli. Immunopharmacology, 1992. 24(3): p. 171-80.

113. Tomita, U., et al., Direct interactions of mastoparan and compound 48/80 with GTPbinding proteins. J Biochem, 1991. 109(1): p. 184-9.

114. Church, M.K., S. el-Lati, and J.P. Caulfield, Neuropeptide-induced secretion from human skin mast cells. Int Arch Allergy Appl Immunol, 1991. 94(1-4): p. 310-8.

115. Chahdi, A., P.F. Fraundorfer, and M.A. Beaven, Compound 48/80 activates mast cell phospholipase D via heterotrimeric GTP-binding proteins. J Pharmacol Exp Ther, 2000. 292(1): p. 122-30.

116. Kassessinoff, T.A. and F.L. Pearce, Histamine secretion from mast cells stimulated with somatostatin. Agents Actions, 1988. 23(3-4): p. 211-3.

117. Church, M.K. and G.F. Clough, Human skin mast cells: in vitro and in vivo studies. Ann Allergy Asthma Immunol, 1999. 83(5): p. 471-5.

118. Schwartz, L. and T. Huff, Biology of mast cells and basophils., in Allergy: Principles and Practice., E. Middleton, et al., Editors. 1993, Mosby-Year Book, Inc. : St. Louis. p. 135-168. 119. Swindle, E.J. and D.D. Metcalfe, The role of reactive oxygen species and nitric oxide in mast cell-dependent inflammatory processes. Immunol Rev, 2007. 217: p. 186-205.

120. Colgan, J.D. and I.L. Hankel, Signaling pathways critical for allergic airway inflammation. Curr Opin Allergy Clin Immunol, 2010. 10(1): p. 42-7.

121. Beaven, M.A. and R.A. Baumgartner, Downstream signals initiated in mast cells by Fc epsilon RI and other receptors. Curr Opin Immunol, 1996. 8(6): p. 766-72.

122. Kopp, M.V., Omalizumab: Anti-IgE therapy in allergy. Curr Allergy Asthma Rep, 2011. 11(2): p. 101-6.

123. Kuhl, K. and N.A. Hanania, Targeting IgE in asthma. Curr Opin Pulm Med, 2012. 18(1): p. $1-5$.

124. Amin, K., The role of mast cells in allergic inflammation. Respir Med, 2012. 106(1): p.

$9-14$.

125. Ratner, P.H., et al., Use of intranasal cromolyn sodium for allergic rhinitis. Mayo Clin Proc, 2002. 77(4): p. 350-4.

126. Worobec, A.S., Treatment of systemic mast cell disorders. Hematol Oncol Clin North Am, 2000. 14(3): p. 659-87, vii.

127. MacGlashan, D., Jr., Signal mechanisms in the activation of basophils and mast cells. Immunol Ser, 1992. 57: p. 273-99.

128. Dellinger, A., et al., Molecular Interactions of Fullerene Derivatives in Human Serum and Inflammatory Cells. . Inscience: nanotechnology. 1: p. 102-114.

129. Lindstedt, K.A., M.I. Mayranpaa, and P.T. Kovanen, Mast cells in vulnerable atherosclerotic plaques--a view to a kill. J Cell Mol Med, 2007. 11(4): p. 739-58. 
130. Lehtonen-Smeds, E.M., et al., Carotid plaque mast cells associate with atherogenic serum lipids, high grade carotid stenosis and symptomatic carotid artery disease. Results from the helsinki carotid endarterectomy study. Cerebrovasc Dis, 2005. 19(5): p. 291-301.

131. Kovanen, P.T., M. Kaartinen, and T. Paavonen, Infiltrates of activated mast cells at the site of coronary atheromatous erosion or rupture in myocardial infarction. Circulation, 1995. 92(5): p. 1084-8.

132. Ganji, S.H., et al., Niacin inhibits vascular oxidative stress, redox-sensitive genes, and monocyte adhesion to human aortic endothelial cells. Atherosclerosis, 2009. 202(1): p. 68-75.

133. Bot, I., et al., Perivascular mast cells promote atherogenesis and induce plaque destabilization in apolipoprotein E-deficient mice. Circulation, 2007. 115(19): p. 2516-25.

134. Zhao, G., et al., Pivotal role of reactive oxygen species in differential regulation of lipopolysaccharide-induced prostaglandins production in macrophages. Mol Pharmacol, 2013. 83(1): p. 167-78.

135. Buxton, D.B., et al., Report of the National Heart, Lung, and Blood Institute working group on the translation of cardiovascular molecular imaging. Circulation, 2011. 123(19): p. 2157-63.

136. Underhill, H.R., et al., MRI of carotid atherosclerosis: clinical implications and future directions. Nat Rev Cardiol, 2010. 7(3): p. 165-73.

137. Stevenson, S., et al., A stable non-classical metallofullerene family. Nature, 2000. 408(6811): p. 427-8.

138. Nagaraja, T.N., et al., Application of arsenazo III in the preparation and characterization of an albumin-linked, gadolinium-based macromolecular magnetic resonance contrast agent. $\mathrm{J}$ Neurosci Methods, 2006. 157(2): p. 238-45.

139. Bligh, E.G. and W.J. Dyer, A rapid method of total lipid extraction and purification. Can J Biochem Physiol, 1959. 37(8): p. 911-7.

140. Kuzuya, M., et al., Role of lipoprotein-copper complex in copper catalyzed-peroxidation of low-density lipoprotein. Biochim Biophys Acta, 1992. 1123(3): p. 334-41.

141. Koopman, R., G. Schaart, and M.K. Hesselink, Optimisation of oil red O staining permits combination with immunofluorescence and automated quantification of lipids. Histochem Cell Biol, 2001. 116(1): p. 63-8.

142. Rosenfeld, M.E., et al., Progression and disruption of advanced atherosclerotic plaques in murine models. Curr Drug Targets, 2008. 9(3): p. 210-6.

143. Wiant, D., et al., Gamma knife radiosurgery treatment planning for small animals using high-resolution 7 T micro-magnetic resonance imaging. Radiat Res, 2009. 172(5): p. 625-31.

144. Alsaid, H., et al., Biomimetic MRI contrast agent for imaging of inflammation in atherosclerotic plaque of ApoE-/- mice: a pilot study. Invest Radiol, 2009. 44(3): p. 151-8.

145. Moukdar, F., et al., Reduced antioxidant capacity and diet-induced atherosclerosis in uncoupling protein-2-deficient mice. J Lipid Res, 2009. 50(1): p. 59-70.

146. Irani, A.M., et al., Immunohistochemical detection of human basophils in late-phase skin reactions. J Allergy Clin Immunol, 1998. 101(3): p. 354-62.

147. Nicholson, A.C., et al., Role of CD36, the macrophage class B scavenger receptor, in atherosclerosis. Ann N Y Acad Sci, 2001. 947: p. 224-8.

148. Brown, M.S., et al., The scavenger cell pathway for lipoprotein degradation: specificity of the binding site that mediates the uptake of negatively-charged LDL by macrophages. $\mathrm{J}$ Supramol Struct, 1980. 13(1): p. 67-81. 
149. Collins, R.F., et al., Uptake of oxidized low density lipoprotein by CD36 occurs by an actin-dependent pathway distinct from macropinocytosis. J Biol Chem, 2009. 284(44): p. 3028897.

150. Rahaman, S.O., et al., A CD36-dependent signaling cascade is necessary for macrophage foam cell formation. Cell Metab, 2006. 4(3): p. 211-21.

151. Partovi, S., et al., Contrast-enhanced ultrasound for assessing carotid atherosclerotic plaque lesions. AJR Am J Roentgenol, 2012. 198(1): p. W13-9.

152. Voros, S., et al., Coronary atherosclerosis imaging by coronary CT angiography: current status, correlation with intravascular interrogation and meta-analysis. JACC Cardiovasc Imaging, 2011. 4(5): p. 537-48.

153. Amirbekian, V., et al., Detecting and assessing macrophages in vivo to evaluate atherosclerosis noninvasively using molecular MRI. Proc Natl Acad Sci U S A, 2007. 104(3): p. 961-6.

154. Kahn, E., et al., Analysis of CD36 expression on human monocytic cells and atherosclerotic tissue sections with quantum dots: investigation by flow cytometry and spectral imaging microscopy. Anal Quant Cytol Histol, 2006. 28(1): p. 14-26.

155. Canet-Soulas, E. and D. Letourneur, Biomarkers of atherosclerosis and the potential of MRI for the diagnosis of vulnerable plaque. MAGMA, 2007. 20(3): p. 129-42.

156. Davies, J.R., et al., Radionuclide imaging for the detection of inflammation in vulnerable plaques. J Am Coll Cardiol, 2006. 47(8 Suppl): p. C57-68.

157. Hyafil, F., et al., Noninvasive detection of macrophages using a nanoparticulate contrast agent for computed tomography. Nat Med, 2007. 13(5): p. 636-41.

158. Kooi, M.E., et al., Accumulation of ultrasmall superparamagnetic particles of iron oxide in human atherosclerotic plaques can be detected by in vivo magnetic resonance imaging. Circulation, 2003. 107(19): p. 2453-8.

159. Lipinski, M.J., et al., MRI to detect atherosclerosis with gadolinium-containing immunomicelles targeting the macrophage scavenger receptor. Magn Reson Med, 2006. 56(3): p. 601-10.

160. Lipinski, M.J., et al., Macrophage-specific lipid-based nanoparticles improve cardiac magnetic resonance detection and characterization of human atherosclerosis. JACC Cardiovasc Imaging, 2009. 2(5): p. 637-47.

161. Nahrendorf, M., et al., Noninvasive vascular cell adhesion molecule-1 imaging identifies inflammatory activation of cells in atherosclerosis. Circulation, 2006. 114(14): p. 1504-11.

162. Nahrendorf, M., D.E. Sosnovik, and R. Weissleder, MR-optical imaging of cardiovascular molecular targets. Basic Res Cardiol, 2008. 103(2): p. 87-94.

163. Rudd, J.H., et al., Imaging atherosclerotic plaque inflammation with [18F]fluorodeoxyglucose positron emission tomography. Circulation, 2002. 105(23): p. 2708-11.

164. Uchida, M., et al., Protein cage nanoparticles bearing the LyP-1 peptide for enhanced imaging of macrophage-rich vascular lesions. ACS Nano, 2011. 5(4): p. 2493-502.

165. Endemann, G., et al., CD36 is a receptor for oxidized low density lipoprotein. J Biol Chem, 1993. 268(16): p. 11811-6.

166. Fatouros, P.P., et al., In vitro and in vivo imaging studies of a new endohedral metallofullerene nanoparticle. Radiology, 2006. 240(3): p. 756-64.

167. Kolosnjaj, J., H. Szwarc, and F. Moussa, Toxicity studies of fullerenes and derivatives. Adv Exp Med Biol, 2007. 620: p. 168-80. 
168. Fillmore, H.L., et al., Conjugation of functionalized gadolinium metallofullerenes with IL-13 peptides for targeting and imaging glial tumors. Nanomedicine (Lond), 2011. 6(3): p. 44958.

169. Shu, C., et al., Facile preparation of a new gadofullerene-based magnetic resonance imaging contrast agent with high $1 \mathrm{H}$ relaxivity. Bioconjug Chem, 2009. 20(6): p. 1186-93.

170. Zhang, J., et al., High relaxivity trimetallic nitride (Gd3N) metallofullerene MRI contrast agents with optimized functionality. Bioconjug Chem, 2010. 21(4): p. 610-5.

171. Aschberger, K., et al., Review of fullerene toxicity and exposure--appraisal of a human health risk assessment, based on open literature. Regul Toxicol Pharmacol, 2010. 58(3): p. 45573.

172. Clark, A.R., Anti-inflammatory functions of glucocorticoid-induced genes. Mol Cell Endocrinol, 2007. 275(1-2): p. 79-97.

173. Geetha, N., et al., Signal integration and coincidence detection in the mitogen-activated protein kinaselextracellular signal-regulated kinase (ERK) cascade: concomitant activation of receptor tyrosine kinases and of LRP-1 leads to sustained ERK phosphorylation via downregulation of dual specificity phosphatases (DUSP1 and -6). J Biol Chem, 2011. 286(29): p. 25663-74.

174. Huang, C.Y. and T.H. Tan, DUSPs, to MAP kinases and beyond. Cell Biosci, 2012. 2(1): p. 24.

175. Jin, Y., et al., Dual-specificity phosphatase 1 as a pharmacogenetic modifier of inhaled steroid response among asthmatic patients. J Allergy Clin Immunol, 2010. 126(3): p. 618-25 e12.

176. Joanny, E., et al., Anti-inflammatory effects of selective glucocorticoid receptor modulators are partially dependent on up-regulation of dual specificity phosphatase $1 . \mathrm{Br} \mathrm{J}$ Pharmacol, 2012. 165(4b): p. 1124-36.

177. Abraham, S.M. and A.R. Clark, Dual-specificity phosphatase 1: a critical regulator of innate immune responses. Biochem Soc Trans, 2006. 34(Pt 6): p. 1018-23.

178. Dickinson, R.J. and S.M. Keyse, Diverse physiological functions for dual-specificity MAP kinase phosphatases. J Cell Sci, 2006. 119(Pt 22): p. 4607-15.

179. Farooq, A. and M.M. Zhou, Structure and regulation of MAPK phosphatases. Cell Signal, 2004. 16(7): p. 769-79.

180. Keyse, S.M., An emerging family of dual specificity MAP kinase phosphatases. Biochim Biophys Acta, 1995. 1265(2-3): p. 152-60.

181. Abraham, S.M., et al., Antiinflammatory effects of dexamethasone are partly dependent on induction of dual specificity phosphatase 1. J Exp Med, 2006. 203(8): p. 1883-9.

182. Zhang, Y.L. and C. Dong, MAP kinases in immune responses. Cell Mol Immunol, 2005. 2(1): p. 20-7.

183. Zhao, Q., et al., The role of mitogen-activated protein kinase phosphatase-1 in the response of alveolar macrophages to lipopolysaccharide: attenuation of proinflammatory cytokine biosynthesis via feedback control of p38. J Biol Chem, 2005. 280(9): p. 8101-8.

184. Black, J.L., B.G. Oliver, and M. Roth, Molecular mechanisms of combination therapy with inhaled corticosteroids and long-acting beta-agonists. Chest, 2009. 136(4): p. 1095-100.

185. Johnson, S.R., An assay to evaluate the long term effects of inflammatory mediators on airway smooth muscle: evidence that TNF(alpha)up-regulates 5-HT(2A) mediated contraction. Br J Pharmacol, 2002. 137(7): p. 943-4. 
186. Chong, L.K., et al., Salmeterol inhibition of mediator release from human lung mast cells by beta-adrenoceptor-dependent and independent mechanisms. Br J Pharmacol, 1998. 123(5): p. 1009-15.

187. Maier, J.V., et al., Dual specificity phosphatase 1 knockout mice show enhanced susceptibility to anaphylaxis but are sensitive to glucocorticoids. Mol Endocrinol, 2007. 21(11): p. 2663-71.

188. Zhou, Z. and P.A. Magriotis, A new method for the functionalization of [60] fullerene: an unusual 1,3-dipolar cycloaddition pathway leading to a C60 housane derivative. Org Lett, 2005. 7(26): p. 5849-51.

189. Zhou, Z., D.I. Schuster, and S.R. Wilson, Selective syntheses of novel polyether fullerene multiple adducts. J Org Chem, 2003. 68(20): p. 7612-7.

190. Claxson, A., et al., The anti-inflammatory effects of D-myo-inositol-1.2.6-trisphosphate (PP56) on animal models of inflammation. Agents Actions, 1990. 29(1-2): p. 68-70.

191. Kepley, C.L. and N. Cohen, Evidence for human mast cell nonreleaser phenotype. J Allergy Clin Immunol, 2003. 112(2): p. 457-9.

192. Zhou, Z., et al., A novel class of compounds with cutaneous wound healing properties. J Biomed Nanotechnol, 2010. 6(5): p. 605-11.

193. Irani, A.M., et al., Detection of MCT and MCTC types of human mast cells by immunohistochemistry using new monoclonal anti-tryptase and anti-chymase antibodies. $\mathrm{J}$ Histochem Cytochem, 1989. 37(10): p. 1509-15.

194. Zhao, W., et al., Fc gamma RIIa, not Fc gamma RIIb, is constitutively and functionally expressed on skin-derived human mast cells. J Immunol, 2006. 177(1): p. 694-701.

195. Lin, Y.W. and J.L. Yang, Cooperation of ERK and SCFSkp2 for MKP-1 destruction provides a positive feedback regulation of proliferating signaling. J Biol Chem, 2006. 281(2): p. 915-26.

196. Cates, C.J., et al., Regular treatment with salmeterol and inhaled steroids for chronic asthma: serious adverse events. Cochrane Database Syst Rev, 2013. 3: p. CD006922.

197. Schacke, H., W.D. Docke, and K. Asadullah, Mechanisms involved in the side effects of glucocorticoids. Pharmacol Ther, 2002. 96(1): p. 23-43.

198. Adiseshaiah, P., et al., A novel gadolinium-based trimetasphere metallofullerene for application as a magnetic resonance imaging contrast agent. Invest Radiol, 2013. 48(11): p. 74554.

199. Dellinger, A., et al., Functionalization of gadolinium metallofullerenes for detecting atherosclerotic plaque lesions by cardiovascular magnetic resonance. J Cardiovasc Magn Reson, 2013. 15: p. 7.

200. Kassel, O., et al., Glucocorticoids inhibit MAP kinase via increased expression and decreased degradation of MKP-1. EMBO J, 2001. 20(24): p. 7108-16.

201. Shang, F. and A. Taylor, Ubiquitin-proteasome pathway and cellular responses to oxidative stress. Free Radic Biol Med, 2011. 51(1): p. 5-16.

202. Davies, K.J., Oxidative stress: the paradox of aerobic life. Biochem Soc Symp, 1995. 61: p. 1-31.

203. Park, H., et al., Lighting the fires within: the cell biology of autoinflammatory diseases. Nat Rev Immunol, 2012. 12(8): p. 570-80.

204. Arab, H.H. and M.M. El-Sawalhi, Carvedilol alleviates adjuvant-induced arthritis and subcutaneous air pouch edema: modulation of oxidative stress and inflammatory mediators. Toxicol Appl Pharmacol, 2013. 268(2): p. 241-8. 
205. Drafi, F., et al., Pharmacological influence on processes of adjuvant arthritis: Effect of the combination of an antioxidant active substance with methotrexate. Interdiscip Toxicol, 2012. 5(2): p. 84-91.

206. Lee, E.Y., et al., Alpha-lipoic acid suppresses the development of collagen-induced arthritis and protects against bone destruction in mice. Rheumatol Int, 2007. 27(3): p. 225-33.

207. Park, M.K., et al., Retinal attenuates inflammatory arthritis by reciprocal regulation of IL-17-producing T cells and Foxp3(+) regulatory $T$ cells and the inhibition of osteoclastogenesis. Immunol Lett, 2012. 148(1): p. 59-68.

208. Zhang, W. and S.M. Dai, Mechanisms involved in the therapeutic effects of Paeonia lactiflora Pallas in rheumatoid arthritis. Int Immunopharmacol, 2012. 14(1): p. 27-31.

209. Haqqi, T.M., et al., Prevention of collagen-induced arthritis in mice by a polyphenolic fraction from green tea. Proc Natl Acad Sci U S A, 1999. 96(8): p. 4524-9.

210. Skoldstam, L., L. Hagfors, and G. Johansson, An experimental study of a Mediterranean diet intervention for patients with rheumatoid arthritis. Ann Rheum Dis, 2003. 62(3): p. 208-14.

211. Jaswal, S., et al., Antioxidant status in rheumatoid arthritis and role of antioxidant therapy. Clin Chim Acta, 2003. 338(1-2): p. 123-9.

212. Yudoh, K., et al., Water-soluble C60 fullerene prevents degeneration of articular cartilage in osteoarthritis via down-regulation of chondrocyte catabolic activity and inhibition of cartilage degeneration during disease development. Arthritis Rheum, 2007. 56(10): p. 3307-18.

213. Castor, C.W., The microscopic structure of normal human synovial tissue. Arthritis Rheum, 1960. 3: p. 140-51.

214. Nigrovic, P.A. and D.M. Lee, Mast cells in inflammatory arthritis. Arthritis Res Ther, 2005. 7(1): p. 1-11.

215. Gruber, B., D. Ballan, and P.D. Gorevic, IgE rheumatoid factors: quantification in synovial fluid and ability to induce synovial mast cell histamine release. Clin Exp Immunol, 1988. 71(2): p. 289-94.

216. Gruber, B., et al., Characterization and functional studies of rheumatoid synovial mast cells. Activation by secretagogues, anti-IgE, and a histamine-releasing lymphokine. Arthritis Rheum, 1986. 29(8): p. 944-55.

217. Huber, L.C., et al., Synovial fibroblasts: key players in rheumatoid arthritis. Rheumatology (Oxford), 2006. 45(6): p. 669-75.

218. Noss, E.H. and M.B. Brenner, The role and therapeutic implications of fibroblast-like synoviocytes in inflammation and cartilage erosion in rheumatoid arthritis. Immunol Rev, 2008. 223: p. 252-70.

219. Choi, Y., J.R. Arron, and M.J. Townsend, Promising bone-related therapeutic targets for rheumatoid arthritis. Nat Rev Rheumatol, 2009. 5(10): p. 543-8.

220. Bai, X.C., et al., Reactive oxygen species stimulates receptor activator of NF-kappaB ligand expression in osteoblast. J Biol Chem, 2005. 280(17): p. 17497-506.

221. Garrett, I.R., et al., Oxygen-derived free radicals stimulate osteoclastic bone resorption in rodent bone in vitro and in vivo. J Clin Invest, 1990. 85(3): p. 632-9.

222. Kamata, H. and H. Hirata, Redox regulation of cellular signalling. Cell Signal, 1999. 11(1): p. 1-14.

223. Lean, J.M., et al., A crucial role for thiol antioxidants in estrogen-deficiency bone loss. J Clin Invest, 2003. 112(6): p. 915-23.

224. Malbec, O. and M. Daeron, The mast cell IgG receptors and their roles in tissue inflammation. Immunol Rev, 2007. 217: p. 206-21. 
225. Avnet, S., et al., Interferon-alpha inhibits in vitro osteoclast differentiation and renal cell carcinoma-induced angiogenesis. Int J Oncol, 2007. 30(2): p. 469-76.

226. Reers, M., et al., Mitochondrial membrane potential monitored by JC-1 dye. Methods Enzymol, 1995. 260: p. 406-17.

227. Smiley, S.T., et al., Intracellular heterogeneity in mitochondrial membrane potentials revealed by a J-aggregate-forming lipophilic cation JC-1. Proc Natl Acad Sci U S A, 1991. 88(9): p. 3671-5.

228. Cossarizza, A., et al., A new method for the cytofluorimetric analysis of mitochondrial membrane potential using the J-aggregate forming lipophilic cation 5,5',6,6'-tetrachloro1,1',3,3'-tetraethylbenzimidazolcarbocyanine iodide (JC-1). Biochem Biophys Res Commun, 1993. 197(1): p. 40-5.

229. Wruck, C.J., et al., Role of oxidative stress in rheumatoid arthritis: insights from the Nrf2-knockout mice. Ann Rheum Dis, 2011. 70(5): p. 844-50.

230. Feyerabend, T.B., et al., Cre-mediated cell ablation contests mast cell contribution in models of antibody-and T cell-mediated autoimmunity. Immunity, 2011. 35(5): p. 832-44.

231. Holmdahl, R., et al., The molecular pathogenesis of collagen-induced arthritis in mice--a model for rheumatoid arthritis. Ageing Res Rev, 2002. 1(1): p. 135-47.

232. Brand, D.D., K.A. Latham, and E.F. Rosloniec, Collagen-induced arthritis. Nat Protoc, 2007. 2(5): p. 1269-75.

233. Nigrovic, P.A., et al., C5a receptor enables participation of mast cells in immune complex arthritis independently of Fcgamma receptor modulation. Arthritis Rheum, 2010. 62(11): p. 3322-33.

234. Dellinger, A., et al., Effects of novel nanomaterials on allergic mediator release from human mast cells and basophils through non-Ige mediated pathways. J Nanomed Nanotechnol, 2012. 3(153).

235. Zorov, D.B., M. Juhaszova, and S.J. Sollott, Mitochondrial ROS-induced ROS release: an update and review. Biochim Biophys Acta, 2006. 1757(5-6): p. 509-17.

236. Morgan, M.J. and Z.G. Liu, Crosstalk of reactive oxygen species and NF-kappaB signaling. Cell Res, 2011. 21(1): p. 103-15.

237. Sun, S.C., The noncanonical NF-kappaB pathway. Immunol Rev, 2012. 246(1): p. 12540.

238. Steinitz, K., A simple bedside test for renal function; the semiquantitative determination of blood creatinine. Harefuah, 1947. 32(10): p. 174.

239. Eklund, K.K., Mast cells in the pathogenesis of rheumatic diseases and as potential targets for anti-rheumatic therapy. Immunol Rev, 2007. 217: p. 38-52.

240. Niedermeier, M., T. Pap, and A. Korb, Therapeutic opportunities in fibroblasts in inflammatory arthritis. Best Pract Res Clin Rheumatol, 2010. 24(4): p. 527-40.

241. Solomon, S., D. Kassahn, and H. Illges, The role of the complement and the Fc gamma $R$ system in the pathogenesis of arthritis. Arthritis Res Ther, 2005. 7(4): p. 129-35.

242. Phillips, D.C., et al., Aberrant reactive oxygen and nitrogen species generation in rheumatoid arthritis (RA): causes and consequences for immune function, cell survival, and therapeutic intervention. Antioxid Redox Signal, 2010. 12(6): p. 743-85.

243. Foley, S., et al., Cellular localisation of a water-soluble fullerene derivative. Biochem Biophys Res Commun, 2002. 294(1): p. 116-9.

244. Porter, A.E., et al., Uptake of C60 by human monocyte macrophages, its localization and implications for toxicity: studied by high resolution electron microscopy and electron tomography. Acta Biomater, 2006. 2(4): p. 409-19. 
245. Bamborough, P., M.A. Morse, and K.P. Ray, Targeting IKKbeta for the treatment of rheumatoid arthritis. Drug News Perspect, 2010. 23(8): p. 483-90.

246. Marcu, K.B., et al., NF-kappaB signaling: multiple angles to target OA. Curr Drug Targets, 2010. 11(5): p. 599-613.

247. Roman-Blas, J.A. and S.A. Jimenez, Targeting NF-kappaB: a promising molecular therapy in inflammatory arthritis. Int Rev Immunol, 2008. 27(5): p. 351-74.

248. Yamamoto, Y. and R.B. Gaynor, Therapeutic potential of inhibition of the NF-kappaB pathway in the treatment of inflammation and cancer. J Clin Invest, 2001. 107(2): p. 135-42.

249. Handel, M.L., L.Q. Nguyen, and T.P. Lehmann, Inhibition of transcription factors by anti-inflammatory and anti-rheumatic drugs: can variability in response be overcome? Clin Exp Pharmacol Physiol, 2000. 27(3): p. 139-44.

250. Majumdar, S. and B.B. Aggarwal, Methotrexate suppresses NF-kappaB activation through inhibition of IkappaBalpha phosphorylation and degradation. J Immunol, 2001. 167(5): p. 2911-20.

251. Ibrahim, M., et al., Fullerene derivative as anti-HIV protease inhibitor: molecular modeling and QSAR approaches. Mini Rev Med Chem, 2012. 12(6): p. 447-51.

252. Luo, Z., et al., Development of calixarenes, cyclodextrins and fullerenes as new platforms for anti-HIV drug design: an overview. Mini Rev Med Chem, 2013. 13(8): p. 1160-5.

253. Kornev, A.B., et al., Synthesis and antiviral activity of highly water-soluble polycarboxylic derivatives of [70]fullerene. Chem Commun (Camb), 2011. 47(29): p. 8298-300.

254. Zhu, Z., D.I. Schuster, and M.E. Tuckerman, Molecular dynamics study of the connection between flap closing and binding of fullerene-based inhibitors of the HIV-1 protease. Biochemistry, 2003. 42(5): p. 1326-33.

255. Marcorin, G.L., et al., Design and synthesis of novel [60]fullerene derivatives as potential HIV aspartic protease inhibitors. Org Lett, 2000. 2(25): p. 3955-8.

256. Rancan, F., et al., Cytotoxicity and photocytotoxicity of a dendritic C(60) mono-adduct and a malonic acid C(60) tris-adduct on Jurkat cells. J Photochem Photobiol B, 2002. 67(3): p. 157-62.

257. Liu, Y. and H. Wang, Nanomedicine: Nanotechnology tackles tumours. Nat Nanotechnol, 2007. 2(1): p. 20-1.

258. Saathoff, J.G., et al., In vitro toxicity assessment of three hydroxylated fullerenes in human skin cells. Toxicol In Vitro, 2011. 25(8): p. 2105-12. 


\section{APPENDIX A}

\section{DESIGN SYNTHESIS OF ATCA}
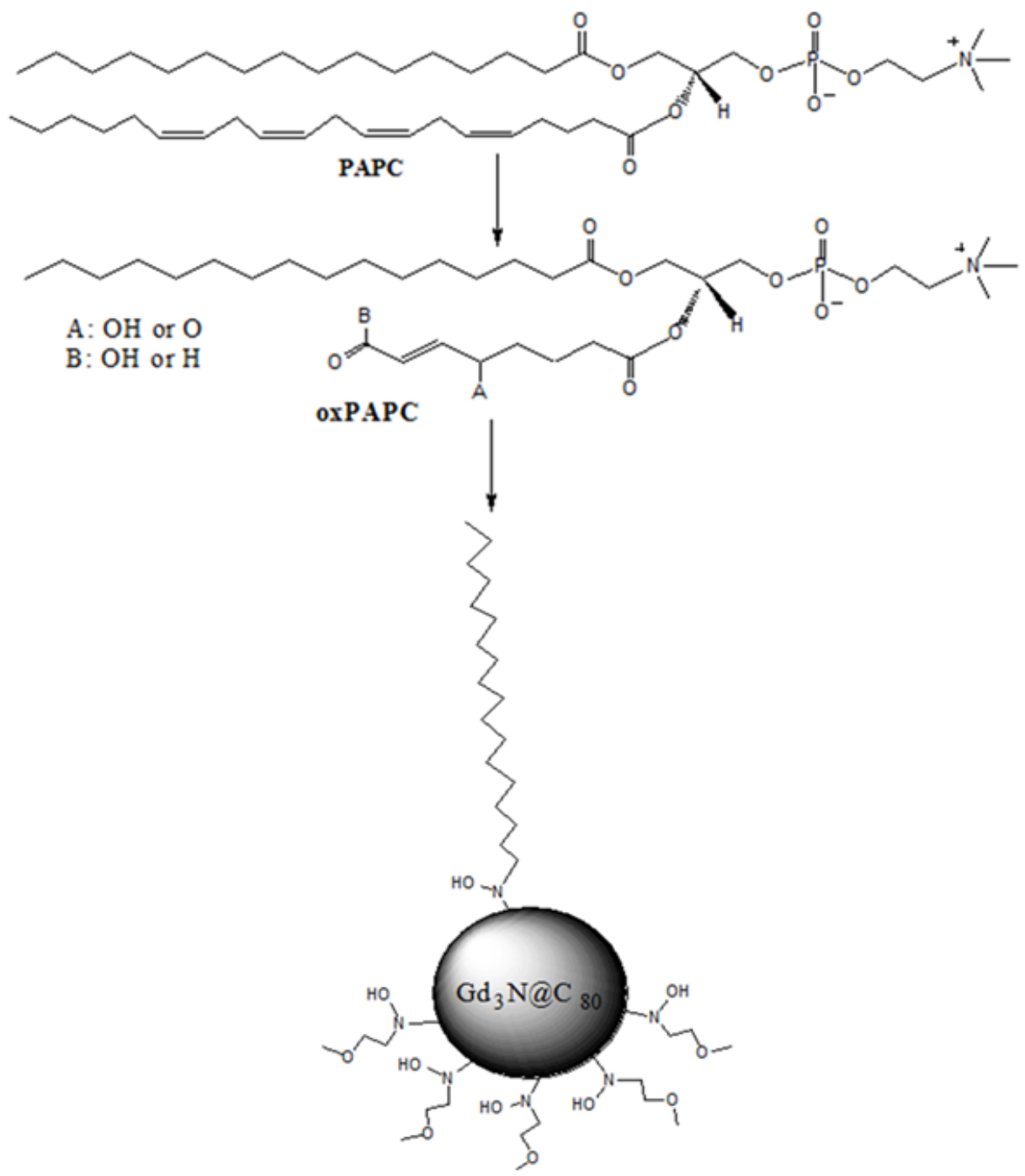

ATCA

Figure A.1. Design Synthesis of ATCA.

Top: PAPC and its oxidation product oxPAPC as CD36 ligands under myeloperoxidase (MPO) $-\mathrm{H}_{2} \mathrm{O}_{2}-\mathrm{NO}_{2}$ - system. Bottom: structure of the ATCA TMS 
derivative used in this study. To develop the CD36 ligands, PAPC (1-palmitoyl-2arachidonyl-sn-glycero-3-phosphocholine or 1-hexadecanoyl-2-eicosatetra-5', $8^{\prime}, 11^{\prime}, 14^{\prime}$ enoyl-sn-glycero-3-phosphocholine) was oxidized by the myeloperoxidase (MPO)- $\mathrm{H}_{2} \mathrm{O}_{2}$ $\mathrm{NO}_{2}$ - system to generate oxidized PAPC (oxPAPC) which includes HOdiA-PC, KOdiAPC, HOOA-PC and KOOA-PC species. Briefly, $1 \mathrm{mg} / \mathrm{ml}$ PAPC solution of small unilamellar vesicles was prepared and oxidized by a mixture of $30 \mathrm{nM} \mathrm{MPO}, 100 \mu \mathrm{M}$ glucose and $100 \mathrm{ng} / \mathrm{ml}$ glucose oxidase (generating $\mathrm{H}_{2} \mathrm{O}_{2}$ ), and $0.5 \mathrm{mM} \mathrm{NaNO}_{2}$ for $24 \mathrm{~h}$ at $37^{\circ} \mathrm{C}$. The reaction was stopped by adding butylated hydroxyltoluene (BHT) and catalase. The oxPAPC lipids were extracted from the oxidized PAPC vesicles with chloroform three times. The combined organic phases were evaporated under nitrogen to dryness. Liquid chromatography was used to demonstrate the successful oxidation of PAPC and to quantify the ratio of oxidized lipids to those non-oxidized. The bioactive and oxidized PAPC lipids were used in the preparation of TMS-encapsulated liposomes.

The final ATCA was made by mixing 20 parts of regular phosphocholine lipids (DPPC), one part of oxPAPC and five parts of amphiphilic TMS, having one $\mathrm{C}_{18}$ aliphatic chain and five short PEG polar groups] in chloroform under nitrogen and the mixture was evaporated to dryness under vacuum to form a thin film on the flask wall. The material was hydrated by sonicating the film materials in buffered saline ( $\mathrm{pH} 7.4)$ using a bath sonicator under nitrogen. The crude liposomes were extruded three times with $400 \mathrm{~nm}, 200 \mathrm{~nm}$, and $100 \mathrm{~nm}$ nucleopore membranes each to produce the final ATCA sample as a brownish suspension. The control sample has the same ratio of both amphiphilic TMS derivative and DPPC as ATCA (approximately 30,000-32,000 
TMS/liposome), but do not contain any CD36 ligands. The incorporation of colored TMS derivatives in liposome bilayers was further confirmed by buoyant density test, where the functionalized TMS stayed associated with lipid bilayers on the top of a $40 \%$ sucrose cushion under high speed centrifugation conditions that typically precipitate any TMS materials if not tightly associated with lipid membranes. ATCA samples can be further purified by eluting them on a size exclusion Sephadex column to remove any lipids unincorporated in the liposome bilayer. The co-elution of lipids (DPPC and oxPAPC) with TMS derivatives further demonstrated their tight association in the bilayer structure. 


\section{APPENDIX B}

\section{DLS, ZETA POTENTIAL, AND RELAXIVITY OF LIPOSOME CONTROL AND ATCA}
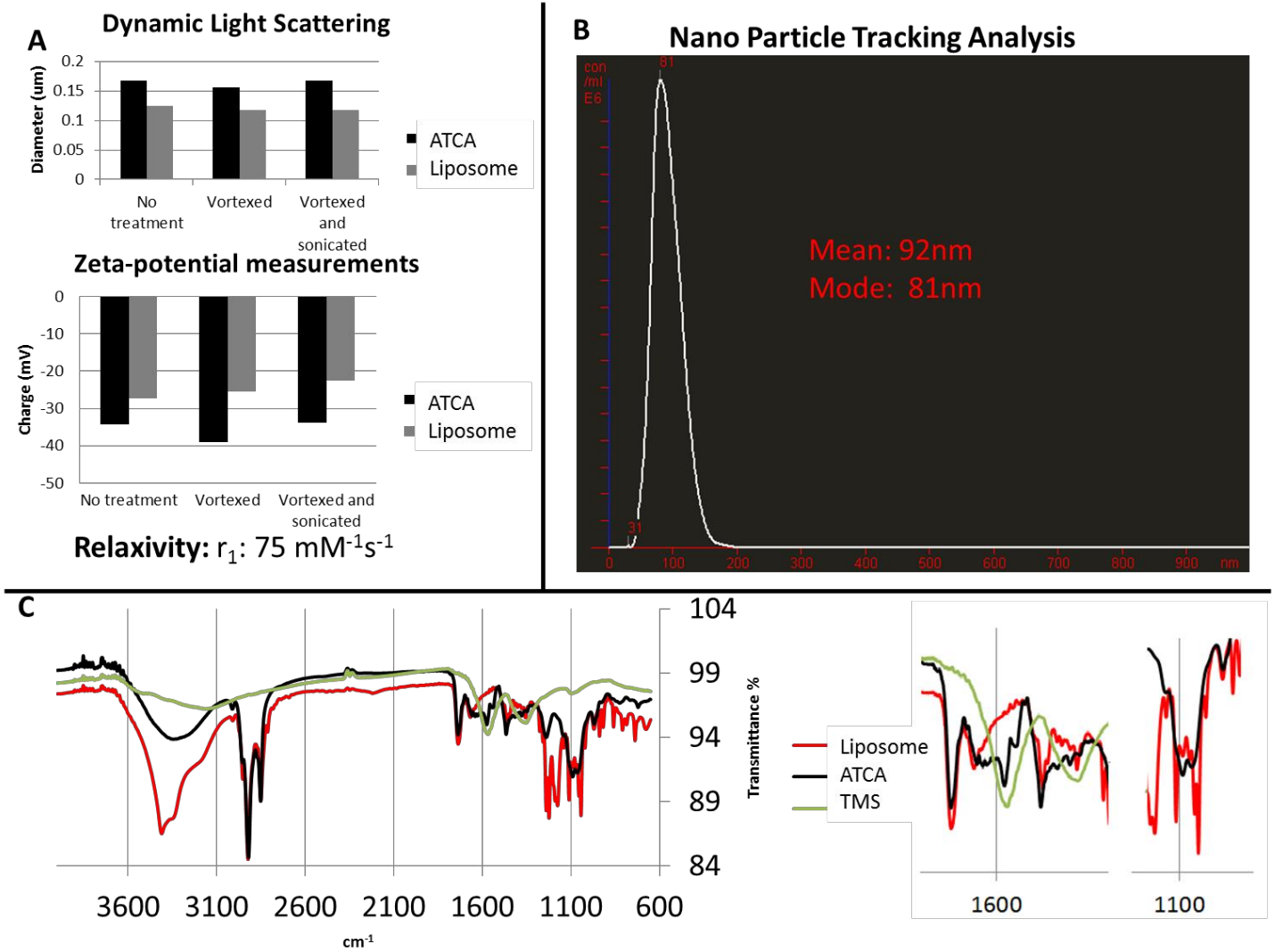

Figure B.1. DLS, Zeta Potential, and Relaxivity of Liposome Control and ATCA.

A. ATCA (black) and liposome control (grey) were diluted 1:100,000 and either subjected directly for analysis, vortexed, or sonicated and vortexed to reduce agglomeration. Dynamic light scattering (DLS) and zeta potential values (Zetatrac, Microtrac Instrument) were used to assess the physical characteristics of the nanoparticle conjugates in solution. The size distribution profile of ATCA and control (no CD36 
ligands) are $0.1565 \mu \mathrm{m}$ and $0.1172 \mu \mathrm{m}$, respectively while the zeta potential was 39.02 and $-25.50 \mathrm{mV}$ suggesting that the ATCA are extremely stable. The size distribution and zeta potential did not significantly change after vigorous vortexing or sonication. The relaxivity r1 of ATCA was calculated by a benchtop relaxometer and determined to be $75 \mathrm{mM}-1 \mathrm{~s}-1$ B. Nanosight nanoparticle tracking of ATCA. ATCA was diluted as above and particle size distribution was measured (106 particles/ml) for 90 seconds. The Nanosight nanoparticle tracking analysis instrument was used and revealed a mean particle size of $0.092 \mu \mathrm{m}$. The smaller size distribution for the Nanosight is a result of individualized particle distribution of size, whereas DLS results are volume based distribution of size. Volume based distribution results in larger particle size due to the fact that larger particles have higher volumes and thus heavily influences mean measurements. C. FTIR analysis of ATCA. Fourier transform infrared spectroscopy (FTIR) spectrum of TMS (green), Liposome control (red), and ATCA (black). The presence of TMS inside the liposome was verified by the following peaks at $1093(\mathrm{C}-\mathrm{N})$, $1361(\mathrm{~N}-\mathrm{O}), 1571(\mathrm{~N}-\mathrm{O})$, and $3149(\mathrm{O}-\mathrm{H}) \mathrm{cm}-1$. In the ATCA sample the peak at 1361 cm-1 is broadened by the $\mathrm{C}-\mathrm{N}$ bond. The $\mathrm{N}-\mathrm{O}$ peak broadens the peak around $1400 \mathrm{~cm}-1$. The $1571 \mathrm{~cm}-1$ peak shows that a new bond type is present that did not exist in the liposome. The O-H bond of the TMS slightly shifts the alcohol absorption peak to the right and broadens its appearance. Next, the presence of PAPC conjugated to the liposome was proven by the peaks at 1090 (PO2-) and 823 (P-O-C) as previously described. In the case the P-O-C bond the sharp peak at $823 \mathrm{~cm}-1$ was broadened and for the PO2- a shoulder was formed at $1090 \mathrm{~cm}-1$. 